\title{
Cooperative Bond Activation Reactions with Nickel and Palladium Carbene Complexes with a $\mathrm{PC}_{\text {CarbeneS }} \mathrm{S}$ pincer ligand
}

\section{Supporting Information}

Lennart T. Scharf, Alexander Kowsari, Kai-Stephan Feichtner, Thorsten Scherpf and Viktoria H. Gessner*

Lehrstuhl für Anorganische Chemie II, Ruhr-Universität Bochum, 44780 Bochum, Germany

1. NMR Spectra ....................................................................................................................... 2

2. Crystal structure determination .................................................................... 18

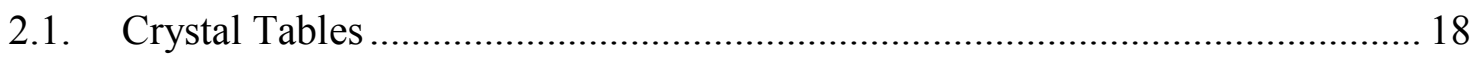

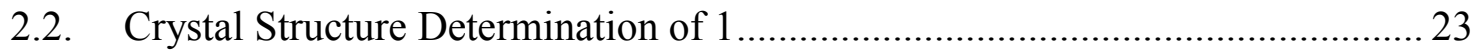

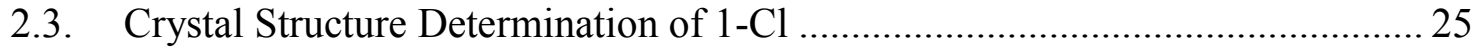

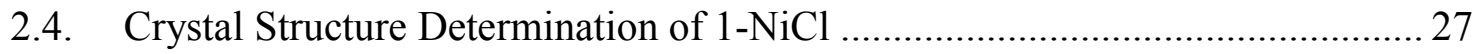

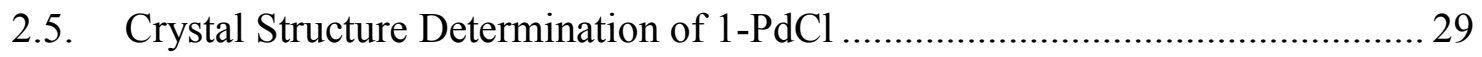

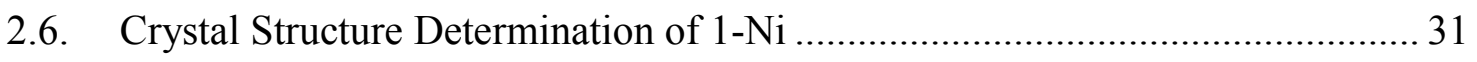

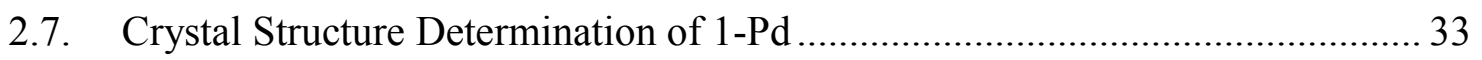

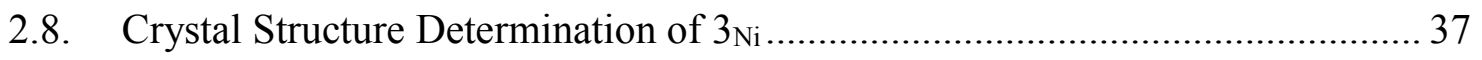

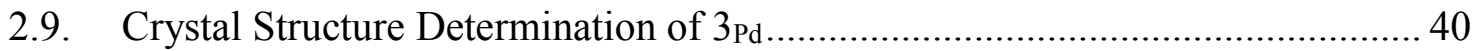

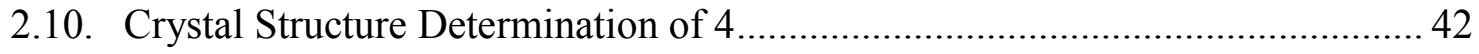

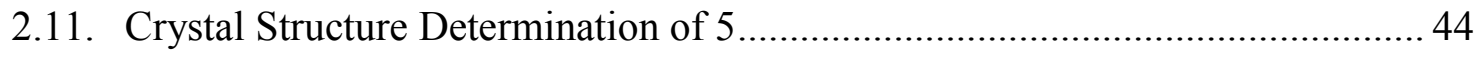

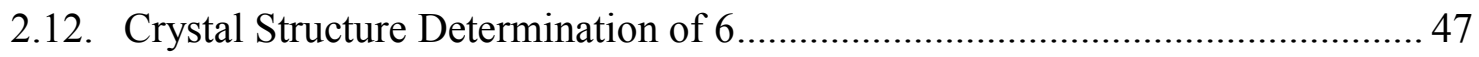

3. Computational Studies ............................................................................................ 51 


\section{NMR Spectra}

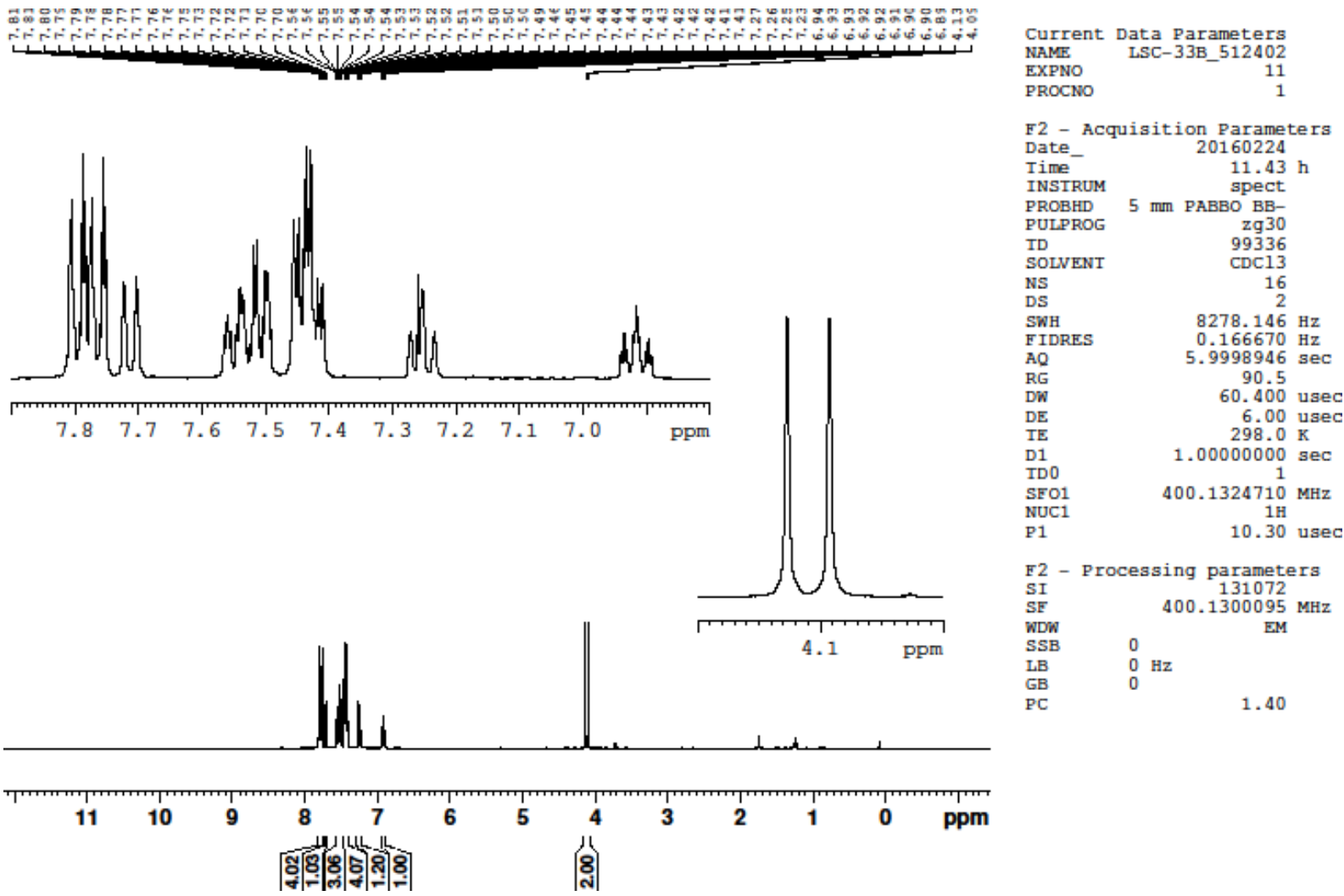

Figure S1. ${ }^{1} \mathrm{H}$ NMR spectrum of $\mathbf{2}$ (unlabelled peaks between 0 and $2 \mathrm{ppm}$ belong to residual diethyl ether, water and grease).

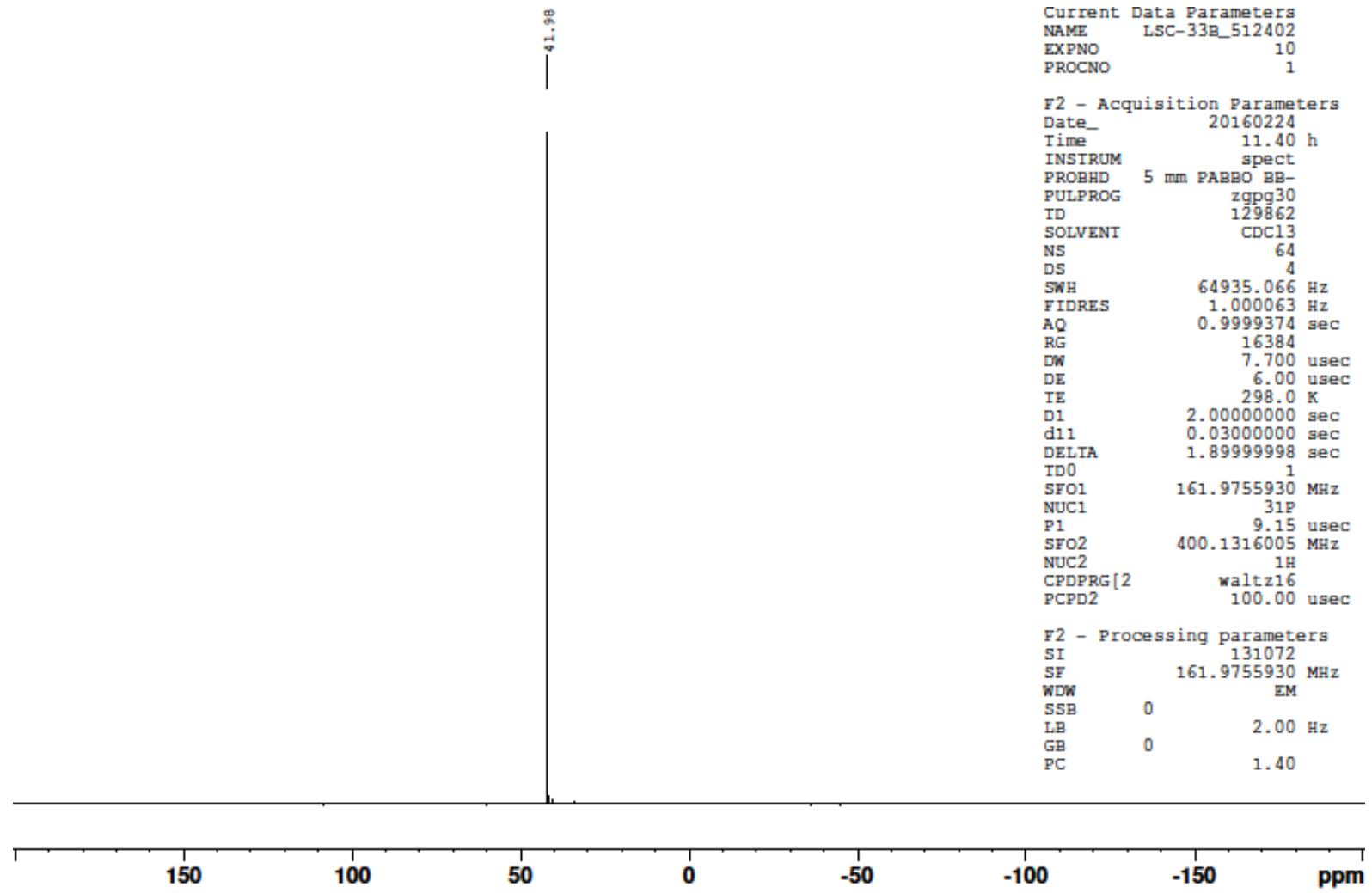

Figure S2. ${ }^{31} \mathrm{P}$ NMR spectrum of 2 . 

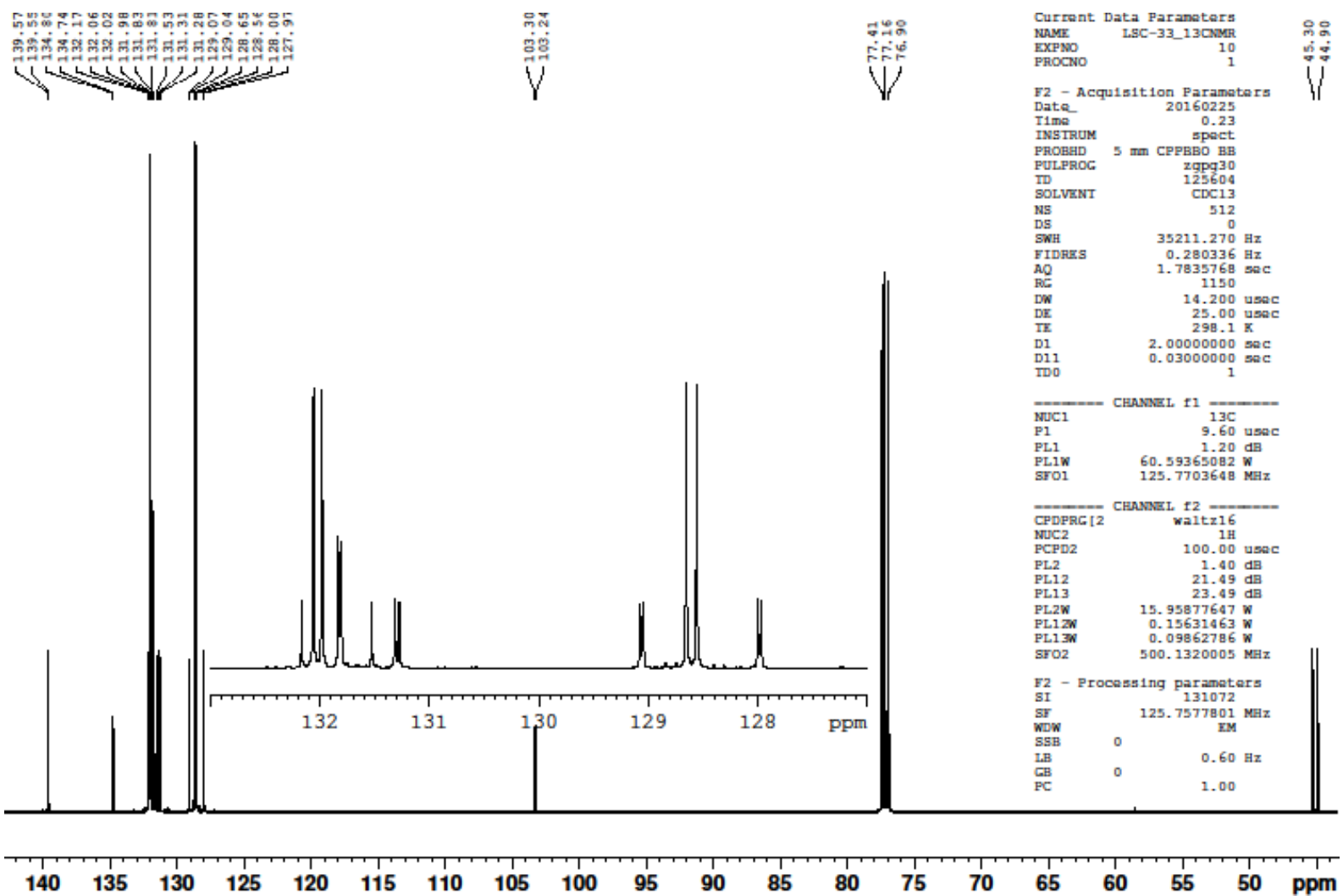

Figure S3. $\left\{{ }^{1} \mathrm{H}\right\}{ }^{13} \mathrm{C}$ NMR spectrum of 2 .
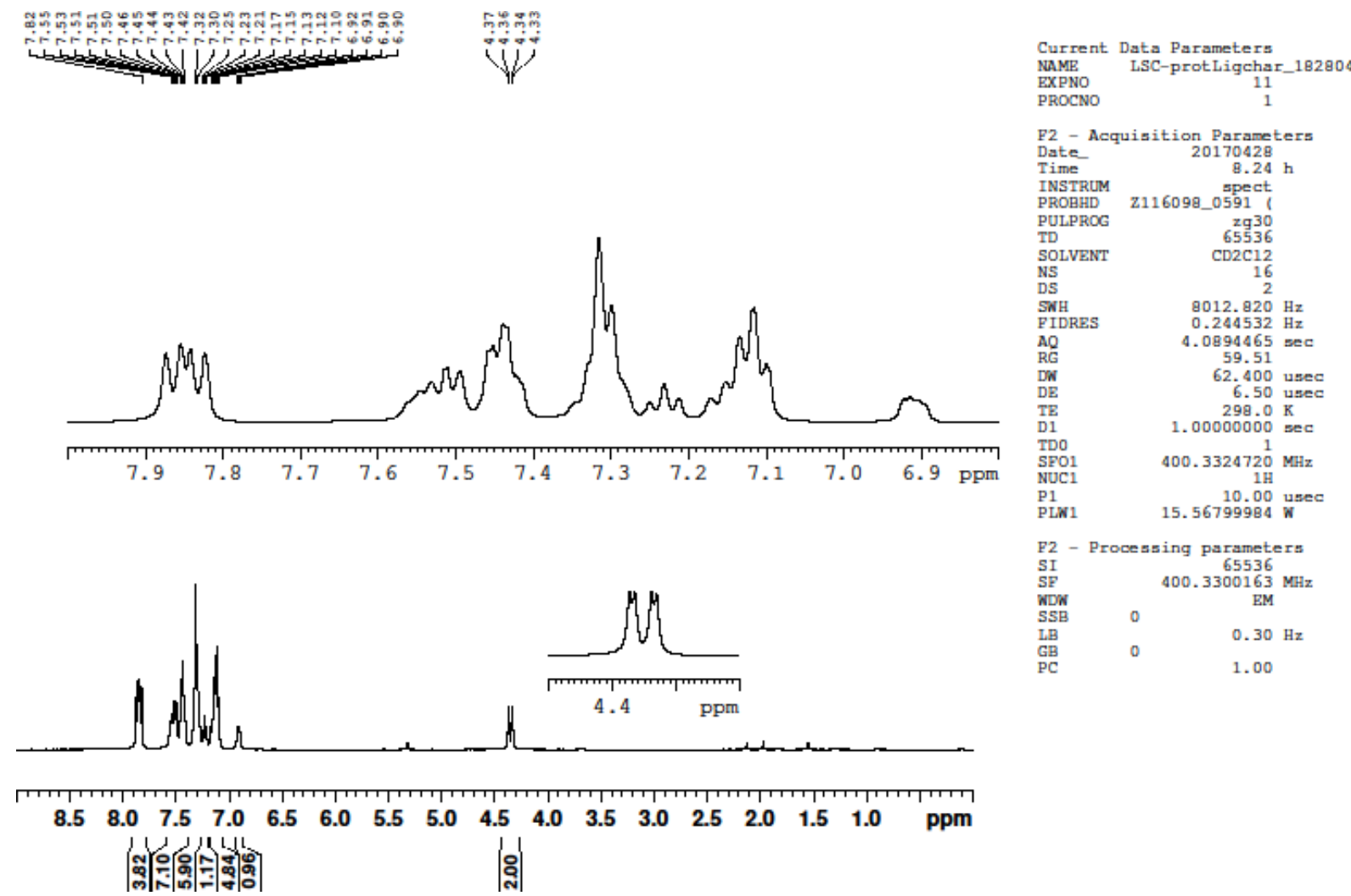

Figure S4. ${ }^{1} \mathrm{H}$ NMR spectrum of $\mathbf{1}$ (unlabelled peaks between 0 and $2.5 \mathrm{ppm}$ belong to residual water, acetone and acetonitrile). 


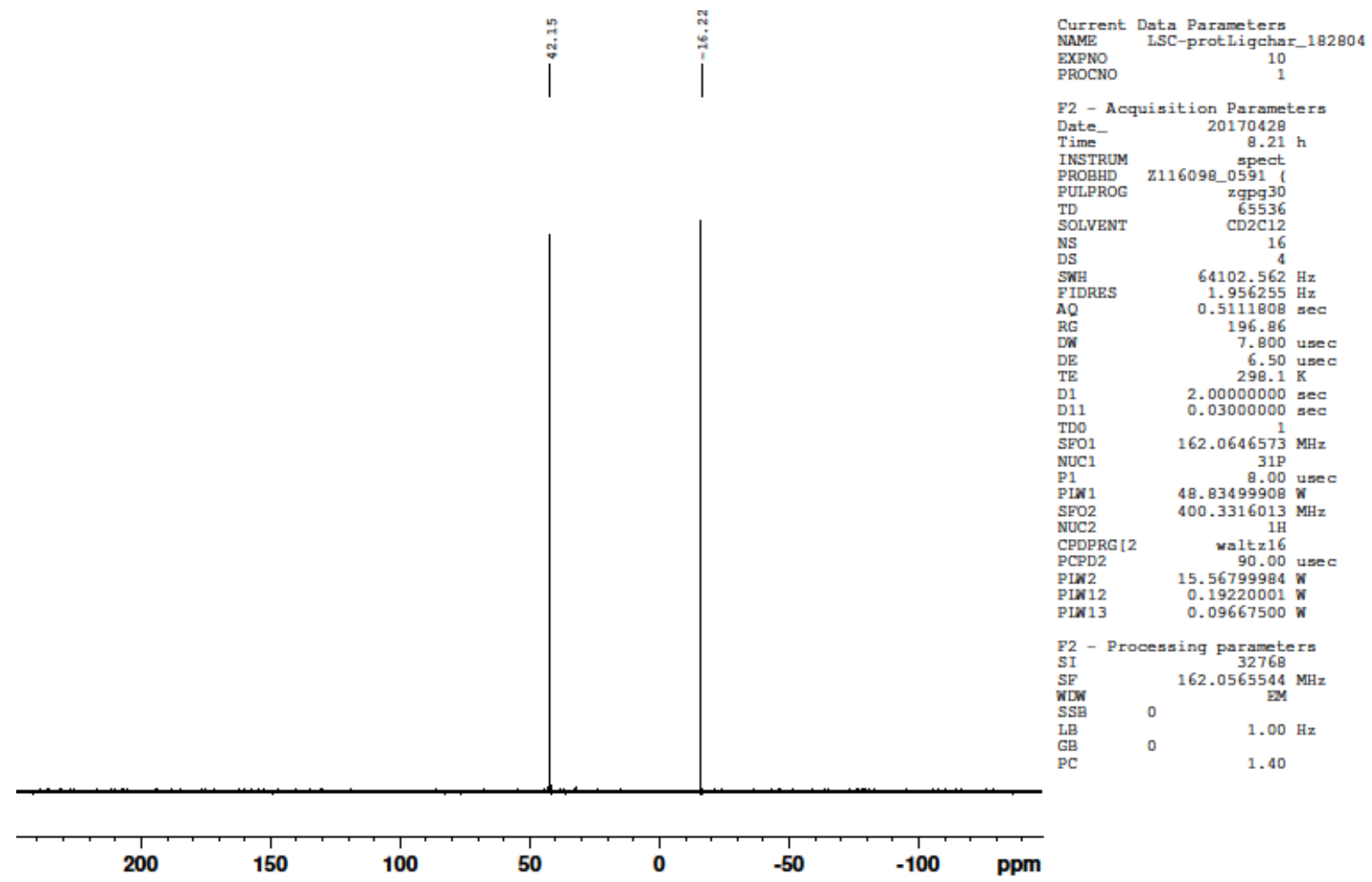

Figure S5. ${ }^{31} \mathrm{P}$ NMR spectrum of $\mathbf{1}$.

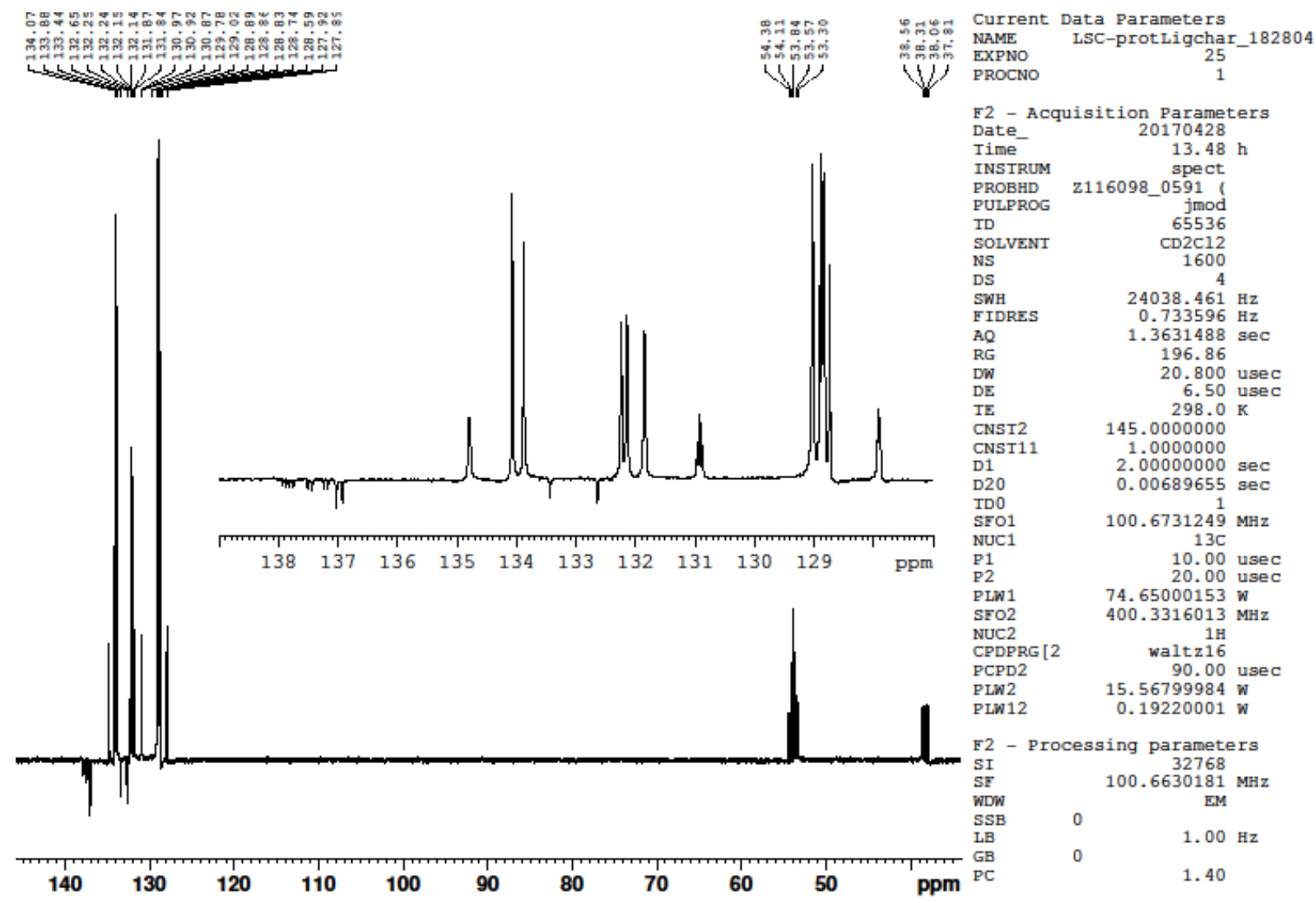

Figure S6. $\left\{{ }^{1} \mathrm{H}\right\}{ }^{13} \mathrm{C}$ APT NMR spectrum of $\mathbf{1}$. 


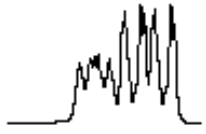

8. $2 \mathrm{ppm}$
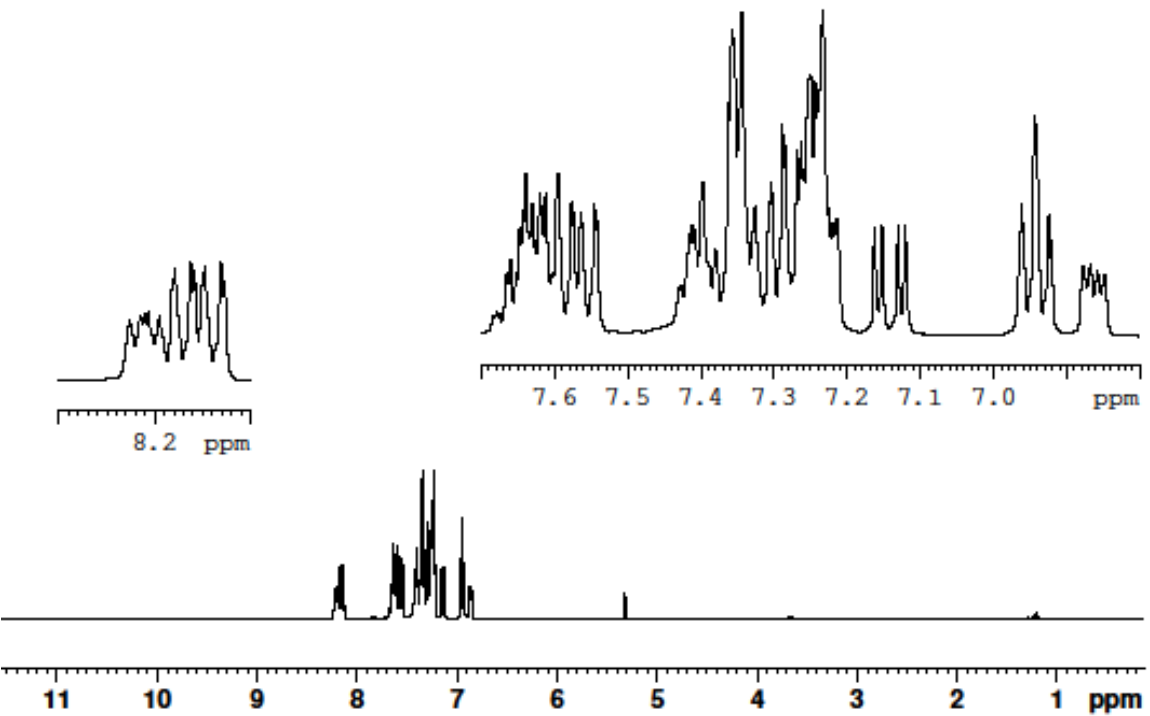

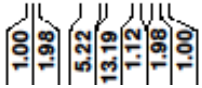

Figure S7. ${ }^{1} \mathrm{H}$ NMR spectrum of 1-Cl (unlabelled peaks between 1 and 4 ppm belong to residual pentane and diethyl ether).

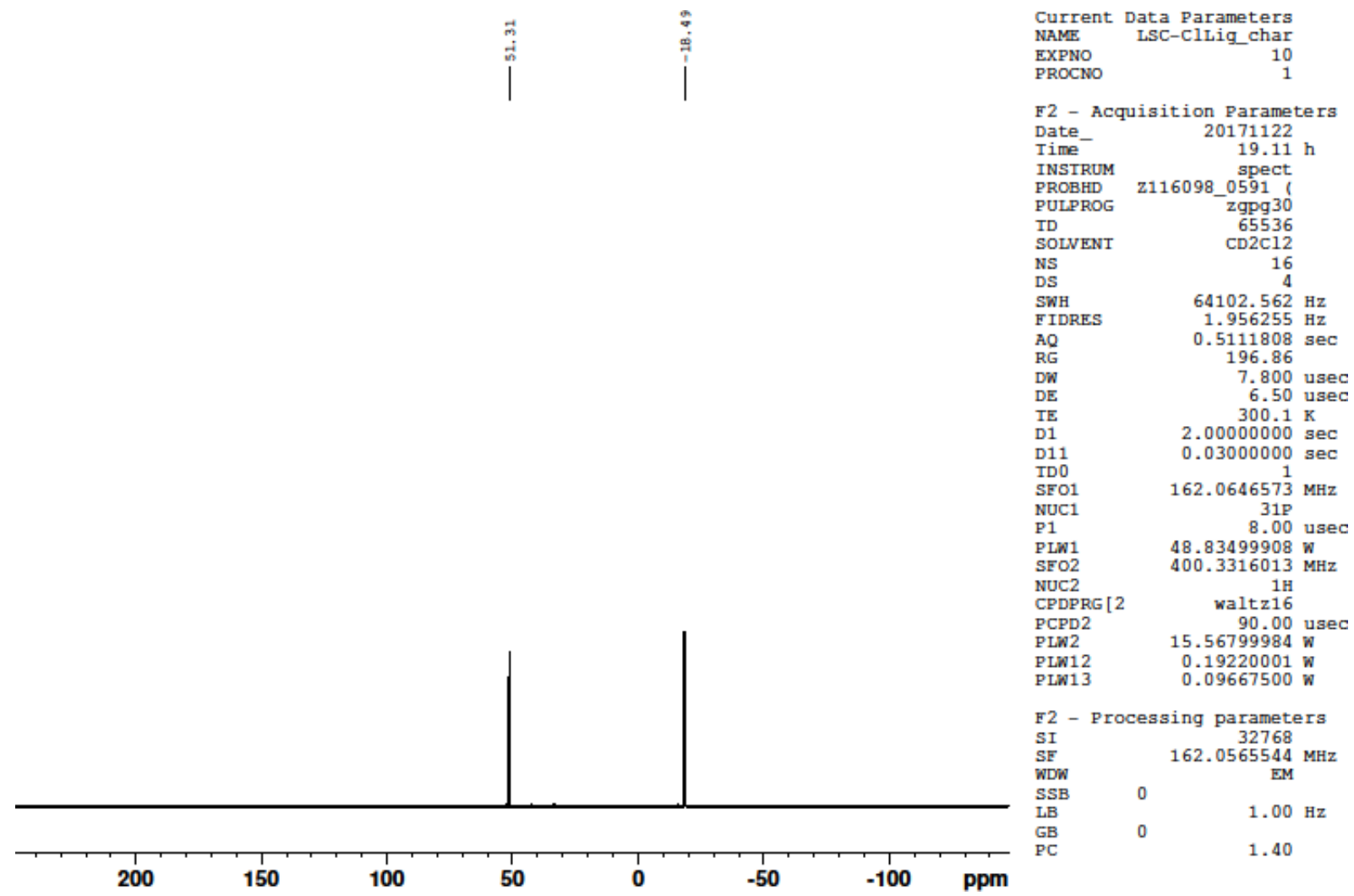

Figure S8. ${ }^{31} \mathrm{P}$ NMR spectrum of 1-Cl. 


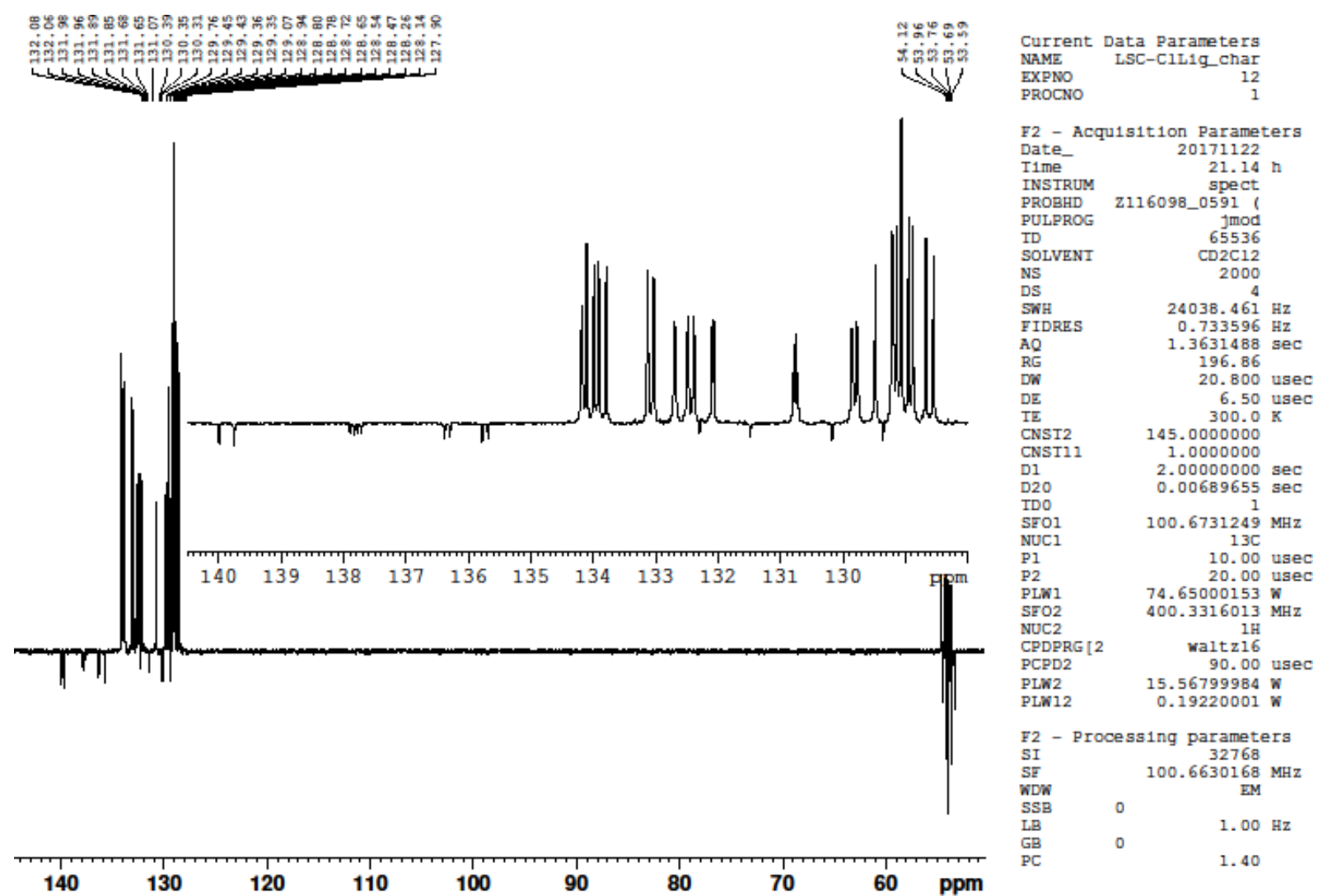

Figure S9. $\left\{{ }^{1} \mathrm{H}\right\}{ }^{13} \mathrm{C}$ APT NMR spectrum of 1-Cl.

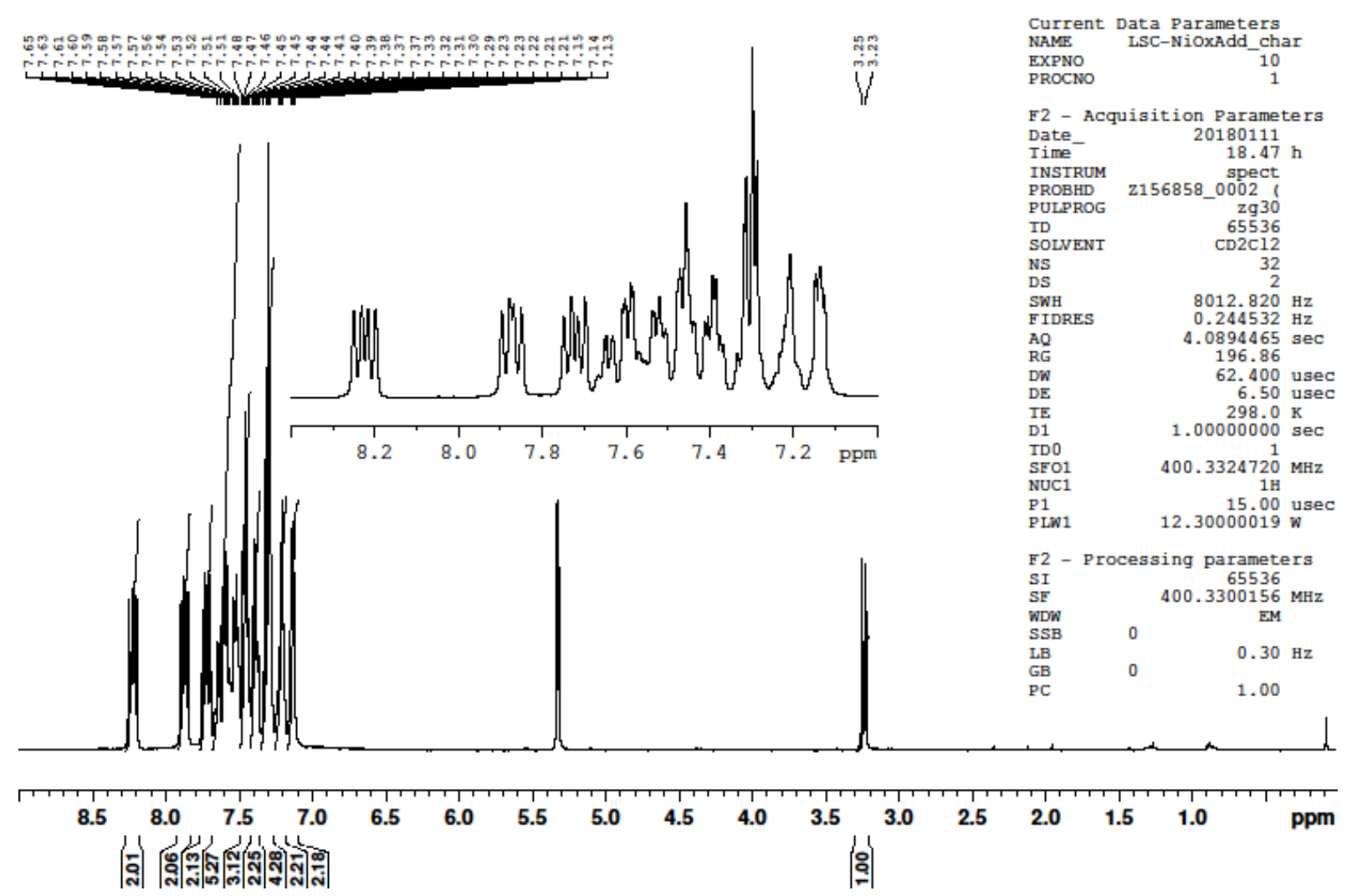

Figure S10. ${ }^{1} \mathrm{H}$ NMR spectrum of $\mathbf{1 - N i C l}$ (unlabelled peaks between 0 and $1.5 \mathrm{ppm}$ belong to residual pentane and grease). 


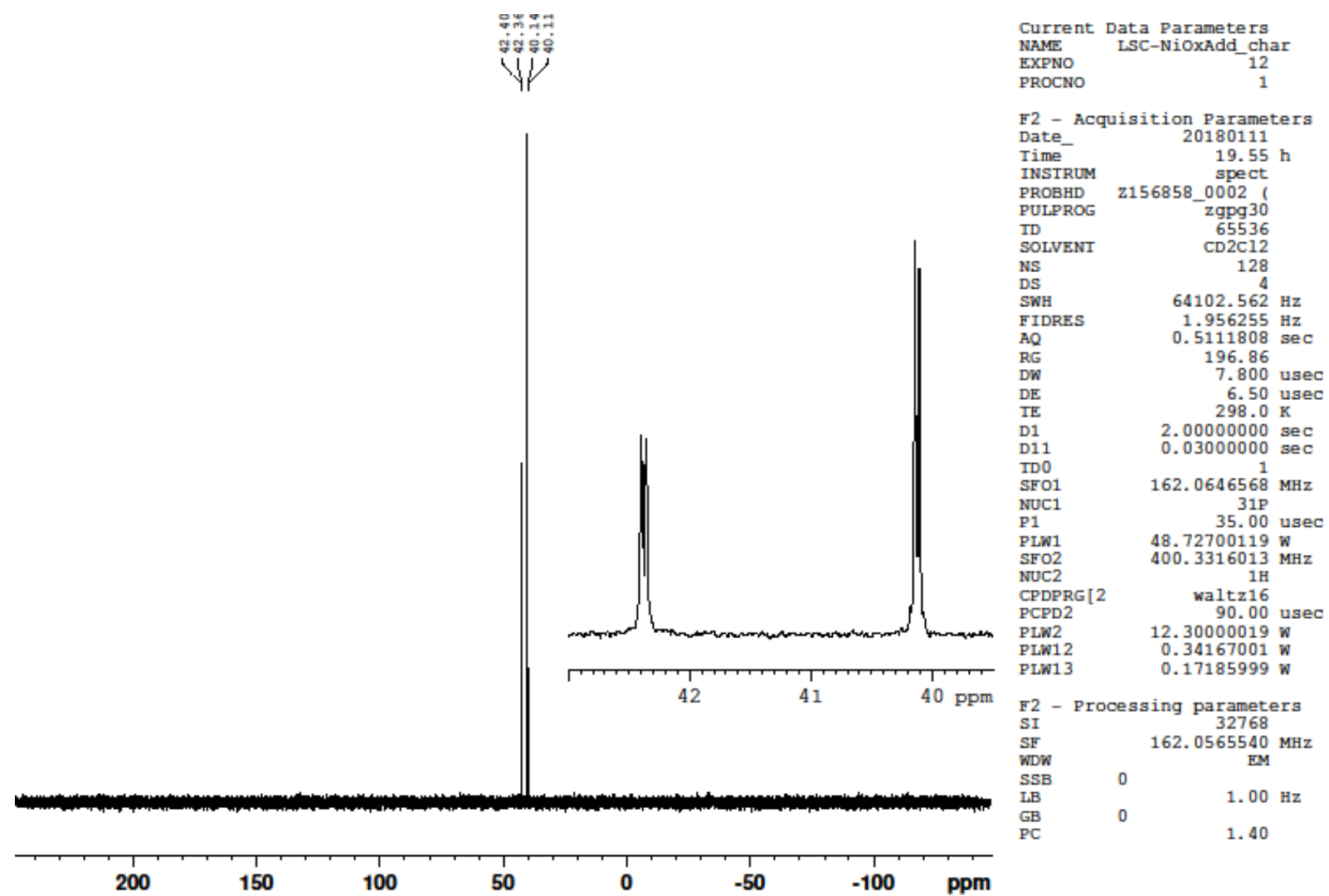

Figure S11. ${ }^{31} \mathrm{P}$ NMR spectrum of 1-NiCl.
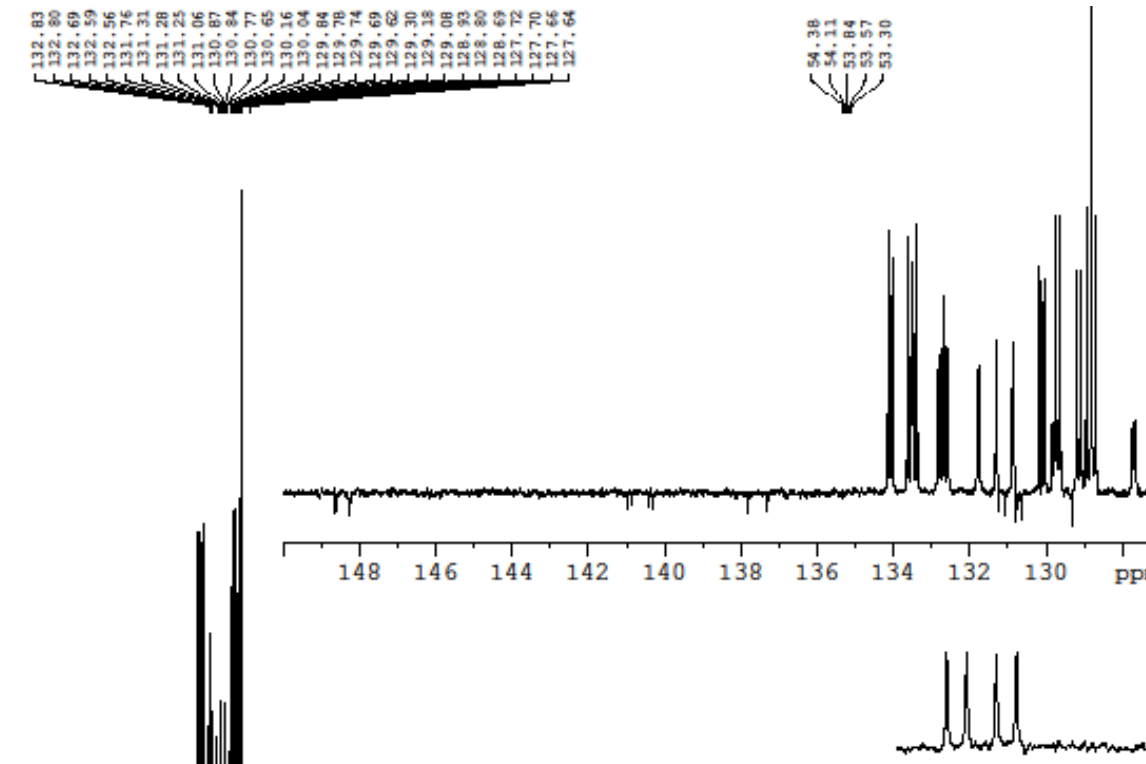

৪ะ유 Current Data Parameters 격ㄹ NAME LSC-N10xAdd_cha (y) EXPNO

F2 - Acqu1s1tion Parameters Date_ 20180111 IIme 23.43 Z156858 spect PULPROG SOLVENT

NS

FIDRES $\quad 24038.461 \mathrm{~Hz}$

AQ $1.3631488 \mathrm{sec}$

196.86

20.800 usec

$298.0 \mathrm{~K}$
$\mathrm{C}$

CNST2 145.0000000

2.0000000

$\begin{array}{ll}\text { D1 } & 2.00000000 \mathrm{sec} \\ \text { D20 } & 0.00689655 \mathrm{sec}\end{array}$ IDO $\quad 1$ 1 SFO1 $\quad 100.6731249$

P1 10.00 usec P2 20.00 usec PLW 1 46.68000031 W SFO2 400.3316013 MH NUC2 2 1 . $\begin{array}{lr}\text { CPDPRG [2 } & \text { waltz16 } \\ \text { PCPD2 } & 90.00 \text { usec }\end{array}$ PLW2 12.30000019 w $\begin{array}{lr}\text { PLW2 } & 12.30000019 \\ \text { PLW12 } & 0.34167001\end{array}$

F2 - Processing parameters $\begin{array}{lc}\text { SI } & 32768 \\ \text { SF } & 100.6630177 \mathrm{MHz} \\ \text { WDW } & \text { EM }\end{array}$ LBB $\quad 1.00 \mathrm{~Hz}$

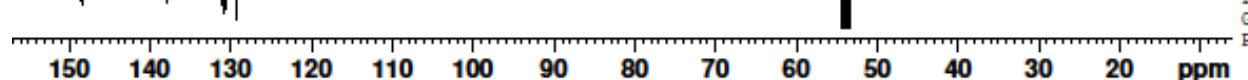

1.40

Figure S12. $\left\{{ }^{1} \mathrm{H}\right\}{ }^{13} \mathrm{C}$ APT NMR spectrum of 1-NiCl. 


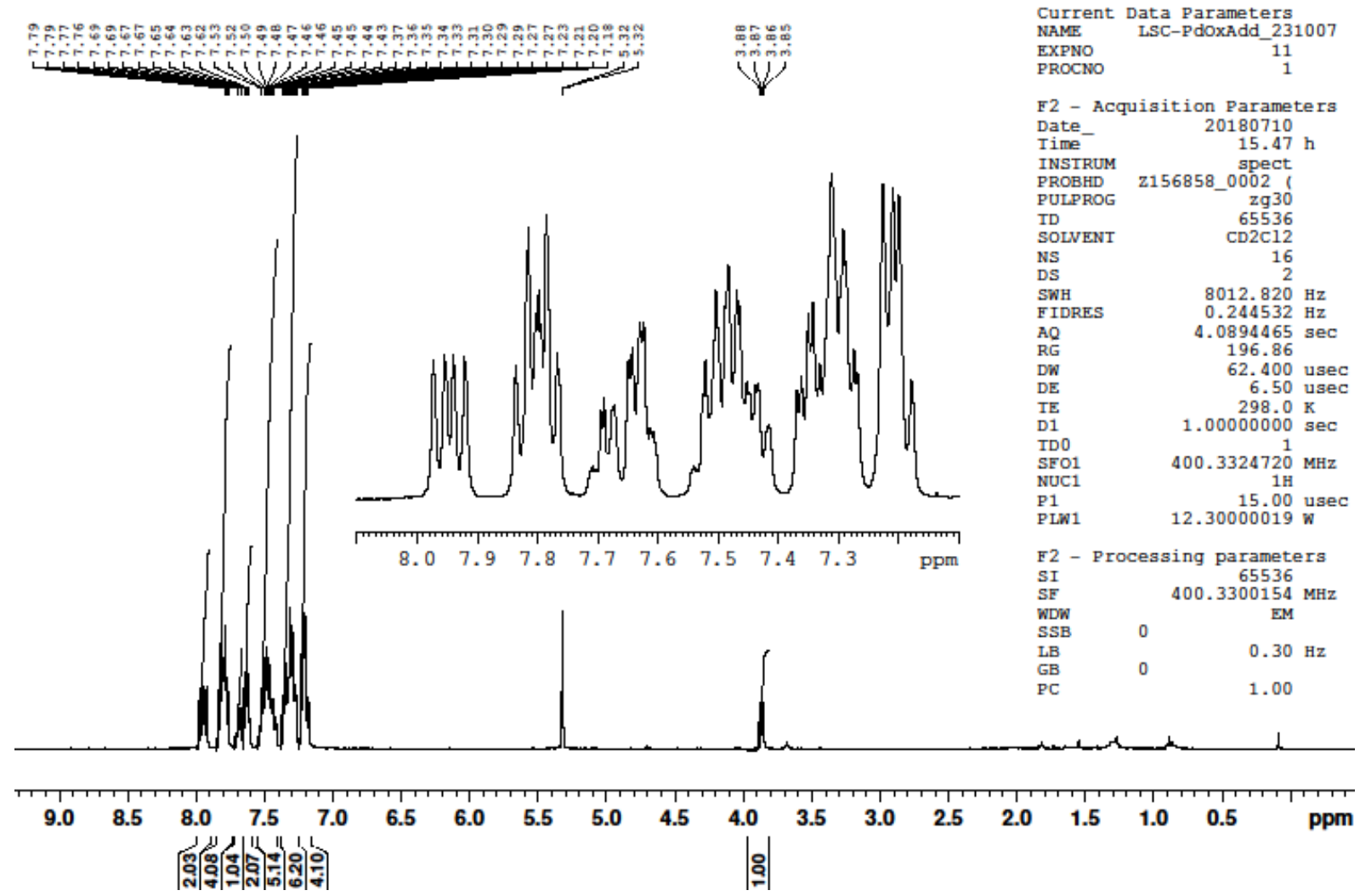

Figure S13. ${ }^{1} \mathrm{H}$ NMR spectrum of 1-PdCl (unlabelled peaks between 0 and $3.7 \mathrm{ppm}$ belong to residual pentane and THF).

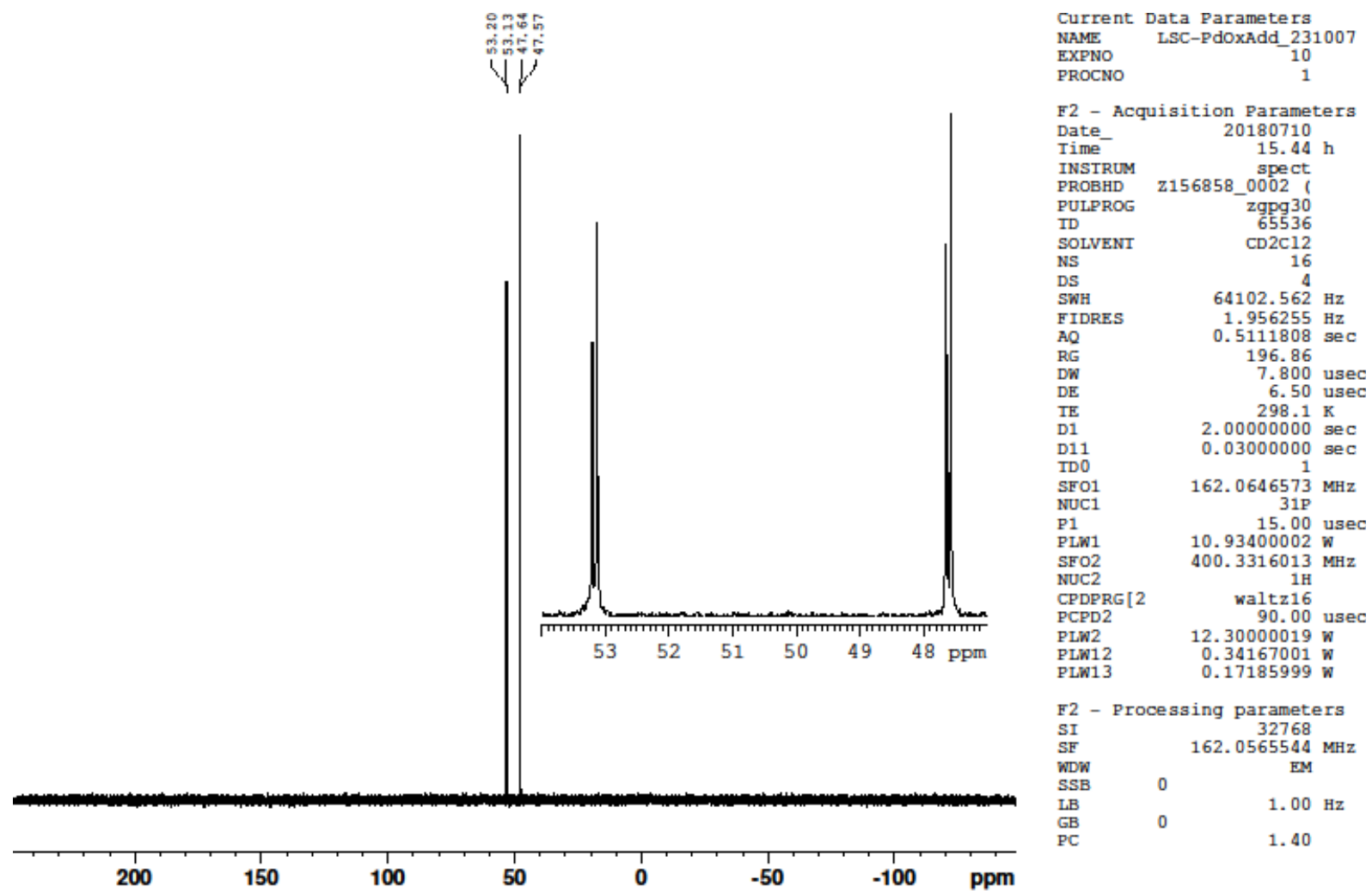

Figure S14. ${ }^{31} \mathrm{P}$ NMR spectrum of 1-PdCl. 


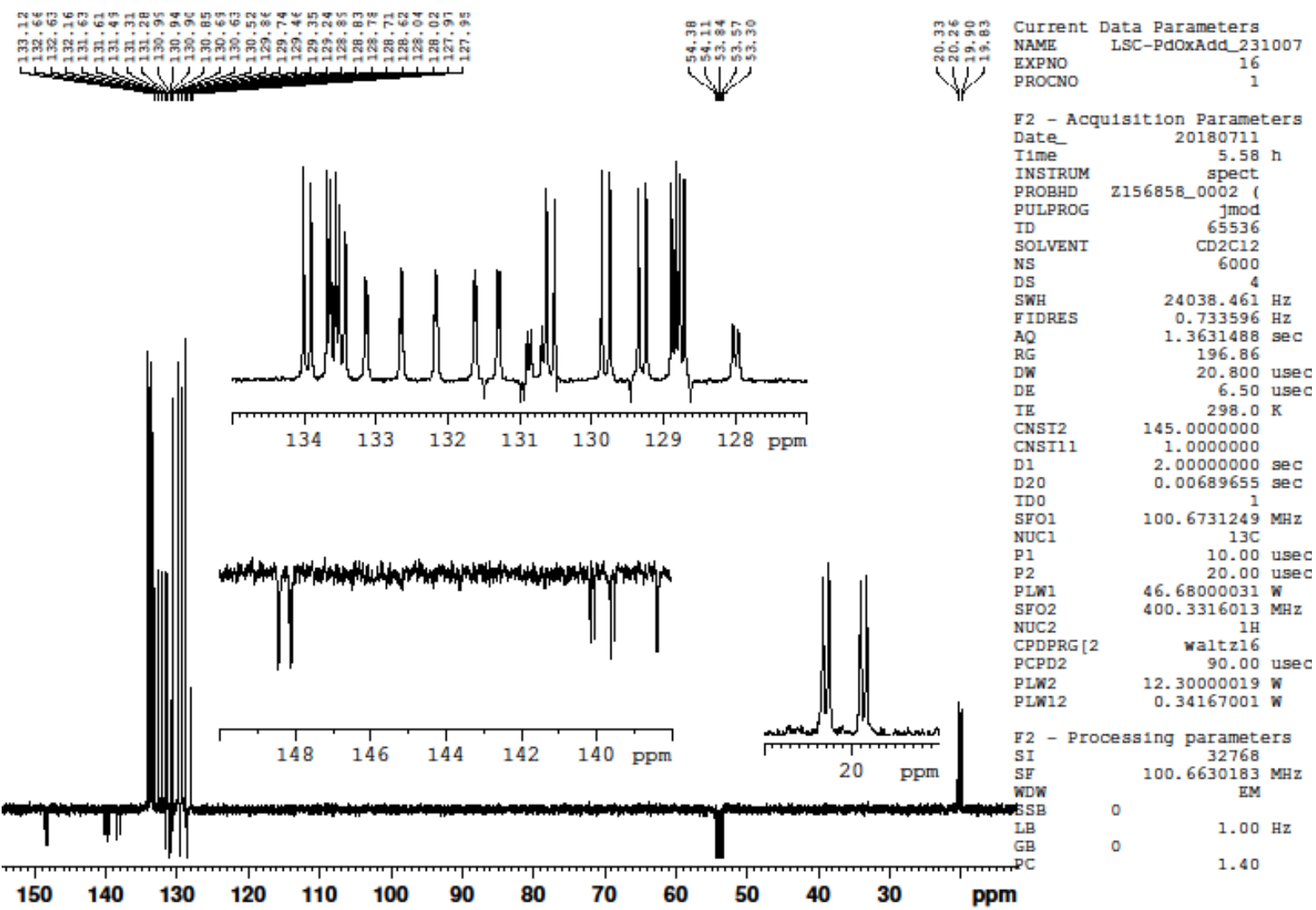

Figure S15. $\left\{{ }^{1} \mathrm{H}\right\}{ }^{13} \mathrm{C}$ APT NMR spectrum of 1-PdCl.

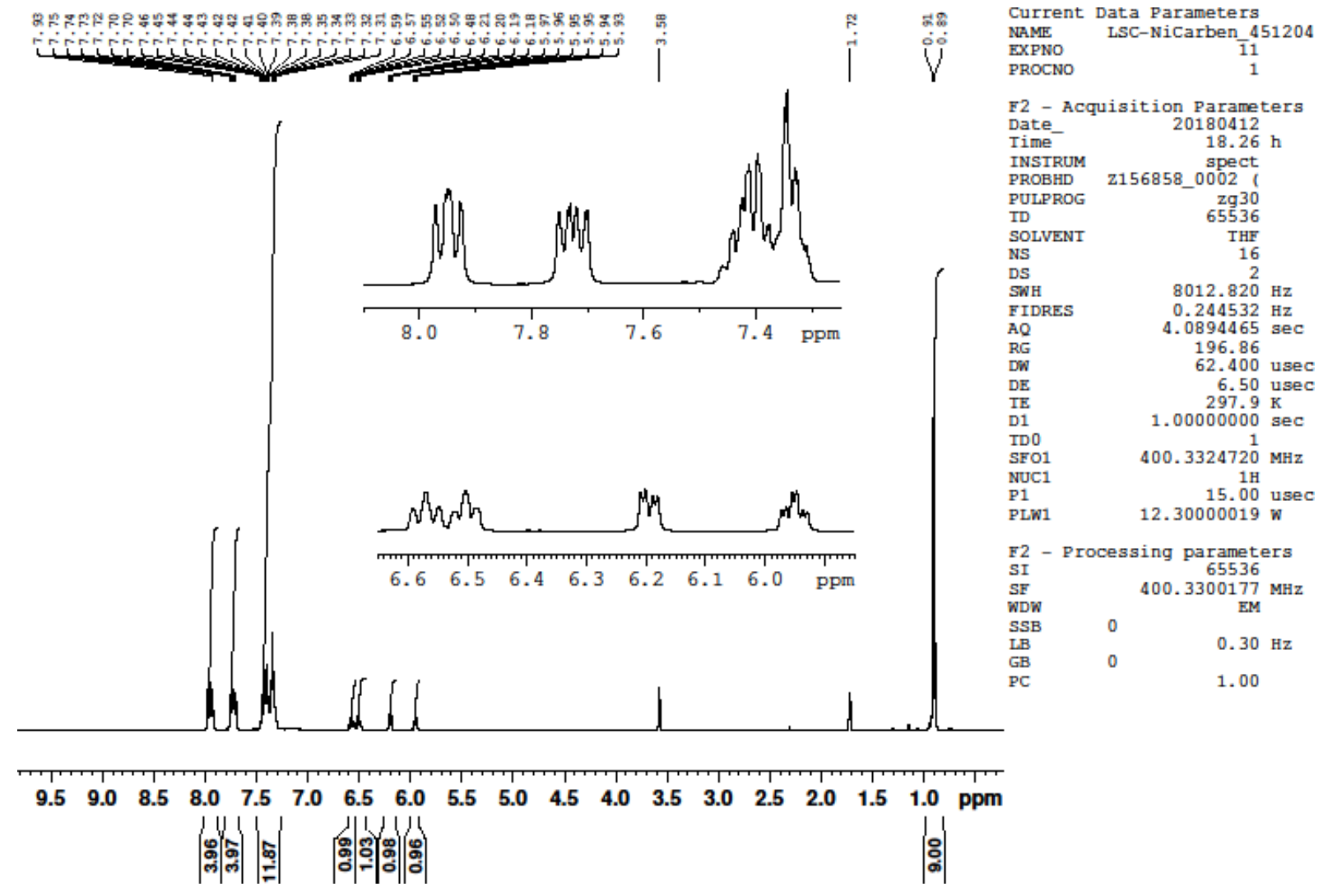

Figure S16. ${ }^{1} \mathrm{H}$ NMR spectrum of 1-Ni. 

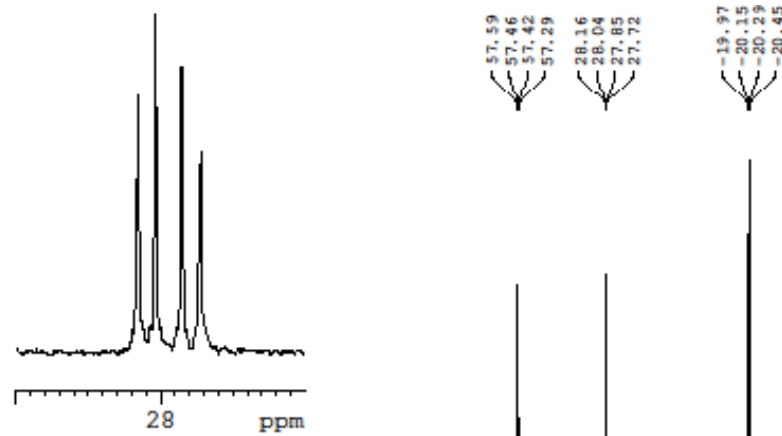

Current Data Parameters
NAME
LXSC-NiCarben_451204
EXPNO

PROCNO

F2 - Acquisition Parameters

Date_ 20180412

IIme 18.23

INSTRUM
PROBHD

PULPROG 2156858_o zgpg30

ID 65536

SOLNENT

NS

$\begin{array}{lr}\text { DS } & 4 \\ \text { SWH } & 64102.562 \mathrm{~Hz}\end{array}$

0.5111808 HZ

$\begin{array}{lr}\text { AQ } & 0.5111808 \\ \text { RG } & 196.86\end{array}$

$\begin{array}{lr}\mathrm{DW} & 7.800 \text { usec } \\ \mathrm{DE} & 6.50 \text { usec }\end{array}$

IE

D1 $2.00000000 \mathrm{sec}$

D11 $0.03000000 \mathrm{sec}$

SFO1 $\quad 162.0646573 \mathrm{MHz}$

NUC

$\mathrm{P} 1$

$\begin{array}{lr}\text { P1 } & 15.00 \text { usec } \\ \text { PLw1 } & 10.93400002 \mathrm{w}\end{array}$

$400.3316013 \mathrm{MH}$

NUC2 $1 \mathrm{H}$

CPDPRG [ 2 waltz16

PCPD2 90.00 usec

PLW12 $\quad 0.34167001 \mathrm{~W}$

DL13 0.17185999 \%

F2 - Processing parameters

$\begin{array}{lr}\text { SI } & 32768 \\ \text { SF } & 162.0565544 \\ \text { MHz }\end{array}$ $\begin{array}{lr}\text { SF } & 162.0565544 \\ \text { WDW } & \text { EM M }\end{array}$

SSB $\quad 0$

$\begin{array}{lll}\mathrm{SSB} & 0 & 1.00 \mathrm{~Hz}\end{array}$

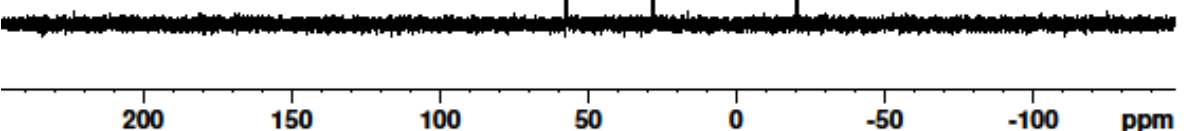

1.40

Figure S17. ${ }^{31} \mathrm{P}$ NMR spectrum of $\mathbf{1}-\mathbf{N i}$.

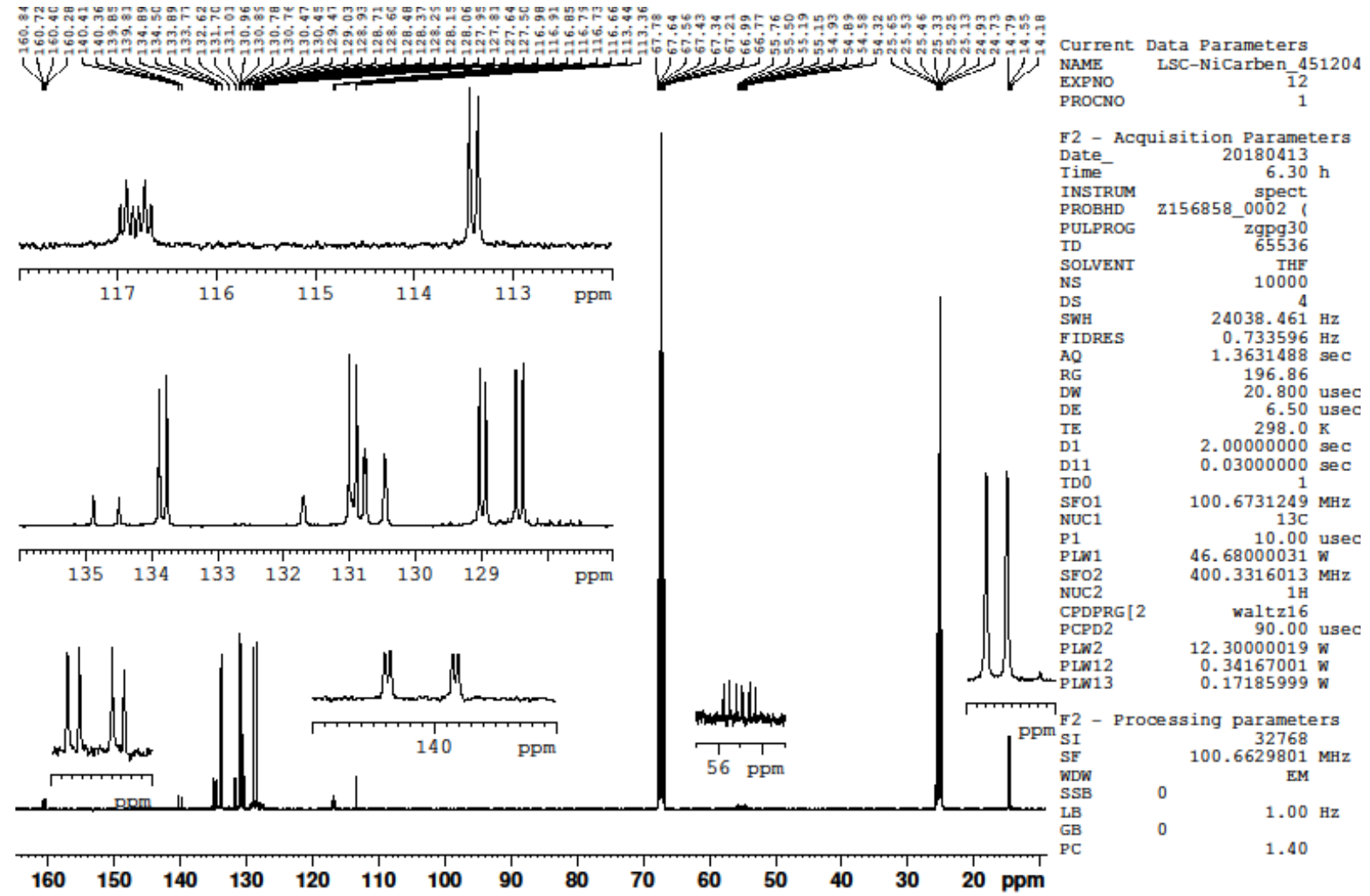

Figure S18. $\left\{{ }^{1} \mathrm{H}\right\}{ }^{13} \mathrm{C}$ NMR spectrum of 1-Ni. 


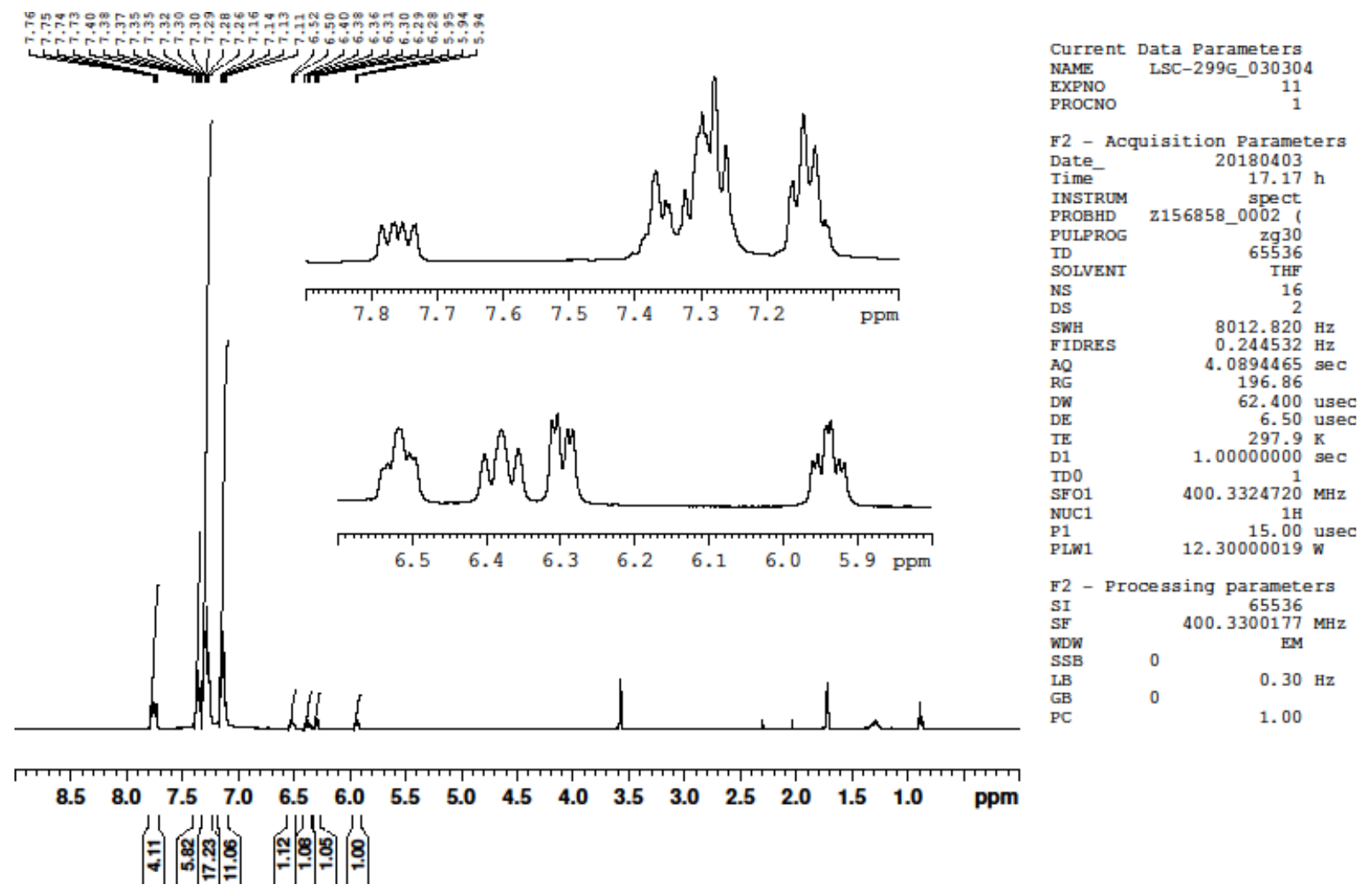

Figure S19. ${ }^{1} \mathrm{H}$ NMR spectrum of 1-Pd (unlabelled peaks between 0 and 2 ppm belong to residual pentane).

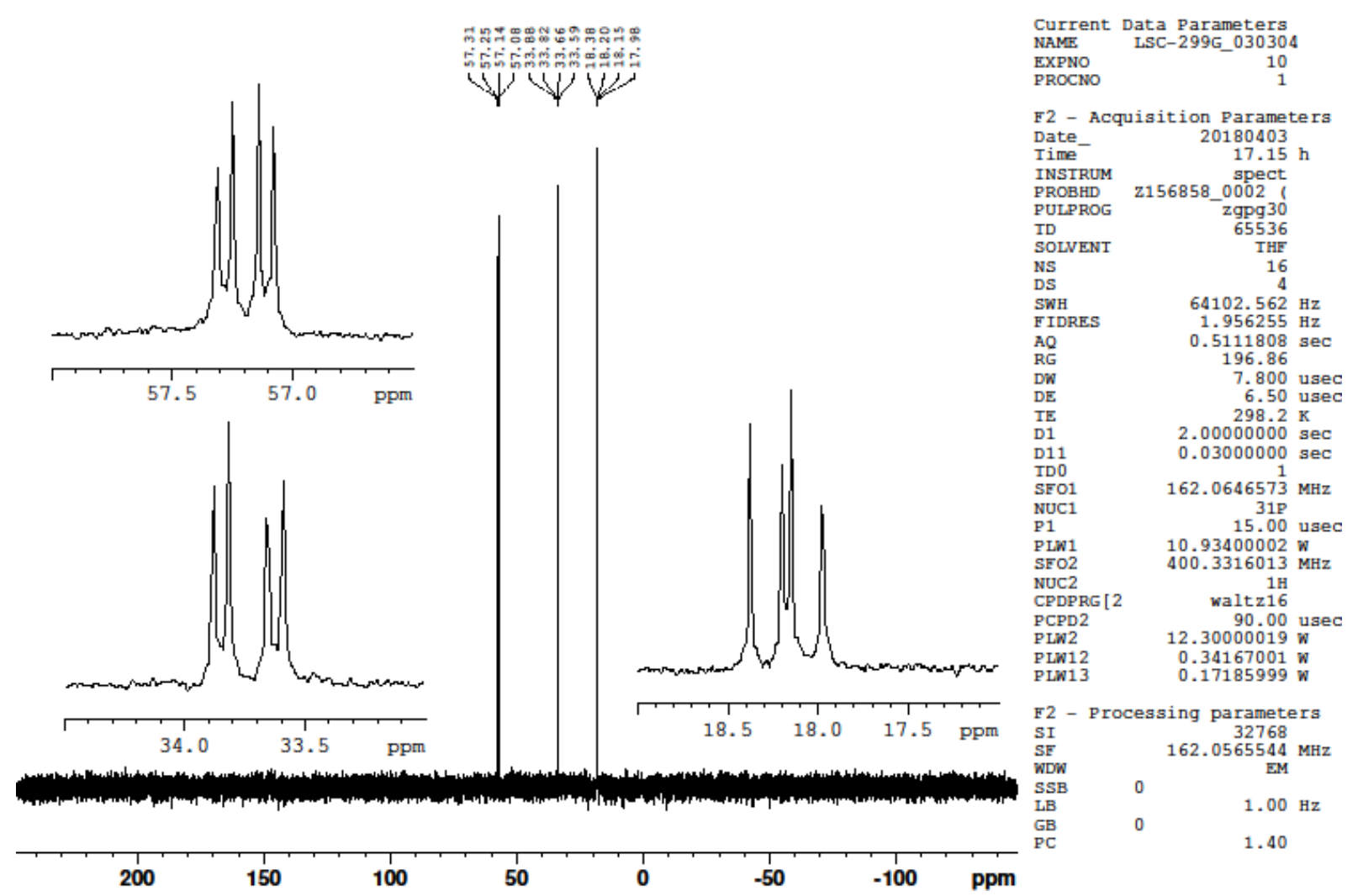

Figure S20. ${ }^{31} \mathrm{P}$ NMR spectrum of 1-Pd. 


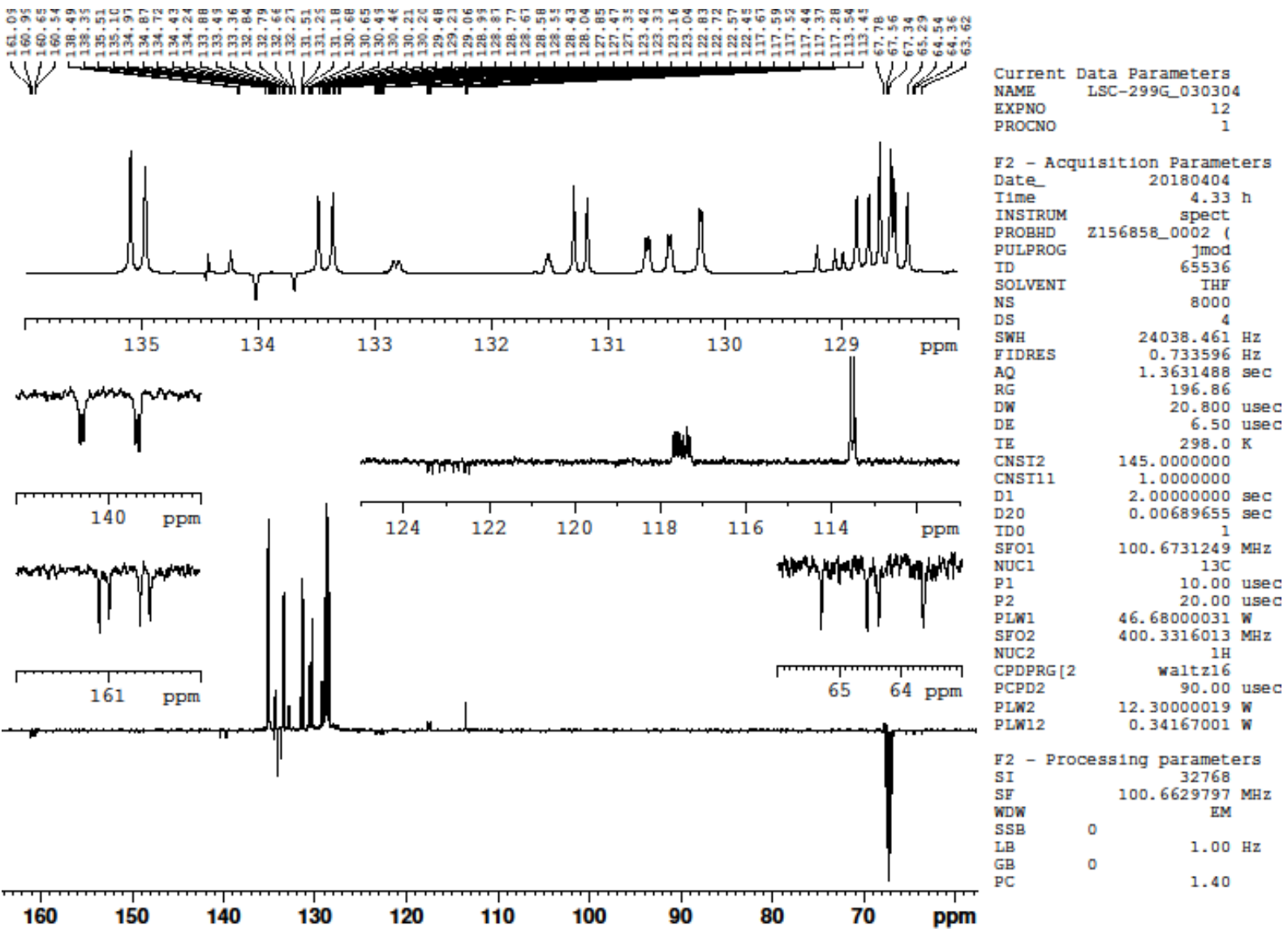

Figure S21. $\left\{{ }^{1} \mathrm{H}\right\}{ }^{13} \mathrm{C}$ APT NMR spectrum of 1-Pd.
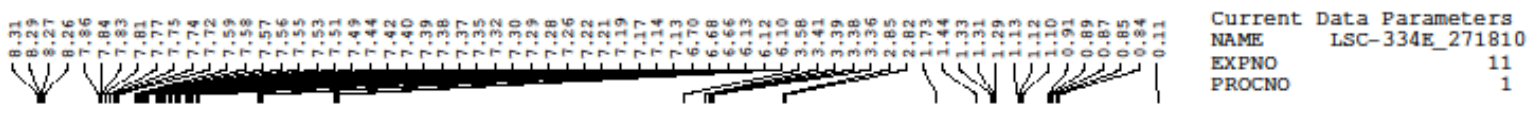

EXPNO

F2 - Acquisition Parameters

Date_ 20181018

Time 18.00

INSTRUM spect

Z156858_0002

TD $\quad 65536$

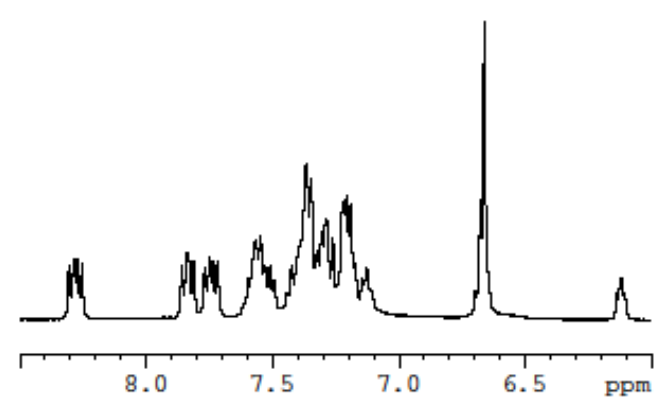

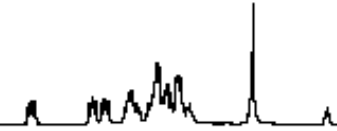

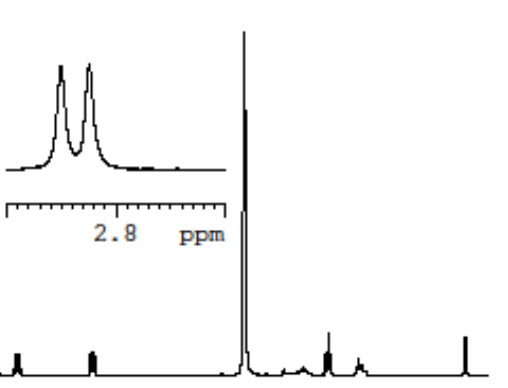

TD

NS

SWH

$8012.820 \mathrm{~Hz}$

$4.0894465 \mathrm{sec}$

RG

62.400 usec

6.50 usec

$00000000 \mathrm{~K}$

400. $3324720 \mathrm{MHz}$

$1 \mathrm{H}$

15.00 usec
$12.30000019 \mathrm{w}$

LW1

F2 - Processing parameters

SI 65536

$\begin{array}{ll}\text { SF } & 400.3300147 \text { MHz }\end{array}$

SSB $\quad 0$

$\begin{array}{lll}\mathrm{LB} & 0.30 \mathrm{~Hz}\end{array}$

GB

1.00

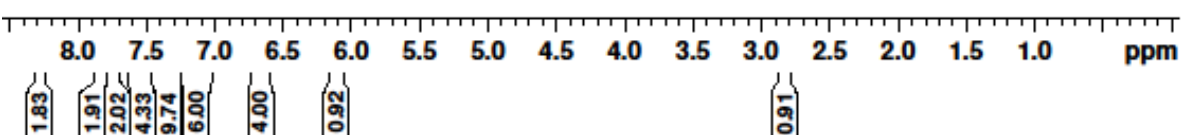

Figure S22. ${ }^{1} \mathrm{H}$ NMR spectrum of 4 (unlabelled peaks between 0 and $3.5 \mathrm{ppm}$ belong to residual pentane, diethylether and grease). 


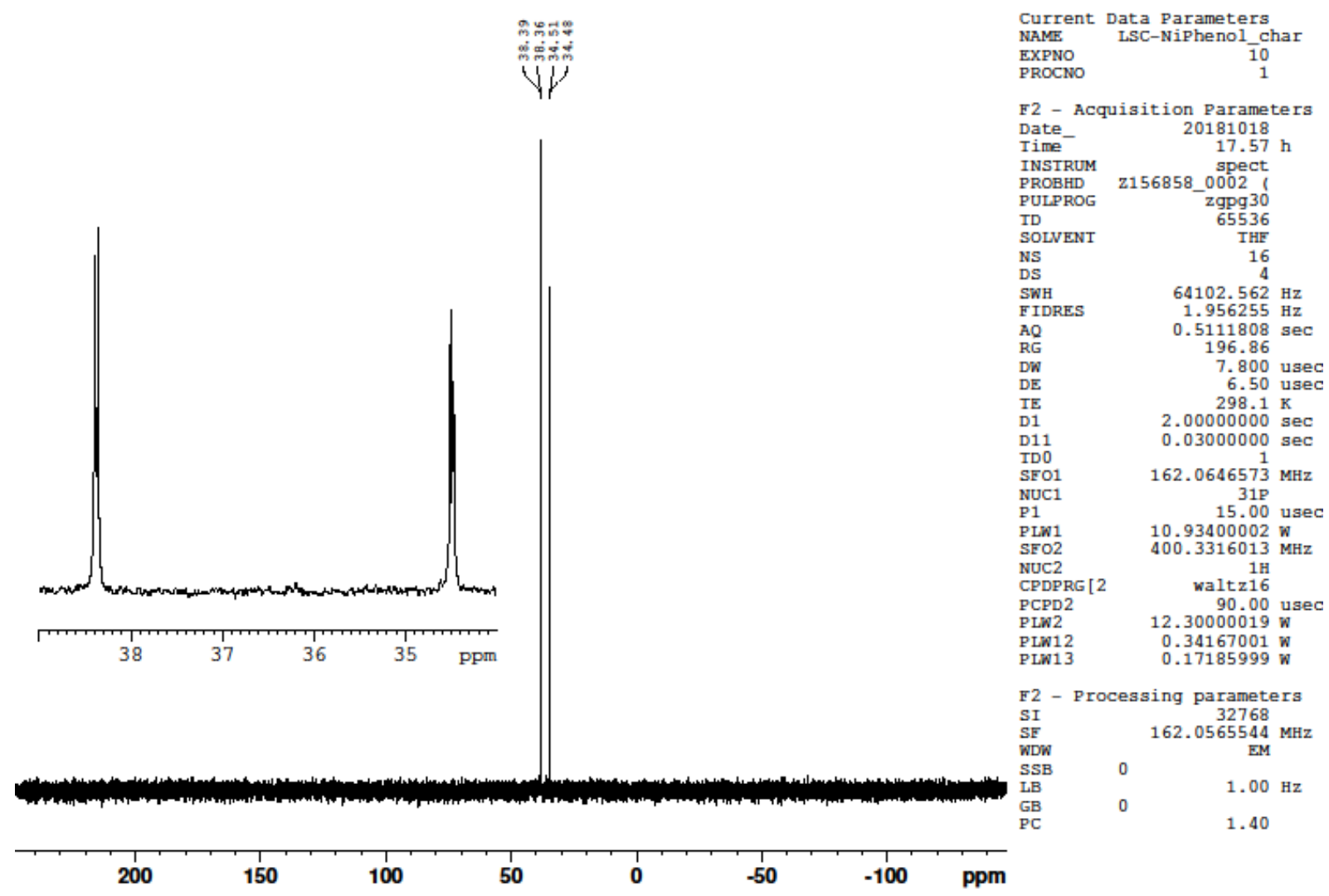

Figure S23. ${ }^{31} \mathrm{P}$ NMR spectrum of 4.
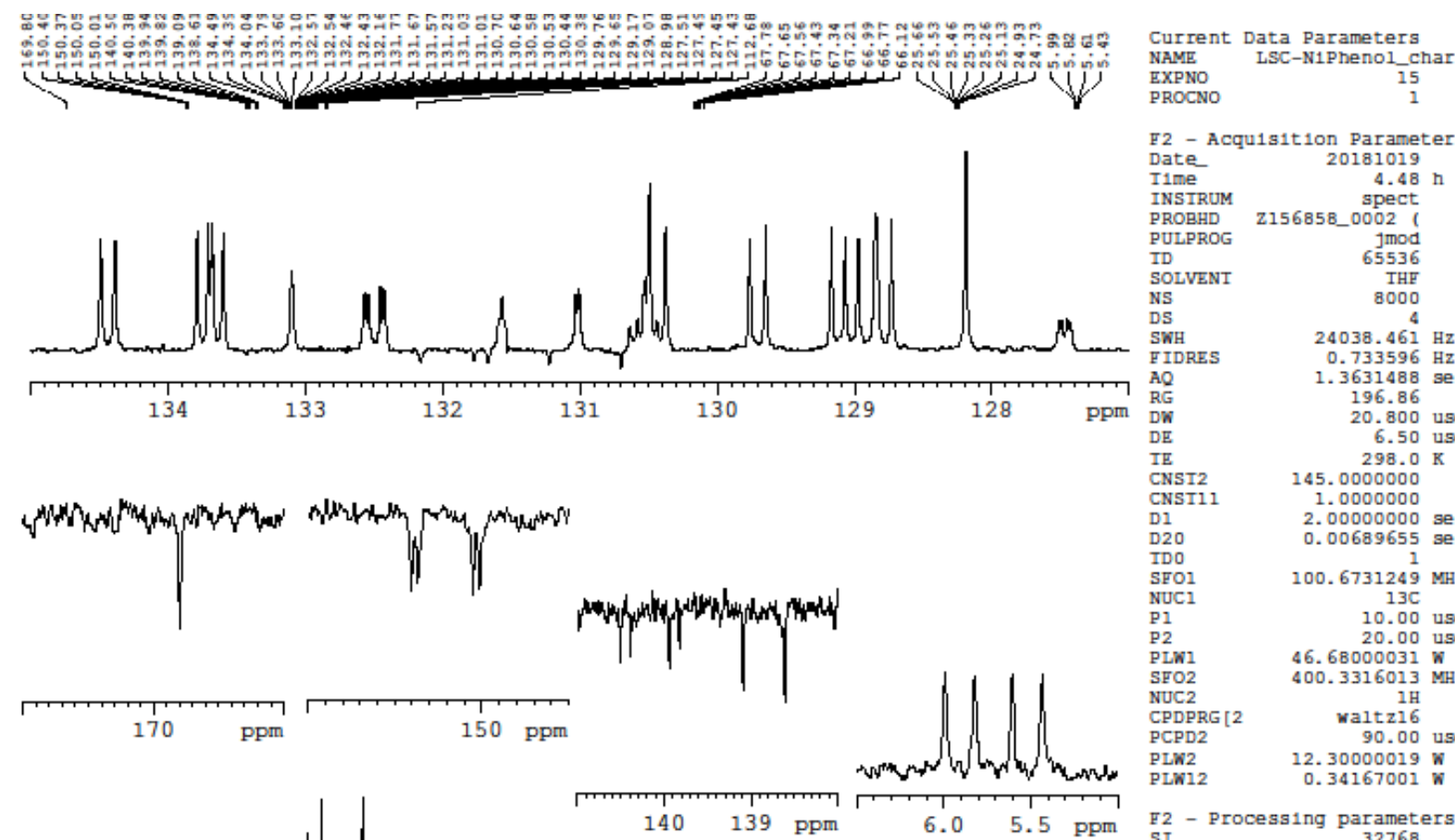

F2 - Acqu1s1tion Parameters Date- 20181019 I1me 4.48 INSIRUM spect PROBHD Z156858_0002 PULPROG Jmod SOLVENT NS
DS
SWH
FIDR TIDRES $24038.461 \mathrm{~Hz}$ AQ $\quad 1.3631488 \mathrm{sec}$ RG
$D W$
$D E$
IE 1.36 .86
196.800 20.800 usec
6.50 usec $298.0 \mathrm{~K}$ CNST2 $\quad 145.0000000$ CNST11 $\quad 1.0000000$ D1 2.00000000 sec D20 $0.00689655 \mathrm{sec}$ $\begin{array}{ll}\text { IDO } & 1 \\ \text { SFO1 } & 100.6731249 \mathrm{MHz}\end{array}$ $\begin{array}{lr}\text { SFO1 } & 100.6731249 \mathrm{MHz} \\ \text { NUC1 } & 13 \mathrm{C}\end{array}$ $\begin{array}{ll}\text { NUC1 } & 13 \mathrm{C} \\ \mathrm{P} 1 & 10.00 \text { usec }\end{array}$ PLW1 46.68000031 $\mathrm{w}$ $\mathrm{STO} 2$
$\mathrm{NUC2}$ CPDPRG [2 waltzit PCPD2 $\quad 90.00$ usec PLW2 12.30000019 $\mathrm{w}$

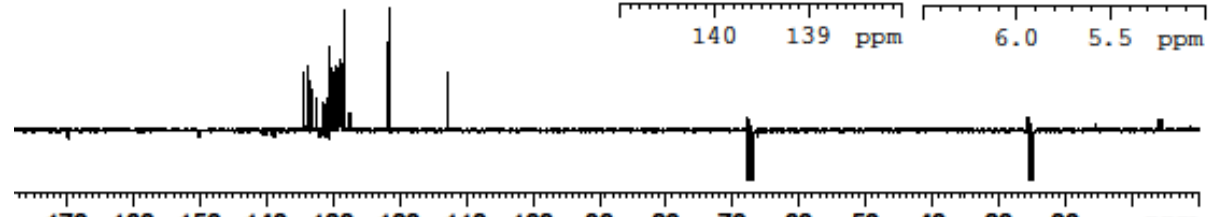
$\begin{array}{lcc}\text { F2 } & \text { Processing parameters } \\ \text { SI } & 32768 \\ \text { SF } & & 100.6629794 \mathrm{MHz} \\ \text { WDW } & 0 & \text { EM } \\ \text { SSB } & 0 & 1.00 \mathrm{~Hz} \\ \text { LB } & 0 & 1.40 \\ \text { GB } & 0 & \end{array}$

Figure S24. $\left\{{ }^{1} \mathrm{H}\right\}{ }^{13} \mathrm{C}$ APT NMR spectrum of 4 . 


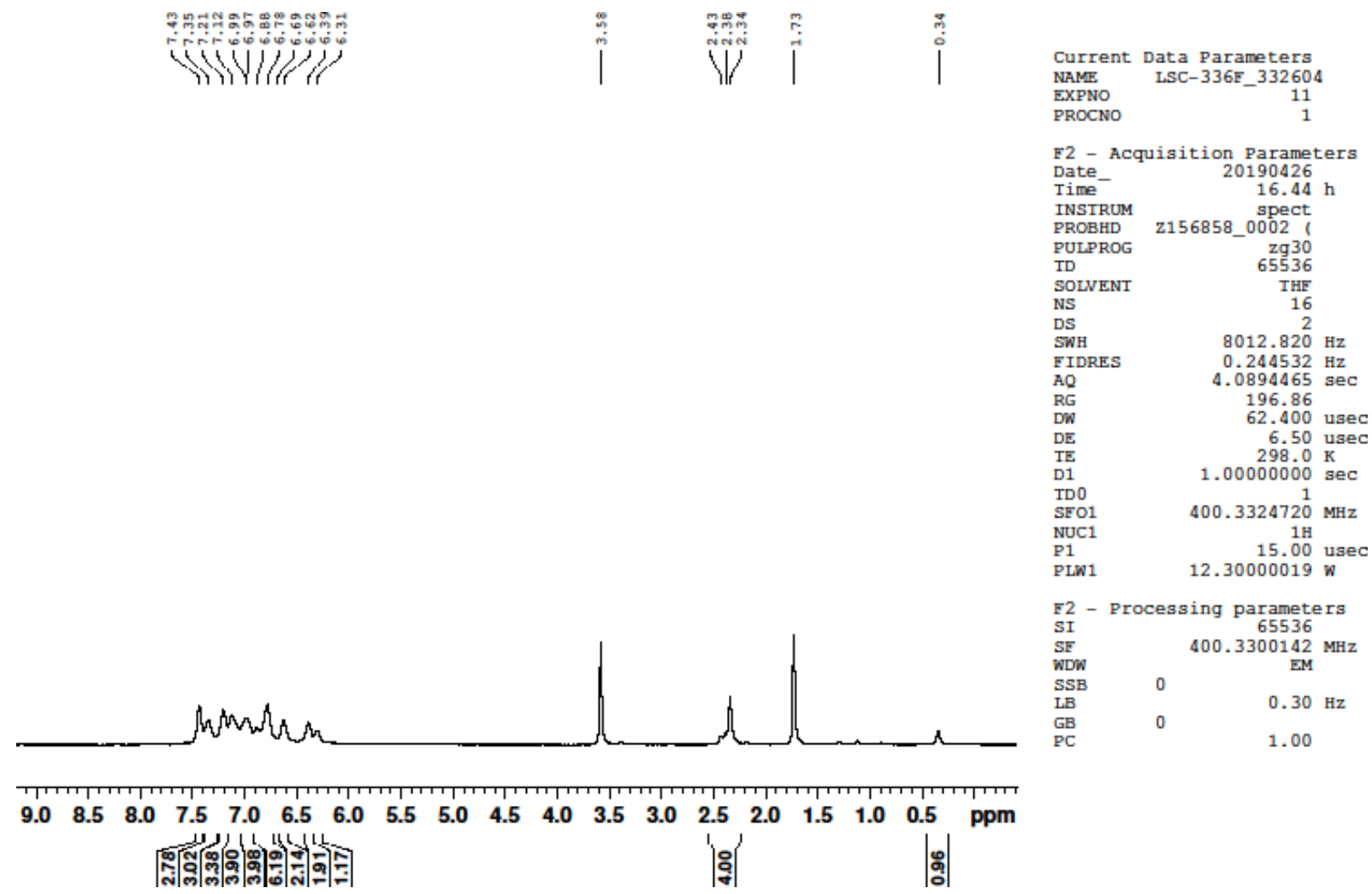

Figure S25. ${ }^{1} \mathrm{H}$ NMR spectrum of $\mathbf{5}$ (unlabelled peaks between 2.0 and $2.5 \mathrm{ppm}$ belong to residual toluene).

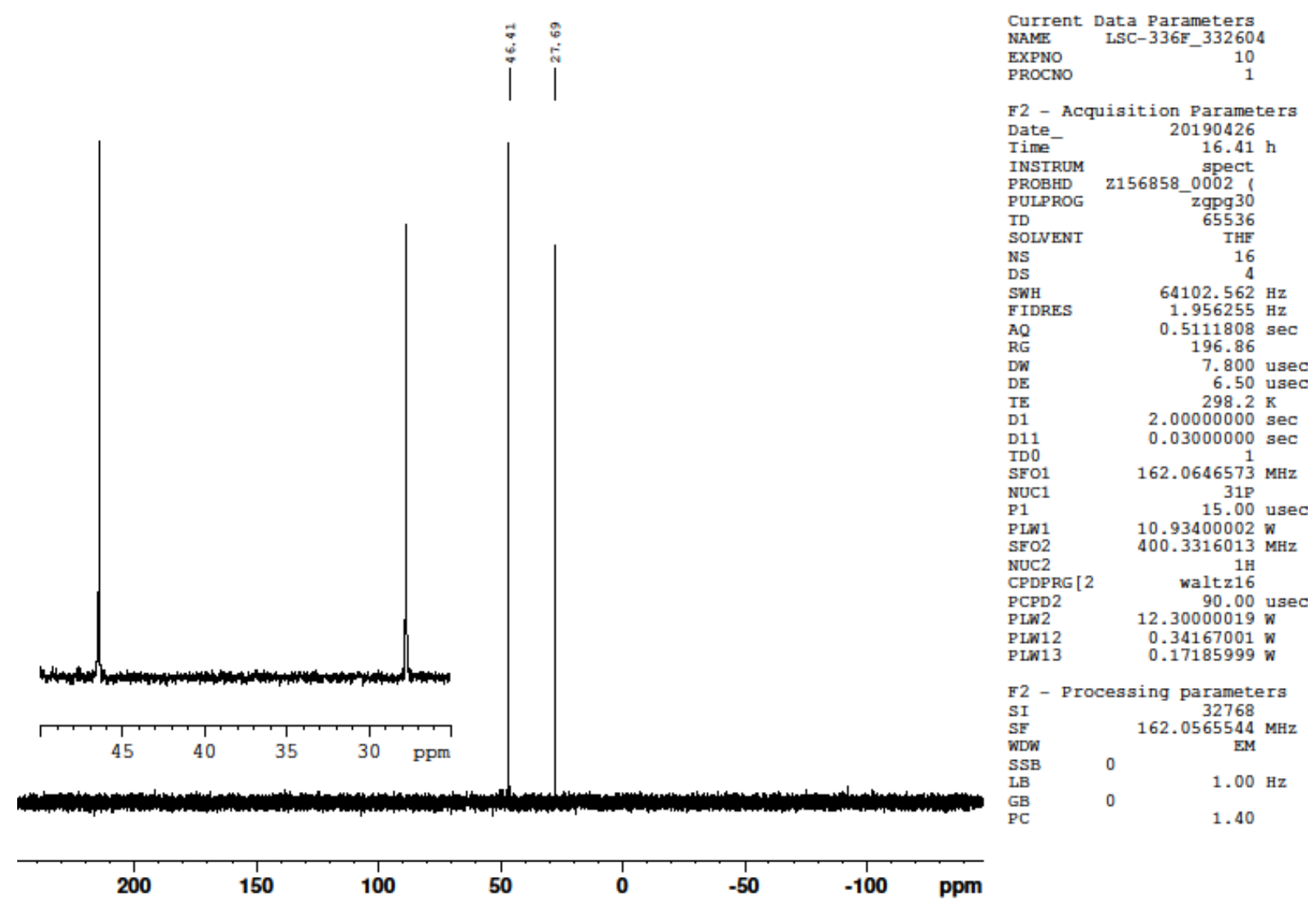

Figure S26. ${ }^{31} \mathrm{P}$ NMR spectrum of $\mathbf{5}$. 


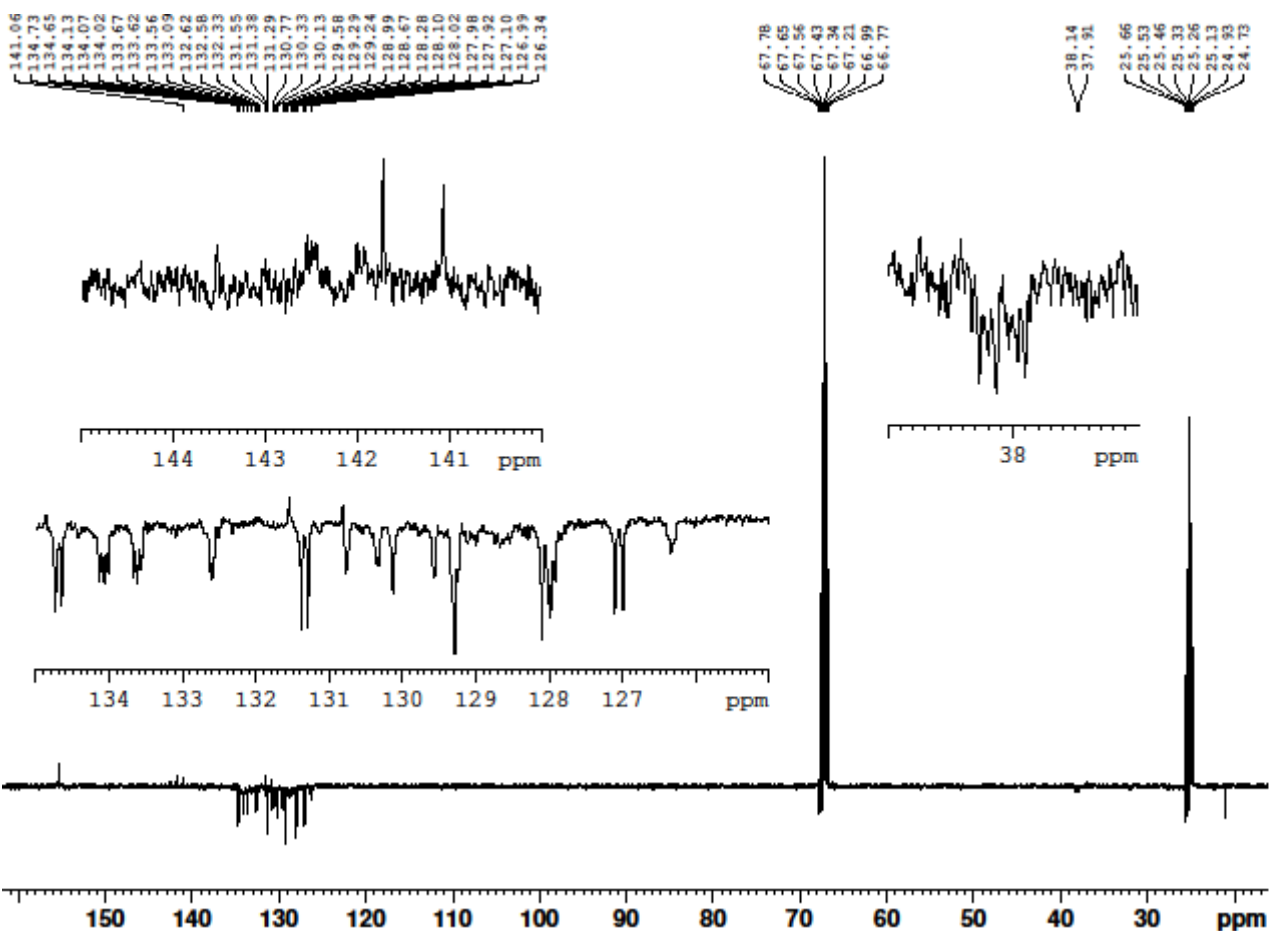

$\begin{array}{lc}\text { Current } & \text { Data Parameters } \\ \text { NaME } & \text { LSC-336F_332604 } \\ \text { EXPNO } & 13 \\ \text { PROCNO } & 1\end{array}$

72 - Acquisition Paraneter

$\begin{array}{ll}\text { Date- } & 20190427 \\ \text { Iime } & 10.17 \mathrm{~h}\end{array}$

NSTRUM spect

$\begin{array}{lr}\text { PROABD } & \text { Z156858_0002 } \\ \text { TDLPROC } & \text { jmod } \\ 65536\end{array}$

SOLVEN

DS

$\begin{array}{lr}\text { SWH } & 24038.461 \mathrm{~Hz} \\ \text { FIDRES } & 0.733596 \mathrm{~Hz}\end{array}$

$\begin{array}{ll}2 & 0.733596 \mathrm{~Hz} \\ & 1.3631488 \mathrm{ge}\end{array}$

$\begin{array}{lr}\text { RE } & 196.86 \\ \text { DW } & 20.800 \text { usec } \\ \text { DE } & 6.50 \text { ugec }\end{array}$

$\mathrm{TE} \quad 298.0 \mathrm{~K}$

CNST2 145.0000000

$\begin{array}{ll}\mathrm{D} 1 & 2.00000000 \mathrm{sec} \\ \mathrm{D} 20 & 0.00699655 \mathrm{sec}\end{array}$

TD0

SFO1 $100.6731249 \mathrm{MHZ}$

$\begin{array}{ll}\text { Nuc1 } & 13 \mathrm{C} \\ \mathrm{P} 1 & 10.00 \mathrm{usec} \\ \mathrm{P} 2 & 20.00 \mathrm{ugec}\end{array}$

$\begin{array}{lr}\text { P2 } & 20.00 \mathrm{was} \\ \text { PIW } 1 & 46.68000031 \mathrm{w}\end{array}$

NuC2 $21 \mathrm{H}$

CPDPRG [2 waltz16
90.00 ugs

$\begin{array}{lr}\text { PCPD2 } & 90.00 \text { us } \\ \text { PIW 2 } & 12.30000019 \text { w }\end{array}$

F2 - Processing parameters
SI

$\begin{array}{lr}\text { SI } & 32768 \\ \text { SI } & 100.6629781 \mathrm{MHz} \\ \text { WDW } & \text { EM }\end{array}$

Ls

Figure S27. $\left\{{ }^{1} \mathrm{H}\right\}{ }^{13} \mathrm{C}$ APT NMR spectrum of 5 . 


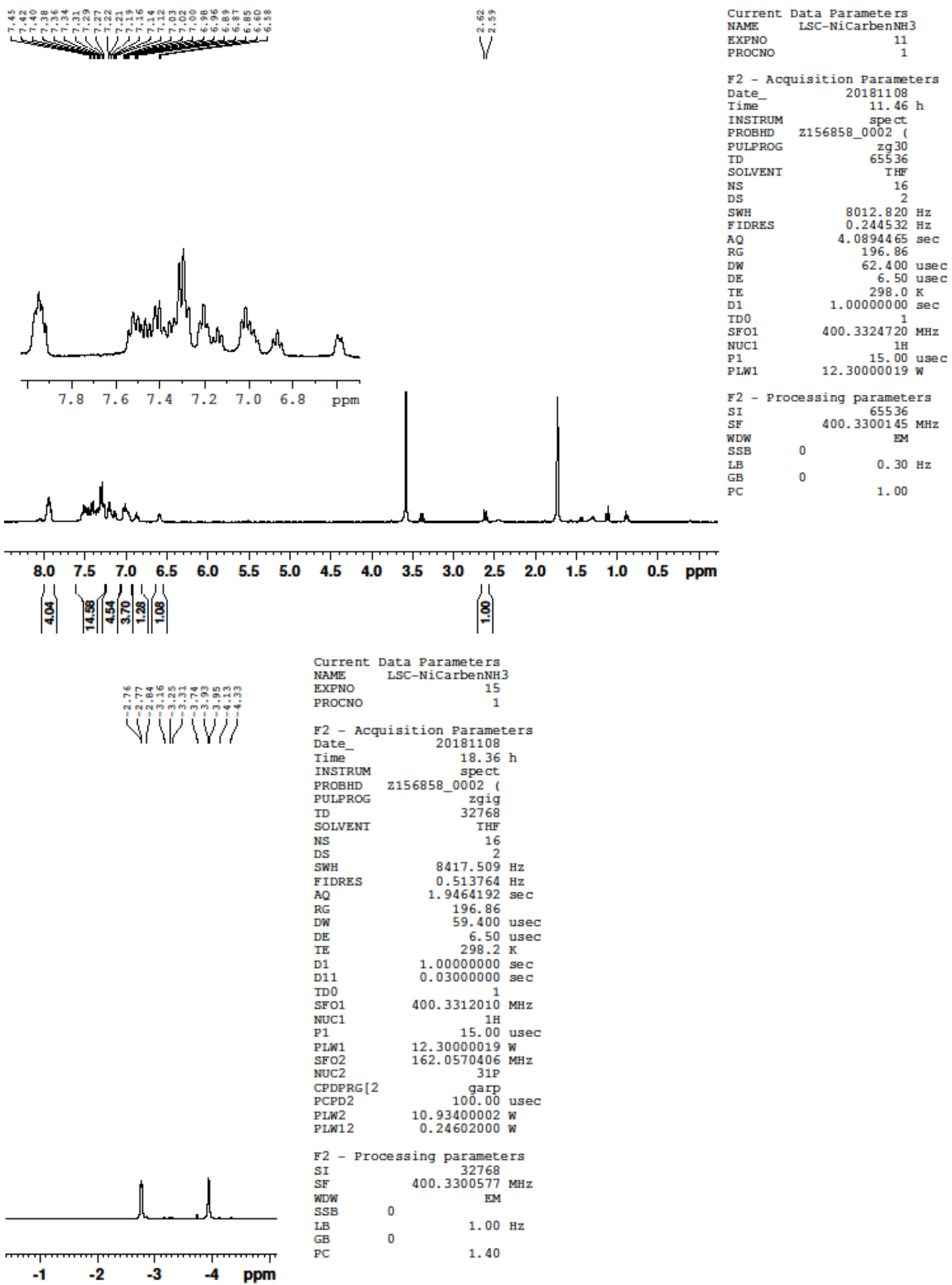

Figure S28. ${ }^{1} \mathrm{H}$ NMR spectrum of 6 (unlabelled peaks between 0.0 and $3.5 \mathrm{ppm}$ belong to residual pentane and diethylether). 


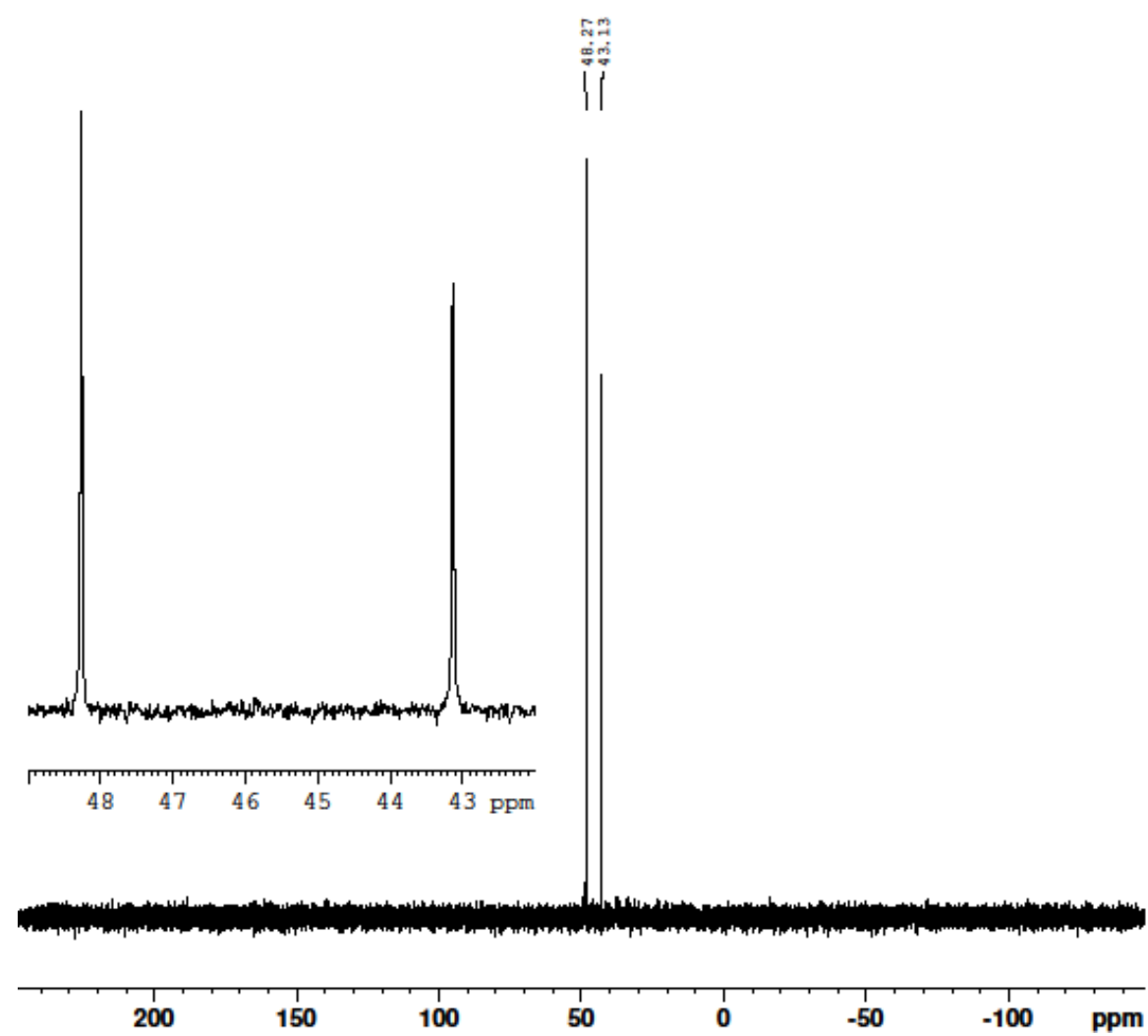

Figure S29. ${ }^{31} \mathrm{P}$ NMR spectrum of 6.

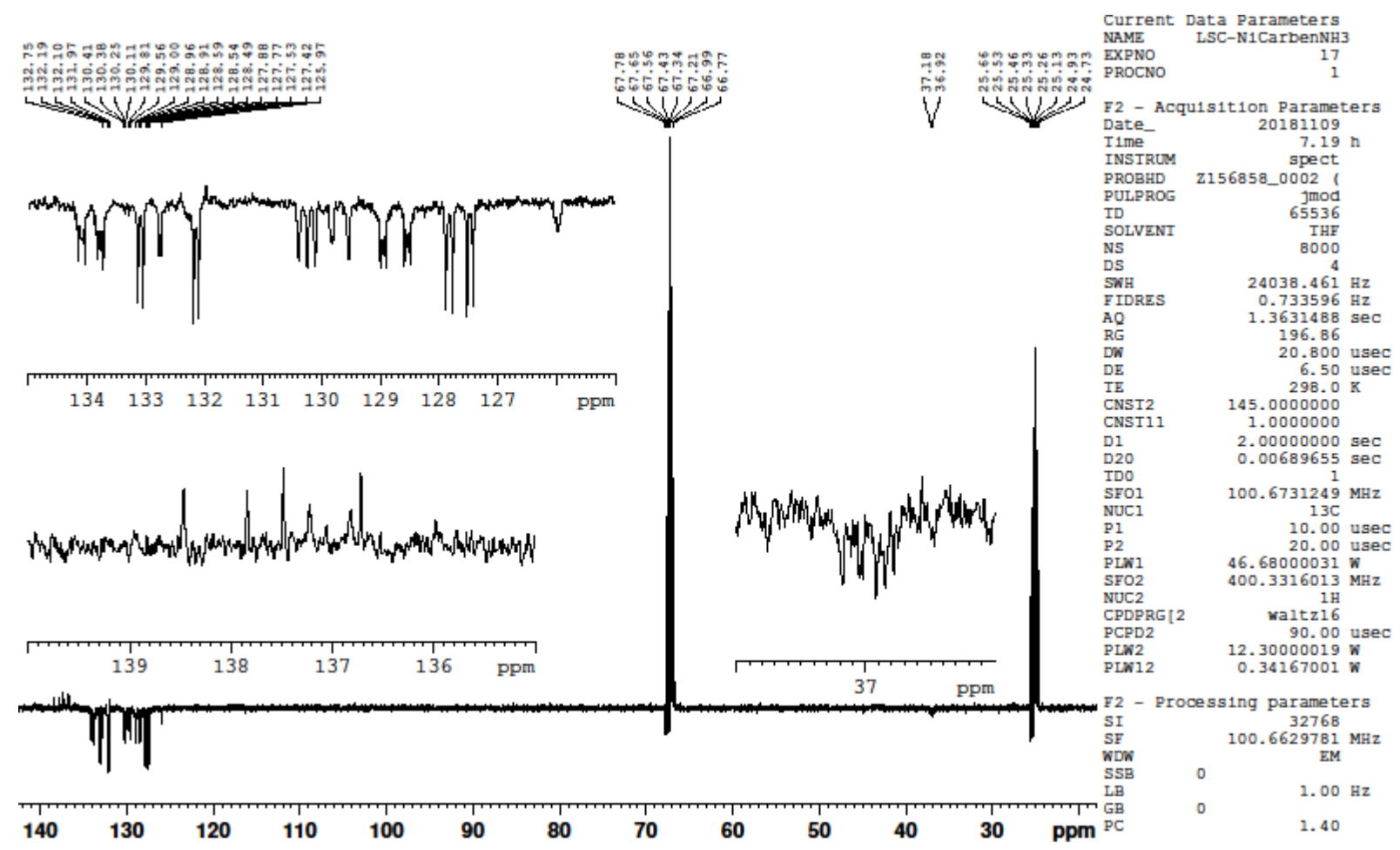

Figure S30. $\left\{{ }^{1} \mathrm{H}\right\}{ }^{13} \mathrm{C}$ APT NMR spectrum of 6.

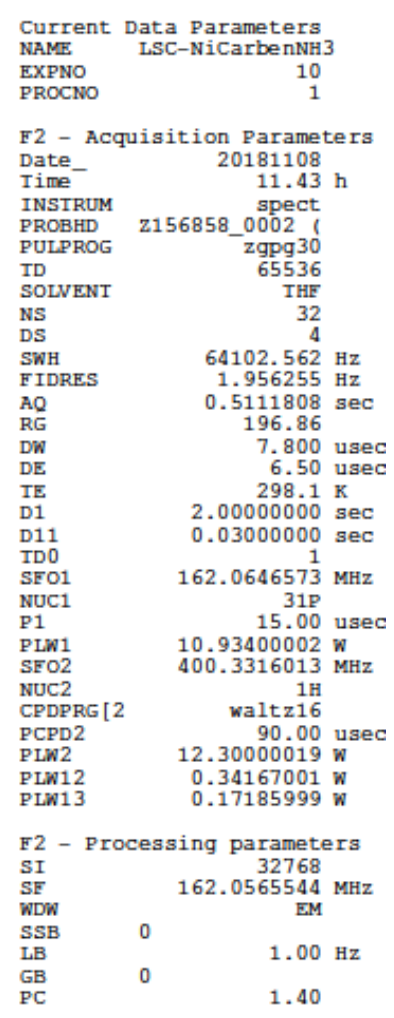




\section{Crystal structure determination}

Data collection of all compounds was conducted either with a Bruker X8-APEX II (1), Oxford Synergy $\left(4,5_{2}, \mathbf{6}_{2}\right)$ or Oxford SuperNova (1-Cl, 1-Ni, 1-NiCl, 1-Pd, 1-PdCl, 3-Ni, 3-Pd). The structures were solved using direct methods, refined with the Shelx software package ${ }^{[1]}$ and expanded using Fourier techniques. The crystals of all compounds were mounted in an inert oil (perfluoropolyalkylether). Crystal structure determinations were effected at $100 \mathrm{~K}$. Crystallographic data (including structure factors) have been deposited with the Cambridge Crystallographic Data Centre as supplementary publication no. CCDC-1921059-1921069. Copies of the data can be obtained free of charge on application to Cambridge Crystallographic Data Centre, 12 Union Road, Cambridge CB2 1EZ, UK; [fax: (+44) 1223-336-033; email: deposit@ccdc.cam.ac.uk].

Details on the structure solutions

The structure of 3-Pd contained a highly disordered solvent molecule (THF). This was modelled by using the SAME, RIGU and PART instructions. The occupancy of the two sites was 0.70:0.30.

The structure 3-Ni contained a highly disordered solvent molecule (THF). This was modelled by using the SAME and PART instructions. The occupancy of the two sites was 0.59:041.

The structure 1-PdCl contained a disordered solvent molecule (DCM) on a symmetry centre. This was modelled by using the SADI and PART instructions. Due to the special position, the occupancy was constrained to 0.5 .

The structure of $\mathbf{6}_{\mathbf{2}}$ contained a highly disordered solvent molecule (THF). This was modelled by using the SAME, RIGU and PART instructions. The occupancy of the two sites was 0.63:0.37. Additionally, a disordered phenyl moiety was present. This was modelled by using the SAME, RIGU and PART instructions as well as one EADP instruction. The occupancy of the two sites was 0.70:0.30.

\subsection{Crystal Tables}

Table S1. Data collection and structure refinement details for compounds 1, 1-Cl and 1-NiCl.

\begin{tabular}{cccc}
\hline & $\mathbf{1}$ & $\mathbf{1 - C l}$ & $\mathbf{1 - N i C l}$ \\
\hline CCDC No. & 1921059 & 1921060 & 1921062 \\
\hline Empirical formula & $\mathrm{C}_{31} \mathrm{H}_{26} \mathrm{P}_{2} \mathrm{~S}$ & $\mathrm{C}_{31} \mathrm{H}_{25} \mathrm{ClP}_{2} \mathrm{~S}$ & $\mathrm{C}_{31} \mathrm{H}_{25} \mathrm{ClNiP}_{2} \mathrm{~S}$ \\
\hline Molecular weight $\left[\mathrm{g} \cdot \mathrm{mol}^{-1}\right]$ & 492.52 & 526.96 & 585.67 \\
\hline Temperature $[\mathrm{K}]$ & $100(2)$ & $100(2)$ & $100.05(10)$ \\
\hline Wavelength $[\AA]$ & 0.71073 & 1.54184 & 1.54184 \\
\hline Crystal system & Monoclinic & Orthorhombic & Monoclinic \\
\hline Space group & $P 2{ }_{1} / \mathrm{c}$ & $P$ na2 & $P 21 / \mathrm{c}$ \\
$\mathrm{a}[\AA]$ & $22.1636(14)$ & $8.82363(9)$ & $9.6112(3)$ \\
$\mathrm{b}[\AA]$ & $9.9017(6)$ & $14.70752(16)$ & $12.4518(4)$ \\
$\mathrm{c}[\AA]$ & $11.2272(7)$ & $20.7947(2)$ & $22.4474(8)$ \\
$\alpha\left[^{\circ}\right]$ & 90 & 90 & 90 \\
$\beta\left[{ }^{\circ}\right]$ & $93.012(2)$ & 90 & $98.622(3)$ \\
$\gamma\left[^{\circ}\right]$ & 90 & 90 & 90 \\
$\mathrm{Volume}\left[\AA^{3}\right]$ & $2460.5(3)$ & $2698.61(5)$ & $2656.06(15)$
\end{tabular}




\begin{tabular}{|c|c|c|c|}
\hline Z & 4 & 4 & 4 \\
\hline Density (calculated) $\left[\mathrm{Mg} \cdot \mathrm{m}^{3}\right]$ & 1.330 & 1.297 & 1.465 \\
\hline Absorption coefficient $\left[\mathrm{mm}^{-1}\right]$ & 0.280 & 3.227 & 3.996 \\
\hline $\mathrm{F}(000)$ & 1032 & 1096 & 1208 \\
\hline Crystal size $[\mathrm{mm}]$ & $0.180 \times 0.130 \times 0.110$ & $0.253 \times 0.198 \times 0.055$ & $0.153 \times 0.119 \times 0.077$ \\
\hline$\theta$ range for data collection $\left[{ }^{\circ}\right]$ & 1.840 to 24.997 & 3.681 to 76.233 & 3.984 to 74.507 \\
\hline Index ranges & $\begin{aligned}-26 & \leq \mathrm{h} \leq 26 \\
-11 & \leq \mathrm{k} \leq 11 \\
-13 & \leq 1 \leq 13\end{aligned}$ & $\begin{array}{c}-11 \leq \mathrm{h} \leq 9 \\
-18 \leq \mathrm{k} \leq 18 \\
-25 \leq 1 \leq 25\end{array}$ & $\begin{aligned}-11 & \leq \mathrm{h} \\
-15 & \leq \mathrm{k} \leq 12 \\
-27 & \leq 1 \leq 28\end{aligned}$ \\
\hline Reflections collected & 35859 & 25217 & 27133 \\
\hline Independent reflections & $4335[\mathrm{R}(\mathrm{int})=0.0361]$ & $5428[\mathrm{R}(\mathrm{int})=0.0302]$ & $5429[\mathrm{R}(\mathrm{int})=0.0236]$ \\
\hline Data / restraints / parameters & $4335 / 0 / 315$ & $5428 / 1 / 320$ & $5429 / 0 / 400$ \\
\hline Goodness-of-fit on $\mathrm{F}^{2}$ & 1.040 & 1.036 & 1.023 \\
\hline Final $R$ indices $[\mathrm{I}>2 \sigma(\mathrm{I})]$ & $\begin{array}{c}\mathrm{R} 1=0.0291 \\
\mathrm{wR} 2=0.0689\end{array}$ & $\begin{array}{c}\mathrm{R} 1=0.0255 \\
\mathrm{wR} 2=0.0667\end{array}$ & $\begin{array}{c}\mathrm{R} 1=0.0241 \\
\mathrm{wR} 2=0.0609\end{array}$ \\
\hline $\mathrm{R}$ indices (all data) & $\begin{array}{c}\mathrm{R} 1=0.0364 \\
\mathrm{wR} 2=0.0732\end{array}$ & $\begin{array}{c}\mathrm{R} 1=0.0259 \\
\mathrm{wR} 2=0.0670\end{array}$ & $\begin{array}{c}\mathrm{R} 1=0.0257 \\
\mathrm{wR} 2=0.0622\end{array}$ \\
\hline Largest diff. peak and hole $\left[\mathrm{e} \cdot \AA^{-3}\right]$ & 0.329 and -0.299 & 0.268 and -0.221 & 0.325 and -0.285 \\
\hline
\end{tabular}


Table S2. Data collection and structure refinement details for compounds 1-PdCl, 1-Ni, 1-Pd

\begin{tabular}{|c|c|c|c|}
\hline & 1-PdCl & $1-\mathrm{Ni}$ & 1-Pd \\
\hline CCDC No. & 1921064 & 1921061 & 1921063 \\
\hline Empirical formula & $\mathrm{C}_{31.50} \mathrm{H}_{26} \mathrm{Cl}_{2} \mathrm{P}_{2} \mathrm{PdS}$ & $\mathrm{C}_{34} \mathrm{H}_{33} \mathrm{NiP}_{3} \mathrm{~S}$ & $\mathrm{C}_{49} \mathrm{H}_{39} \mathrm{P}_{3} \mathrm{PdS}$ \\
\hline Molecular weight $\left[\mathrm{g} \cdot \mathrm{mol}^{-1}\right]$ & 675.82 & 625.28 & 859.17 \\
\hline Temperature $[\mathrm{K}]$ & $100(2)$ & $100(2)$ & $99.8(2)$ \\
\hline Wavelength $[\AA]$ & 1.54184 & 1.54184 & 1.54184 \\
\hline Crystal system & Triclinic & Monoclinic & Triclinic \\
\hline Space group & $P-1$ & $P 2_{1} / \mathrm{c}$ & $P-1$ \\
\hline $\mathrm{a}[\AA]$ & $10.0360(5)$ & $11.4768(2)$ & $16.0568(7)$ \\
\hline $\mathrm{b}[\AA]$ & $12.0549(6)$ & $18.8153(3)$ & $16.2709(9)$ \\
\hline $\mathrm{c}[\AA]$ & $12.8197(6)$ & $14.5406(3)$ & $16.9390(7)$ \\
\hline$\alpha\left[^{\circ}\right]$ & $68.714(5)$ & 90 & $72.694(4)$ \\
\hline$\beta\left[^{\circ}\right]$ & $78.739(4)$ & $108.381(2)$ & $82.871(3)$ \\
\hline$\gamma\left[^{\circ}\right]$ & $88.835(4)$ & 90 & $68.608(4)$ \\
\hline Volume $\left[\AA^{3}\right]$ & $1415.17(13)$ & 2979.71(10) & $3933.4(3)$ \\
\hline Z & 2 & 4 & 4 \\
\hline Density (calculated) $\left[\mathrm{Mg} \cdot \mathrm{m}^{3}\right]$ & 1.586 & 1.394 & 1.451 \\
\hline Absorption coefficient $\left[\mathrm{mm}^{-1}\right]$ & 8.940 & 3.282 & 5.719 \\
\hline $\mathrm{F}(000)$ & 682 & 1304 & 1760 \\
\hline Crystal size $[\mathrm{mm}]$ & $0.136 \times 0.076 \times 0.067$ & $0.101 \times 0.096 \times 0.042$ & $0.125 \times 0.084 \times 0.012$ \\
\hline$\theta$ range for data collection $\left[{ }^{\circ}\right]$ & 3.778 to 67.068 & 3.973 to 74.374 & 3.425 to 67.078 \\
\hline \multirow{3}{*}{ Index ranges } & $-11 \leq \mathrm{h} \leq 11$ & $-14 \leq \mathrm{h} \leq 13$ & $-19 \leq \mathrm{h} \leq 18$ \\
\hline & $-14 \leq \mathrm{k} \leq 13$ & $-23 \leq \mathrm{k} \leq 19$ & $-19 \leq \mathrm{k} \leq 16$ \\
\hline & $-15 \leq 1 \leq 11$ & $-17 \leq 1 \leq 18$ & $-20 \leq 1 \leq 20$ \\
\hline Reflections collected & 9160 & 21139 & 27360 \\
\hline Independent reflections & $5031[\mathrm{R}(\mathrm{int})=0.0228]$ & $5981[\mathrm{R}($ int $)=0.0348]$ & $14035[\mathrm{R}(\mathrm{int})=0.0407]$ \\
\hline Data / restraints / parameters & $5031 / 1 / 356$ & $5981 / 0 / 355$ & $14035 / 0 / 973$ \\
\hline Goodness-of-fit on $\mathrm{F}^{2}$ & 1.034 & 1.016 & 1.012 \\
\hline Final $R$ indices $[\mathrm{I}>2 \sigma(\mathrm{I})]$ & $\begin{array}{c}\mathrm{R} 1=0.0221 \\
\mathrm{wR} 2=0.0686\end{array}$ & $\begin{array}{c}\mathrm{R} 1=0.0293 \\
\mathrm{wR} 2=0.0698\end{array}$ & $\begin{array}{c}\mathrm{R} 1=0.0372 \\
\mathrm{wR} 2=0.0866\end{array}$ \\
\hline $\mathrm{R}$ indices (all data) & $\begin{array}{c}\mathrm{R} 1=0.0232 \\
\mathrm{wR} 2=0.0594\end{array}$ & $\begin{array}{c}\mathrm{R} 1=0.0380 \\
\mathrm{wR} 2=0.0741\end{array}$ & $\begin{array}{c}\mathrm{R} 1=0.0523 \\
\mathrm{wR} 2=0.0954\end{array}$ \\
\hline Largest diff. peak and hole $\left[\mathrm{e} \cdot \AA^{-3}\right]$ & 0.709 and -0.660 & 0.443 and -0.304 & 0.885 and -0.820 \\
\hline
\end{tabular}


Table S3. Data collection and structure refinement details for compounds $\mathbf{3}_{\mathrm{Ni}}, \mathbf{3}_{\mathbf{P d}}$.

\begin{tabular}{|c|c|c|}
\hline & $\mathbf{3}_{\mathrm{Ni}}$ & 3Pd \\
\hline CCDC No. & 1921065 & 1921066 \\
\hline Empirical formula & $\mathrm{C}_{70} \mathrm{H}_{64} \mathrm{Ni}_{2} \mathrm{O}_{2} \mathrm{P}_{4} \mathrm{~S}_{2}$ & $\mathrm{C}_{70} \mathrm{H}_{64} \mathrm{O}_{2} \mathrm{P}_{4} \mathrm{Pd}_{2} \mathrm{~S}_{2}$ \\
\hline Molecular weight $\left[\mathrm{g} \cdot \mathrm{mol}^{-1}\right]$ & 1242.63 & 1338.01 \\
\hline Temperature $[\mathrm{K}]$ & $100.0(4)$ & $100(2)$ \\
\hline Wavelength $[\AA]$ & 1.54184 & 1.54184 \\
\hline Crystal system & Triclinic & Triclinic \\
\hline Space group & $P-1$ & $P-1$ \\
\hline $\mathrm{a}[\AA]$ & $9.8435(4)$ & $9.8371(4)$ \\
\hline $\mathrm{b}[\AA]$ & $11.9227(3)$ & $11.9749(4)$ \\
\hline $\mathrm{c}[\AA]$ & $13.0368(3)$ & $13.2016(5)$ \\
\hline$\alpha\left[^{\circ}\right]$ & $91.486(2)$ & $93.537(3)$ \\
\hline$\beta\left[^{\circ}\right]$ & $92.009(3)$ & $91.649(3)$ \\
\hline$\gamma\left[{ }^{\circ}\right]$ & $107.014(3)$ & $106.121(4)$ \\
\hline Volume $\left[\AA^{3}\right]$ & $1461.10(8)$ & $1489.42(10)$ \\
\hline Z & 1 & 1 \\
\hline Density (calculated) $\left[\mathrm{Mg} \cdot \mathrm{m}^{3}\right]$ & 1.412 & 1.519 \\
\hline Absorption coefficient $\left[\mathrm{mm}^{-1}\right]$ & 2.869 & 6.902 \\
\hline $\mathrm{F}(000)$ & 648 & 684 \\
\hline Crystal size $[\mathrm{mm}]$ & $0.096 \times 0.044 \times 0.037$ & $0.167 \times 0.087 \times 0.062$ \\
\hline$\theta$ range for data collection $\left[{ }^{\circ}\right]$ & 3.395 to 72.246 & 3.358 to 67.063 \\
\hline \multirow{3}{*}{ Index ranges } & $-12 \leq \mathrm{h} \leq 12$ & $-11 \leq \mathrm{h} \leq 11$ \\
\hline & $-14 \leq \mathrm{k} \leq 14$ & $-14 \leq \mathrm{k} \leq 11$ \\
\hline & $-16 \leq 1 \leq 14$ & $-15 \leq 1 \leq 15$ \\
\hline Reflections collected & 11099 & 9648 \\
\hline Independent reflections & $5742[\mathrm{R}$ (int) $=0.0259]$ & $5315[\mathrm{R}(\mathrm{int})=0.0379]$ \\
\hline Data / restraints / parameters & $5742 / 110 / 407$ & $5315 / 10 / 407$ \\
\hline Goodness-of-fit on $\mathrm{F}^{2}$ & 1.041 & 1.028 \\
\hline \multirow{2}{*}{ Final $R$ indices $[I>2 \sigma(I)]$} & $\mathrm{R} 1=0.0309$ & $\mathrm{R} 1=0.0336$ \\
\hline & $w R 2=0.0767$ & $\mathrm{wR} 2=0.0848$ \\
\hline \multirow{2}{*}{$\mathrm{R}$ indices (all data) } & $\mathrm{R} 1=0.0370$ & $\mathrm{R} 1=0.0373$ \\
\hline & $\mathrm{wR} 2=0.0813$ & $\mathrm{wR} 2=0.0878$ \\
\hline Largest diff. peak and hole $\left[\mathrm{e} \cdot \AA^{-3}\right]$ & 0.441 and -0.292 & 1.248 and -0.800 \\
\hline
\end{tabular}


Table S4. Data collection and structure refinement details for compounds 4, 5, 6 .

\begin{tabular}{|c|c|c|c|}
\hline & 4 & 6 & 5 \\
\hline CCDC No. & 1921067 & 1921068 & 1921069 \\
\hline Empirical formula & $\mathrm{C}_{37} \mathrm{H}_{30} \mathrm{NiOP}_{2} \mathrm{~S}$ & $\mathrm{C}_{66} \mathrm{H}_{62} \mathrm{~N}_{2} \mathrm{Ni}_{2} \mathrm{OP}_{4} \mathrm{~S}_{2}$ & $\mathrm{C}_{38} \mathrm{H}_{33} \mathrm{NNiP}_{2} \mathrm{~S}$ \\
\hline Molecular weight $\left[\mathrm{g} \cdot \mathrm{mol}^{-1}\right]$ & 643.32 & 1204.59 & 656.36 \\
\hline Temperature $[\mathrm{K}]$ & $100(2)$ & $100(2)$ & $100(2)$ \\
\hline Wavelength $[\AA]$ & 1.54184 & 1.54184 & 1.54184 \\
\hline Crystal system & Monoclinic & Monoclinic & Monoclinic \\
\hline Space group & $I 2 / \mathrm{a}$ & $P 2_{1} / \mathrm{n}$ & $P 2_{1} / \mathrm{c}$ \\
\hline $\mathrm{a}[\AA]$ & $18.10390(10)$ & $17.67220(10)$ & $14.56220(10)$ \\
\hline $\mathrm{b}[\AA]$ & $10.32090(10)$ & $16.98990(10)$ & $16.00050(10)$ \\
\hline $\mathrm{c}[\AA]$ & $33.2708(2)$ & $19.02000(10)$ & $15.23370(10)$ \\
\hline$\alpha\left[^{\circ}\right]$ & 90 & 90 & 90 \\
\hline$\beta\left[^{\circ}\right]$ & $98.3980(10)$ & $96.4720(10)$ & $118.2880(10)$ \\
\hline$\gamma\left[^{\circ}\right]$ & 90 & 90 & 90 \\
\hline Volume $\left[\AA^{3}\right]$ & $6149.94(8)$ & $5674.34(6)$ & $3125.60(4)$ \\
\hline Z & 8 & 4 & 4 \\
\hline Density (calculated) $\left[\mathrm{Mg} \cdot \mathrm{m}^{3}\right]$ & 1.390 & 1.410 & 1.395 \\
\hline Absorption coefficient $\left[\mathrm{mm}^{-1}\right]$ & 2.750 & 2.933 & 2.700 \\
\hline $\mathrm{F}(000)$ & 2672 & 2512 & 1368 \\
\hline Crystal size $[\mathrm{mm}]$ & $0.236 \times 0.153 \times 0.017$ & $0.332 \times 0.119 \times 0.042$ & $0.067 \times 0.059 \times 0.029$ \\
\hline$\theta$ range for data collection $\left[{ }^{\circ}\right]$ & 3.425 to 67.078 & 3.237 to 67.080 & 3.447 to 67.078 \\
\hline \multirow{3}{*}{ Index ranges } & $-21 \leq \mathrm{h} \leq 21$ & $-21 \leq \mathrm{h} \leq 21$ & $-17 \leq \mathrm{h} \leq 15$ \\
\hline & $-12 \leq \mathrm{k} \leq 12$ & $-20 \leq \mathrm{k} \leq 19$ & $-19 \leq \mathrm{k} \leq 19$ \\
\hline & $-25 \leq 1 \leq 39$ & $-22 \leq 1 \leq 22$ & $-18 \leq 1 \leq 17$ \\
\hline Reflections collected & 36796 & 78043 & 39795 \\
\hline Independent reflections & $5479[\mathrm{R}(\mathrm{int})=0.0253]$ & $10132[\mathrm{R}(\mathrm{int})=0.0434]$ & $5577[\mathrm{R}(\mathrm{int})=0.0329]$ \\
\hline Data / restraints / parameters & $5479 / 0 / 383$ & $10132 / 88 / 805$ & 5577 / 0 / 397 \\
\hline Goodness-of-fit on $\mathrm{F}^{2}$ & 1.027 & 1.381 & 1.024 \\
\hline Final $R$ indices $[\mathrm{I}>2 \sigma(\mathrm{I})]$ & $\begin{array}{c}\mathrm{R} 1=0.0251 \\
\mathrm{wR} 2=0.0677\end{array}$ & $\begin{array}{c}\mathrm{R} 1=0.0624 \\
\mathrm{wR} 2=0.1529\end{array}$ & $\begin{array}{c}\mathrm{R} 1=0.0264 \\
\mathrm{wR} 2=0.0726\end{array}$ \\
\hline $\mathrm{R}$ indices (all data) & $\begin{array}{c}\mathrm{R} 1=0.0263 \\
\mathrm{wR} 2=0.0686\end{array}$ & $\begin{array}{c}\mathrm{R} 1=0.0630 \\
\mathrm{wR} 2=0.1531\end{array}$ & $\begin{array}{c}\mathrm{R} 1=0.0287 \\
\mathrm{wR} 2=0.0741\end{array}$ \\
\hline Largest diff. peak and hole $\left[\mathrm{e} \cdot \AA^{-3}\right]$ & 0.450 and -0.240 & 0.442 and -0.431 & 0.362 and -0.231 \\
\hline
\end{tabular}




\subsection{Crystal Structure Determination of 1}

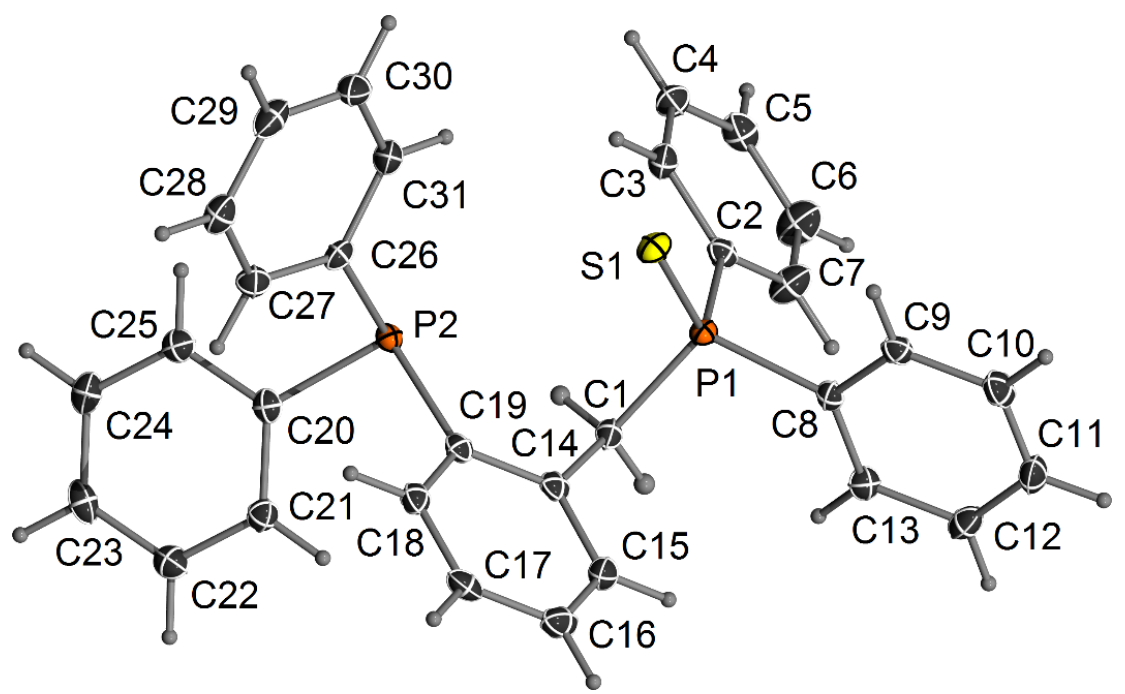

Figure S31 ORTEP Plot of compound 1. Ellipsoids are drawn at the 50\% probability level.

Table S5. Atomic coordinates $\left(\times 10^{4}\right)$ and equivalent isotropic displacement parameters $\left(\AA^{2} \times 10^{3}\right)$ for compound 40. $\mathrm{U}(\mathrm{eq})$ is defined as one third of the trace of the orthogonalized $\mathrm{U}_{\mathrm{ij}}$ tensor.

\begin{tabular}{|c|c|c|c|c|}
\hline & $\mathrm{x}$ & $\mathrm{y}$ & $\mathrm{Z}$ & $\mathrm{U}(\mathrm{eq})$ \\
\hline $\mathrm{P}(1)$ & $3380(1)$ & $3783(1)$ & $7185(1)$ & $11(1)$ \\
\hline $\mathrm{S}(1)$ & $3250(1)$ & 4583(1) & $8727(1)$ & $16(1)$ \\
\hline $\mathrm{C}(1)$ & 2714(1) & 2934(2) & $6526(2)$ & $14(1)$ \\
\hline $\mathrm{P}(2)$ & $1639(1)$ & 4481(1) & $7627(1)$ & $13(1)$ \\
\hline $\mathrm{C}(2)$ & $3553(1)$ & $5037(2)$ & $6080(1)$ & $14(1)$ \\
\hline $\mathrm{C}(4)$ & $3443(1)$ & $7310(2)$ & $5371(2)$ & $20(1)$ \\
\hline $\mathrm{C}(5)$ & $3756(1)$ & 6992(2) & 4382(2) & $21(1)$ \\
\hline $\mathrm{C}(6)$ & $3968(1)$ & $5701(2)$ & $4243(2)$ & $27(1)$ \\
\hline $\mathrm{C}(7)$ & $3867(1)$ & $4726(2)$ & $5085(2)$ & $23(1)$ \\
\hline $\mathrm{C}(8)$ & $4006(1)$ & 2612(2) & $7228(1)$ & $13(1)$ \\
\hline $\mathrm{C}(9)$ & $4459(1)$ & $2760(2)$ & $8118(2)$ & $15(1)$ \\
\hline$C(10)$ & $4976(1)$ & 1981(2) & $8111(2)$ & 21(1) \\
\hline $\mathrm{C}(11)$ & $5038(1)$ & $1038(2)$ & $7226(2)$ & $22(1)$ \\
\hline$C(12)$ & $4581(1)$ & $848(2)$ & $6365(2)$ & 19(1) \\
\hline$C(13)$ & $4068(1)$ & $1637(2)$ & $6357(2)$ & $16(1)$ \\
\hline$C(14)$ & $2375(1)$ & $2176(2)$ & 7432(1) & $14(1)$ \\
\hline$C(15)$ & $2545(1)$ & $856(2)$ & 7704(2) & $18(1)$ \\
\hline$C(16)$ & $2261(1)$ & $125(2)$ & $8554(2)$ & $20(1)$ \\
\hline $\mathrm{C}(17)$ & $1799(1)$ & $702(2)$ & $9150(2)$ & $18(1)$ \\
\hline $\mathrm{C}(18)$ & $1624(1)$ & 2011(2) & 8892(1) & $16(1)$ \\
\hline$C(19)$ & $1905(1)$ & $2768(2)$ & $8035(1)$ & $13(1)$ \\
\hline$C(20)$ & $868(1)$ & $4107(2)$ & 7066(1) & $14(1)$ \\
\hline$C(21)$ & $696(1)$ & $2851(2)$ & $6616(1)$ & $16(1)$ \\
\hline$C(22)$ & 119(1) & $2642(2)$ & $6125(2)$ & 19(1) \\
\hline$C(23)$ & $-297(1)$ & $3669(2)$ & $6081(2)$ & 19(1) \\
\hline$C(24)$ & $-132(1)$ & 4927(2) & $6512(2)$ & 19(1) \\
\hline$C(25)$ & $446(1)$ & $5146(2)$ & $6987(1)$ & $17(1)$ \\
\hline$C(26)$ & $1501(1)$ & $5286(2)$ & $9056(1)$ & $14(1)$ \\
\hline$C(27)$ & 1011(1) & $5003(2)$ & $9740(2)$ & $17(1)$ \\
\hline $\mathrm{C}(28)$ & $938(1)$ & $5668(2)$ & $10801(2)$ & $20(1)$ \\
\hline$C(29)$ & $1346(1)$ & $6635(2)$ & $11193(2)$ & $22(1)$ \\
\hline$C(30)$ & $1826(1)$ & $6955(2)$ & $10513(2)$ & $23(1)$ \\
\hline$C(31)$ & $1902(1)$ & $6285(2)$ & $9453(2)$ & $18(1)$ \\
\hline
\end{tabular}




\begin{tabular}{lllll}
\hline $\mathrm{C}(3)$ & $3341(1)$ & $6342(2)$ & $6218(2)$ & $17(1)$ \\
\hline
\end{tabular}

Table S6. Anisotropic displacement parameters $\left(\AA^{2} \times 10^{3}\right)$ for compound 1. The anisotropic displacement factor exponent takes the form: $-2 \pi^{2}\left[\mathrm{~h}^{2} \mathrm{a}^{* 2} \mathrm{U}^{11}+\ldots+2 \mathrm{~h} \mathrm{k} \mathrm{a}^{*} \mathrm{~b}^{*} \mathrm{U}^{12}\right]$.

\begin{tabular}{|c|c|c|c|c|c|c|}
\hline & $\mathrm{U}^{11}$ & $\mathrm{U}^{22}$ & $\mathrm{U}^{33}$ & $\mathrm{U}^{23}$ & $\mathrm{U}^{13}$ & $\mathrm{U}^{12}$ \\
\hline $\mathrm{P}(1)$ & $12(1)$ & $11(1)$ & 11(1) & $0(1)$ & $0(1)$ & 1(1) \\
\hline $\mathrm{S}(1)$ & $19(1)$ & $16(1)$ & $12(1)$ & $-3(1)$ & 1(1) & $2(1)$ \\
\hline $\mathrm{C}(1)$ & $12(1)$ & $16(1)$ & $13(1)$ & $-2(1)$ & $0(1)$ & $2(1)$ \\
\hline $\mathrm{P}(2)$ & $12(1)$ & $12(1)$ & $14(1)$ & $0(1)$ & $2(1)$ & $0(1)$ \\
\hline$C(2)$ & $13(1)$ & $14(1)$ & $14(1)$ & $2(1)$ & $-2(1)$ & $-1(1)$ \\
\hline $\mathrm{C}(4)$ & $19(1)$ & $15(1)$ & $26(1)$ & $4(1)$ & $-1(1)$ & $2(1)$ \\
\hline$C(5)$ & $20(1)$ & $22(1)$ & 21(1) & $9(1)$ & $0(1)$ & $-2(1)$ \\
\hline $\mathrm{C}(6)$ & $37(1)$ & $27(1)$ & $18(1)$ & $4(1)$ & 11(1) & $5(1)$ \\
\hline $\mathrm{C}(7)$ & $32(1)$ & $19(1)$ & $20(1)$ & $2(1)$ & $7(1)$ & $6(1)$ \\
\hline $\mathrm{C}(8)$ & $12(1)$ & $13(1)$ & $13(1)$ & $3(1)$ & $3(1)$ & $0(1)$ \\
\hline $\mathrm{C}(9)$ & $16(1)$ & $13(1)$ & $16(1)$ & $2(1)$ & $1(1)$ & $-2(1)$ \\
\hline$C(10)$ & $14(1)$ & $21(1)$ & $25(1)$ & $3(1)$ & $-5(1)$ & $-1(1)$ \\
\hline $\mathrm{C}(11)$ & $14(1)$ & $20(1)$ & $30(1)$ & $5(1)$ & $3(1)$ & $5(1)$ \\
\hline$C(12)$ & $21(1)$ & $16(1)$ & $20(1)$ & $0(1)$ & $7(1)$ & $4(1)$ \\
\hline$C(13)$ & $16(1)$ & $17(1)$ & $14(1)$ & $1(1)$ & $0(1)$ & $1(1)$ \\
\hline$C(14)$ & $12(1)$ & $15(1)$ & $14(1)$ & $-1(1)$ & $-3(1)$ & $-2(1)$ \\
\hline$C(15)$ & $14(1)$ & $17(1)$ & $22(1)$ & $-2(1)$ & $-1(1)$ & $2(1)$ \\
\hline$C(16)$ & $21(1)$ & $14(1)$ & $25(1)$ & $2(1)$ & $-5(1)$ & $0(1)$ \\
\hline$C(17)$ & $16(1)$ & $18(1)$ & $18(1)$ & $2(1)$ & $-3(1)$ & $-6(1)$ \\
\hline $\mathrm{C}(18)$ & $13(1)$ & $18(1)$ & $16(1)$ & $-2(1)$ & $-1(1)$ & $-1(1)$ \\
\hline$C(19)$ & $12(1)$ & $14(1)$ & $14(1)$ & $-1(1)$ & $-2(1)$ & $-2(1)$ \\
\hline$C(20)$ & $14(1)$ & $17(1)$ & 11(1) & $2(1)$ & $3(1)$ & $0(1)$ \\
\hline $\mathrm{C}(21)$ & $16(1)$ & $16(1)$ & $17(1)$ & $1(1)$ & $2(1)$ & 1(1) \\
\hline$C(22)$ & $19(1)$ & $20(1)$ & $17(1)$ & $-1(1)$ & $2(1)$ & $-3(1)$ \\
\hline$C(23)$ & $13(1)$ & $30(1)$ & $14(1)$ & $3(1)$ & $1(1)$ & $-2(1)$ \\
\hline$C(24)$ & $17(1)$ & $23(1)$ & $16(1)$ & $3(1)$ & $2(1)$ & $5(1)$ \\
\hline$C(25)$ & $19(1)$ & $16(1)$ & $16(1)$ & $0(1)$ & $3(1)$ & $0(1)$ \\
\hline$C(26)$ & $15(1)$ & $13(1)$ & $15(1)$ & $1(1)$ & $0(1)$ & $4(1)$ \\
\hline$C(27)$ & $18(1)$ & $15(1)$ & $18(1)$ & $2(1)$ & 1(1) & $0(1)$ \\
\hline$C(28)$ & $21(1)$ & $20(1)$ & $18(1)$ & $3(1)$ & $4(1)$ & $6(1)$ \\
\hline$C(29)$ & $25(1)$ & $22(1)$ & $18(1)$ & $-4(1)$ & $-2(1)$ & $10(1)$ \\
\hline$C(30)$ & $19(1)$ & $19(1)$ & $30(1)$ & $-8(1)$ & $-4(1)$ & $2(1)$ \\
\hline$C(31)$ & $14(1)$ & $17(1)$ & $24(1)$ & $-2(1)$ & $3(1)$ & $2(1)$ \\
\hline$C(3)$ & $14(1)$ & $18(1)$ & $18(1)$ & 1(1) & $2(1)$ & $2(1)$ \\
\hline
\end{tabular}




\subsection{Crystal Structure Determination of 1-Cl}

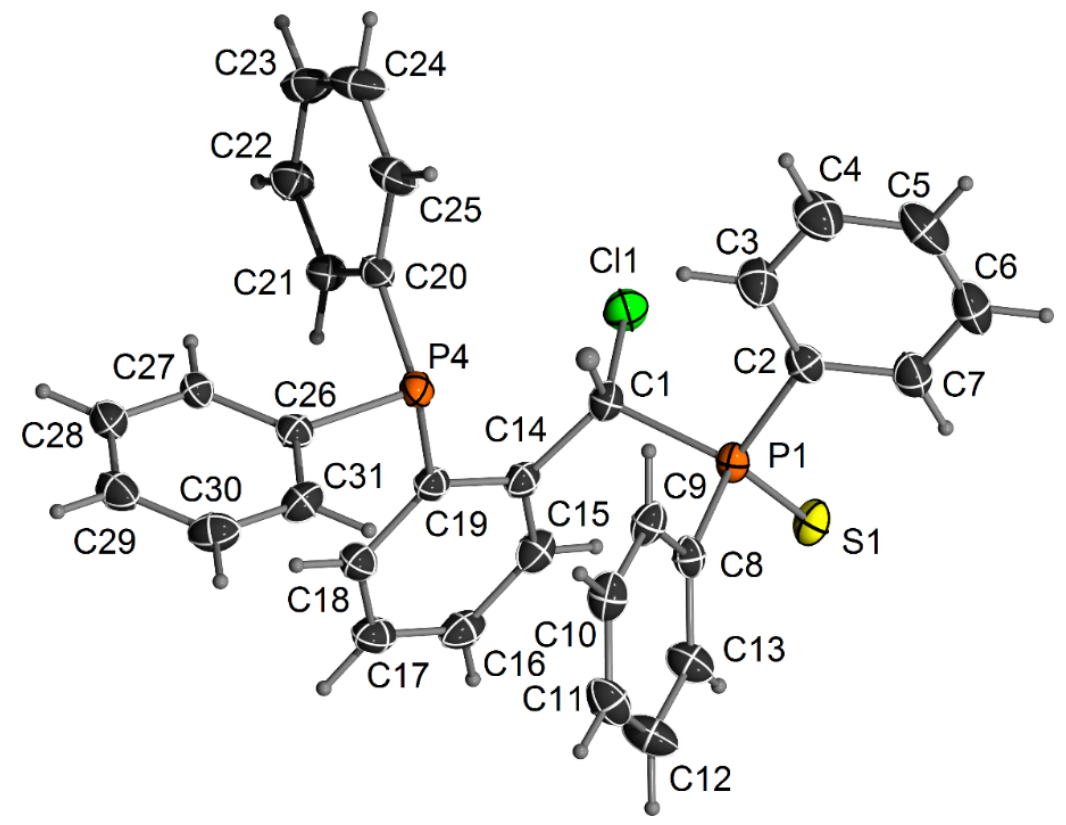

Figure S32. ORTEP Plot of compound 1-Cl. Ellipsoids are drawn at the 50\% probability level.

Table S7 Atomic coordinates ( $\left.\times 10^{4}\right)$ and equivalent isotropic displacement parameters $\left(\AA^{2} \times 10^{3}\right)$ for compound 1-Cl. $U(e q)$ is defined as one third of the trace of the orthogonalized $U_{i j}$ tensor.

\begin{tabular}{|c|c|c|c|c|}
\hline & $\mathrm{x}$ & $\mathrm{y}$ & $\mathrm{Z}$ & $\mathrm{U}(\mathrm{eq})$ \\
\hline $\mathrm{S}(1)$ & 8434(1) & $5487(1)$ & $8360(1)$ & $26(1)$ \\
\hline $\mathrm{P}(1)$ & $10092(1)$ & $4837(1)$ & 7947(1) & $20(1)$ \\
\hline $\mathrm{Cl}(1)$ & 8052(1) & 4142(1) & $6907(1)$ & $33(1)$ \\
\hline $\mathrm{C}(1)$ & $9868(3)$ & $4645(2)$ & $7069(1)$ & $23(1)$ \\
\hline $\mathrm{C}(2)$ & $10441(2)$ & $3727(2)$ & $8296(1)$ & $24(1)$ \\
\hline $\mathrm{C}(3)$ & $11109(3)$ & $3006(2)$ & 7962(1) & $35(1)$ \\
\hline $\mathrm{P}(4)$ & $12538(1)$ & 4497(1) & $6127(1)$ & $22(1)$ \\
\hline $\mathrm{C}(6)$ & $10480(4)$ & $2819(2)$ & 9261(1) & $41(1)$ \\
\hline$C(7)$ & 10107(3) & $3626(2)$ & $8945(1)$ & $32(1)$ \\
\hline $\mathrm{C}(8)$ & $11898(2)$ & $5429(2)$ & 7963(1) & $22(1)$ \\
\hline $\mathrm{C}(9)$ & $13236(3)$ & 4981(2) & $7792(1)$ & $25(1)$ \\
\hline $\mathrm{C}(10)$ & $14587(3)$ & $5464(2)$ & $7759(1)$ & $29(1)$ \\
\hline $\mathrm{C}(11)$ & 14612(3) & $6386(2)$ & 7901(1) & $33(1)$ \\
\hline $\mathrm{C}(12)$ & 13291(3) & $6830(2)$ & $8082(2)$ & $37(1)$ \\
\hline$C(13)$ & $11929(3)$ & $6353(2)$ & 8111(1) & $30(1)$ \\
\hline $\mathrm{C}(14)$ & $10138(3)$ & $5492(2)$ & $6676(1)$ & $22(1)$ \\
\hline$C(15)$ & $9232(3)$ & $6258(2)$ & $6768(1)$ & $27(1)$ \\
\hline$C(16)$ & $9544(3)$ & $7064(2)$ & $6444(1)$ & $30(1)$ \\
\hline$C(17)$ & $10738(3)$ & $7098(2)$ & $6014(1)$ & $30(1)$ \\
\hline$C(18)$ & 11619(3) & $6329(2)$ & $5903(1)$ & $26(1)$ \\
\hline$C(19)$ & 11343(3) & $5516(1)$ & $6232(1)$ & $22(1)$ \\
\hline$C(20)$ & $11318(3)$ & 3791(1) & $5620(1)$ & $24(1)$ \\
\hline$C(21)$ & 10203(3) & $4130(2)$ & $5212(1)$ & $27(1)$ \\
\hline$C(22)$ & $9410(3)$ & $3555(2)$ & $4807(1)$ & $33(1)$ \\
\hline$C(23)$ & $9727(3)$ & $2624(2)$ & $4806(2)$ & $38(1)$ \\
\hline$C(24)$ & 10811(3) & $2279(2)$ & $5220(2)$ & $38(1)$ \\
\hline$C(25)$ & $11602(3)$ & $2854(2)$ & $5628(2)$ & $32(1)$ \\
\hline$C(26)$ & 13995(3) & 4884(1) & $5561(1)$ & $22(1)$ \\
\hline$C(27)$ & $13876(3)$ & 4783(1) & 4894(1) & $23(1)$ \\
\hline$C(28)$ & $15078(3)$ & $5029(2)$ & $4500(1)$ & $29(1)$ \\
\hline
\end{tabular}




\begin{tabular}{lllll}
\hline $\mathrm{C}(29)$ & $16400(3)$ & $5385(2)$ & $4764(1)$ & $31(1)$ \\
$\mathrm{C}(30)$ & $16518(3)$ & $5491(2)$ & $5424(2)$ & $33(1)$ \\
$\mathrm{C}(31)$ & $15325(3)$ & $5238(2)$ & $5821(1)$ & $28(1)$ \\
$\mathrm{C}(5)$ & $11159(4)$ & $2114(2)$ & $8927(2)$ & $42(1)$ \\
$\mathrm{C}(4)$ & $11455(3)$ & $2203(2)$ & $8282(2)$ & $42(1)$ \\
\hline
\end{tabular}

Table S8. Anisotropic displacement parameters $\left(\AA^{2} \times 10^{3}\right)$ for compound 1-Cl. The anisotropic displacement factor exponent takes the form: $-2 \pi^{2}\left[\mathrm{~h}^{2} \mathrm{a}^{* 2} \mathrm{U}^{11}+\ldots+2 \mathrm{~h} \mathrm{k} \mathrm{a}^{*} \mathrm{~b}^{*} \mathrm{U}^{12}\right]$.

\begin{tabular}{|c|c|c|c|c|c|c|}
\hline & $\mathrm{U}^{11}$ & $\mathrm{U}^{22}$ & $\mathrm{U}^{33}$ & $\mathrm{U}^{23}$ & $\mathrm{U}^{13}$ & $\mathrm{U}^{12}$ \\
\hline $\mathrm{S}(1)$ & $19(1)$ & $35(1)$ & $25(1)$ & $-3(1)$ & $3(1)$ & $3(1)$ \\
\hline $\mathrm{P}(1)$ & $16(1)$ & $26(1)$ & $18(1)$ & 1(1) & $0(1)$ & $0(1)$ \\
\hline $\mathrm{Cl}(1)$ & $31(1)$ & $37(1)$ & $30(1)$ & $-2(1)$ & $-6(1)$ & $-8(1)$ \\
\hline $\mathrm{C}(1)$ & $22(1)$ & $26(1)$ & $20(1)$ & $-1(1)$ & 1(1) & 1(1) \\
\hline $\mathrm{C}(2)$ & $18(1)$ & $27(1)$ & $27(1)$ & $3(1)$ & $-1(1)$ & $-2(1)$ \\
\hline$C(3)$ & $39(1)$ & $35(1)$ & $31(1)$ & $5(1)$ & $5(1)$ & $9(1)$ \\
\hline $\mathrm{P}(4)$ & $28(1)$ & $19(1)$ & $20(1)$ & $3(1)$ & $2(1)$ & $3(1)$ \\
\hline$C(6)$ & $44(2)$ & $47(2)$ & $33(1)$ & $15(1)$ & $3(1)$ & $-5(1)$ \\
\hline$C(7)$ & $31(1)$ & $37(1)$ & $28(1)$ & $4(1)$ & 1(1) & $-5(1)$ \\
\hline $\mathrm{C}(8)$ & $19(1)$ & $27(1)$ & $19(1)$ & $3(1)$ & $-1(1)$ & $0(1)$ \\
\hline $\mathrm{C}(9)$ & $21(1)$ & $34(1)$ & $20(1)$ & $-3(1)$ & $-1(1)$ & 1(1) \\
\hline$C(10)$ & $17(1)$ & $43(1)$ & $26(1)$ & $0(1)$ & $-1(1)$ & $0(1)$ \\
\hline $\mathrm{C}(11)$ & $23(1)$ & $38(1)$ & $39(1)$ & $9(1)$ & $-2(1)$ & $-7(1)$ \\
\hline$C(12)$ & $29(1)$ & $25(1)$ & $57(2)$ & $7(1)$ & $-2(1)$ & $-4(1)$ \\
\hline$C(13)$ & $24(1)$ & $26(1)$ & $41(1)$ & $5(1)$ & $-1(1)$ & $1(1)$ \\
\hline$C(14)$ & $25(1)$ & $23(1)$ & $18(1)$ & $-2(1)$ & $-3(1)$ & $2(1)$ \\
\hline$C(15)$ & $28(1)$ & $31(1)$ & $23(1)$ & $-3(1)$ & $-3(1)$ & $7(1)$ \\
\hline$C(16)$ & $38(1)$ & $24(1)$ & $28(1)$ & $-4(1)$ & $-9(1)$ & $9(1)$ \\
\hline$C(17)$ & $38(1)$ & $20(1)$ & $31(1)$ & 1(1) & $-6(1)$ & $1(1)$ \\
\hline$C(18)$ & $31(1)$ & $22(1)$ & $25(1)$ & 1(1) & $-1(1)$ & $-1(1)$ \\
\hline$C(19)$ & $26(1)$ & $20(1)$ & $20(1)$ & $-1(1)$ & $-3(1)$ & $1(1)$ \\
\hline$C(20)$ & $27(1)$ & $18(1)$ & $27(1)$ & $1(1)$ & $9(1)$ & $-2(1)$ \\
\hline$C(21)$ & $32(1)$ & $21(1)$ & $28(1)$ & $-1(1)$ & $2(1)$ & $-2(1)$ \\
\hline$C(22)$ & $33(1)$ & $32(1)$ & $34(1)$ & $-2(1)$ & $2(1)$ & $-7(1)$ \\
\hline$C(23)$ & $34(1)$ & $30(1)$ & $50(2)$ & $-13(1)$ & 11(1) & $-13(1)$ \\
\hline$C(24)$ & $30(1)$ & $20(1)$ & $65(2)$ & $-7(1)$ & $10(1)$ & $-4(1)$ \\
\hline$C(25)$ & $27(1)$ & $20(1)$ & $48(2)$ & $3(1)$ & $9(1)$ & $0(1)$ \\
\hline$C(26)$ & $25(1)$ & $18(1)$ & $23(1)$ & 1(1) & $-1(1)$ & $3(1)$ \\
\hline$C(27)$ & $27(1)$ & $20(1)$ & $20(1)$ & $2(1)$ & $-1(1)$ & $-2(1)$ \\
\hline$C(28)$ & $36(1)$ & $24(1)$ & $26(1)$ & $3(1)$ & $3(1)$ & $0(1)$ \\
\hline$C(29)$ & $29(1)$ & $25(1)$ & $38(1)$ & $2(1)$ & $9(1)$ & $-1(1)$ \\
\hline$C(30)$ & $25(1)$ & $31(1)$ & $43(1)$ & $-7(1)$ & $-1(1)$ & $-2(1)$ \\
\hline$C(31)$ & $29(1)$ & $30(1)$ & $26(1)$ & $-5(1)$ & $-3(1)$ & $3(1)$ \\
\hline$C(5)$ & $39(2)$ & $37(1)$ & $52(2)$ & $20(1)$ & $0(1)$ & $-3(1)$ \\
\hline$C(4)$ & $44(2)$ & $32(1)$ & $49(2)$ & $7(1)$ & $6(1)$ & $9(1)$ \\
\hline
\end{tabular}




\subsection{Crystal Structure Determination of 1-NiCl}

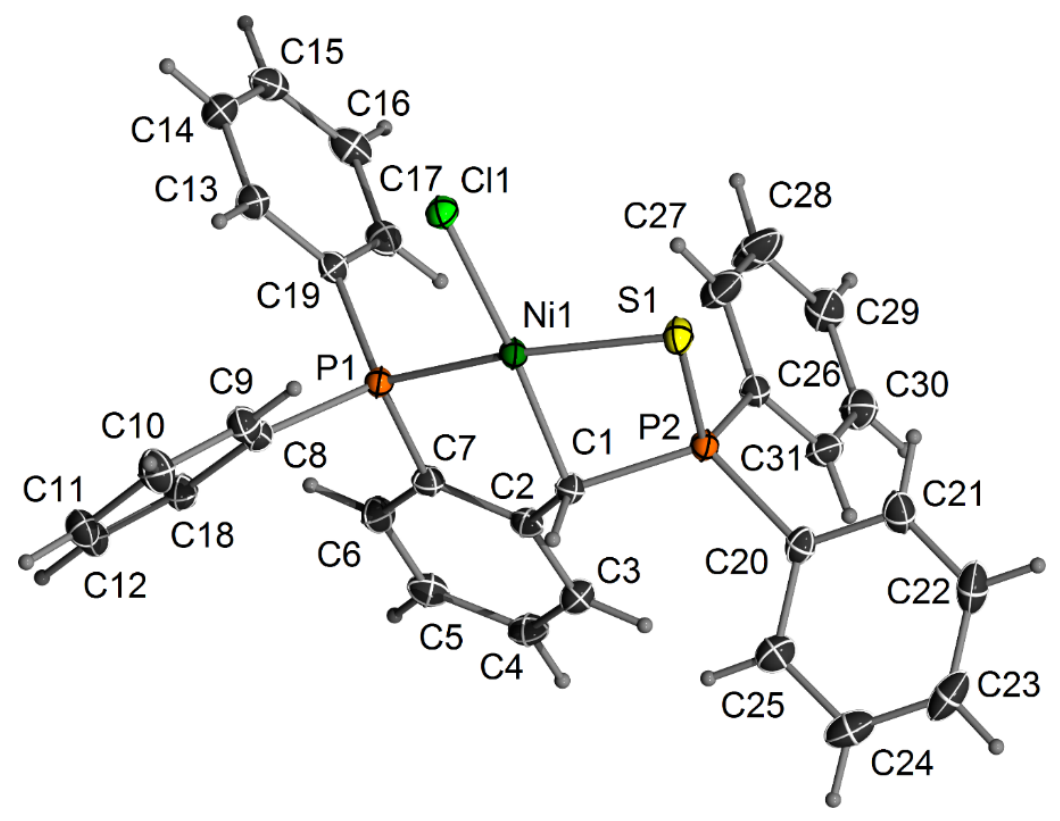

Figure S33. ORTEP Plot of compound 1-NiCl. Ellipsoids are drawn at the $50 \%$ probability level.

Table S9. Atomic coordinates ( $\left.\times 10^{4}\right)$ and equivalent isotropic displacement parameters $\left(\AA^{2} \times 10^{3}\right)$ for compound 1-NiCl. $\mathrm{U}(\mathrm{eq})$ is defined as one third of the trace of the orthogonalized $\mathrm{U}_{\mathrm{ij}}$ tensor.

\begin{tabular}{ccccc}
\hline & $\mathrm{x}$ & $\mathrm{y}$ & $\mathrm{Z}$ & $\mathrm{U}(\mathrm{eq})$ \\
\hline $\mathrm{Ni}(1)$ & $7393(1)$ & $4252(1)$ & $2647(1)$ & $14(1)$ \\
$\mathrm{Cl}(1)$ & $8336(1)$ & $4304(1)$ & $3607(1)$ & $18(1)$ \\
$\mathrm{S}(1)$ & $9085(1)$ & $3217(1)$ & $2337(1)$ & $19(1)$ \\
$\mathrm{P}(1)$ & $5794(1)$ & $5409(1)$ & $2736(1)$ & $14(1)$ \\
$\mathrm{P}(2)$ & $8014(1)$ & $3722(1)$ & $1554(1)$ & $14(1)$ \\
$\mathrm{C}(1)$ & $6419(1)$ & $4087(1)$ & $1813(1)$ & $15(1)$ \\
$\mathrm{C}(2)$ & $5535(1)$ & $5017(1)$ & $1552(1)$ & $16(1)$ \\
$\mathrm{C}(3)$ & $5102(1)$ & $5177(1)$ & $939(1)$ & $19(1)$ \\
$\mathrm{C}(4)$ & $4191(2)$ & $6015(1)$ & $739(1)$ & $20(1)$ \\
$\mathrm{C}(5)$ & $3691(1)$ & $6699(1)$ & $1150(1)$ & $20(1)$ \\
$\mathrm{C}(6)$ & $4135(1)$ & $6565(1)$ & $1762(1)$ & $19(1)$ \\
$\mathrm{C}(7)$ & $5061(1)$ & $5728(1)$ & $1963(1)$ & $16(1)$ \\
$\mathrm{C}(8)$ & $4349(1)$ & $4996(1)$ & $3124(1)$ & $17(1)$ \\
$\mathrm{C}(9)$ & $4543(2)$ & $4109(1)$ & $3504(1)$ & $21(1)$ \\
$\mathrm{C}(10)$ & $3435(2)$ & $3720(1)$ & $3777(1)$ & $25(1)$ \\
$\mathrm{C}(11)$ & $2131(2)$ & $4218(1)$ & $3672(1)$ & $25(1)$ \\
$\mathrm{C}(12)$ & $1939(2)$ & $5118(1)$ & $3303(1)$ & $23(1)$ \\
$\mathrm{C}(13)$ & $6456(1)$ & $6776(1)$ & $3720(1)$ & $20(1)$ \\
$\mathrm{C}(14)$ & $6990(2)$ & $7708(1)$ & $4008(1)$ & $23(1)$ \\
$\mathrm{C}(15)$ & $7496(1)$ & $8532(1)$ & $3680(1)$ & $23(1)$ \\
$\mathrm{C}(16)$ & $7487(2)$ & $8411(1)$ & $3066(1)$ & $23(1)$ \\
$\mathrm{C}(17)$ & $6960(1)$ & $7477(1)$ & $2774(1)$ & $20(1)$ \\
$\mathrm{C}(18)$ & $3039(2)$ & $5513(1)$ & $3032(1)$ & $20(1)$ \\
$\mathrm{C}(19)$ & $6417(1)$ & $6660(1)$ & $3099(1)$ & $17(1)$ \\
$\mathrm{C}(20)$ & $7903(1)$ & $2701(1)$ & $976(1)$ & $18(1)$ \\
$\mathrm{C}(21)$ & $9139(2)$ & $2197(1)$ & $864(1)$ & $24(1)$ \\
$\mathrm{C}(22)$ & $9071(2)$ & $1392(1)$ & $434(1)$ & $29(1)$ \\
$\mathrm{C}(23)$ & $7783(2)$ & $1082(1)$ & $118(1)$ & $30(1)$ \\
$\mathrm{C}(24)$ & $6555(2)$ & $1568(1)$ & $234(1)$ & $28(1)$ \\
$\mathrm{C}(25)$ & $6612(2)$ & $2380(1)$ & $663(1)$ & $22(1)$ \\
\hline
\end{tabular}




\begin{tabular}{lllll}
\hline $\mathrm{C}(26)$ & $8769(1)$ & $4890(1)$ & $1246(1)$ & $16(1)$ \\
$\mathrm{C}(27)$ & $9432(2)$ & $5678(1)$ & $1630(1)$ & $29(1)$ \\
$\mathrm{C}(28)$ & $9952(2)$ & $6591(1)$ & $1388(1)$ & $36(1)$ \\
$\mathrm{C}(29)$ & $9810(2)$ & $6739(1)$ & $769(1)$ & $26(1)$ \\
$\mathrm{C}(30)$ & $9166(2)$ & $5956(1)$ & $389(1)$ & $23(1)$ \\
$\mathrm{C}(31)$ & $8649(2)$ & $5027(1)$ & $625(1)$ & $21(1)$ \\
\hline
\end{tabular}

Table S10. Anisotropic displacement parameters $\left(\AA^{2} \times 10^{3}\right)$ for compound 1-NiCl. The anisotropic displacement factor exponent takes the form: $-2 \pi^{2}\left[\mathrm{~h}^{2} \mathrm{a}^{* 2} \mathrm{U}^{11}+\ldots+2 \mathrm{~h} \mathrm{k} \mathrm{a}^{*} \mathrm{~b}^{*} \mathrm{U}^{12}\right]$.

\begin{tabular}{|c|c|c|c|c|c|c|}
\hline & $\mathrm{U}^{11}$ & $\mathrm{U}^{22}$ & $\mathrm{U}^{33}$ & $\mathrm{U}^{23}$ & $\mathrm{U}^{13}$ & $\mathrm{U}^{12}$ \\
\hline $\mathrm{Ni}(1)$ & $13(1)$ & $17(1)$ & $12(1)$ & 1(1) & $3(1)$ & $2(1)$ \\
\hline $\mathrm{Cl}(1)$ & $17(1)$ & $25(1)$ & $13(1)$ & $0(1)$ & $2(1)$ & $3(1)$ \\
\hline $\mathrm{S}(1)$ & $17(1)$ & $24(1)$ & $15(1)$ & $2(1)$ & $4(1)$ & $7(1)$ \\
\hline $\mathrm{P}(1)$ & $14(1)$ & $16(1)$ & $13(1)$ & $0(1)$ & $3(1)$ & $2(1)$ \\
\hline $\mathrm{P}(2)$ & $15(1)$ & $16(1)$ & $13(1)$ & $0(1)$ & $4(1)$ & $1(1)$ \\
\hline $\mathrm{C}(1)$ & $15(1)$ & $17(1)$ & $14(1)$ & $0(1)$ & $3(1)$ & $0(1)$ \\
\hline $\mathrm{C}(2)$ & $14(1)$ & $16(1)$ & $17(1)$ & $1(1)$ & $2(1)$ & $-2(1)$ \\
\hline $\mathrm{C}(3)$ & $21(1)$ & $20(1)$ & $16(1)$ & $-1(1)$ & $2(1)$ & $0(1)$ \\
\hline $\mathrm{C}(4)$ & $22(1)$ & $23(1)$ & $16(1)$ & $2(1)$ & $-1(1)$ & $-1(1)$ \\
\hline $\mathrm{C}(5)$ & $18(1)$ & $19(1)$ & $22(1)$ & $3(1)$ & $-2(1)$ & $2(1)$ \\
\hline $\mathrm{C}(6)$ & $20(1)$ & $18(1)$ & $20(1)$ & $-1(1)$ & $2(1)$ & $2(1)$ \\
\hline$C(7)$ & $15(1)$ & $19(1)$ & $15(1)$ & 1(1) & 1(1) & $0(1)$ \\
\hline $\mathrm{C}(8)$ & $16(1)$ & $18(1)$ & $16(1)$ & $-4(1)$ & $4(1)$ & $-1(1)$ \\
\hline $\mathrm{C}(9)$ & $18(1)$ & $21(1)$ & $23(1)$ & $0(1)$ & $5(1)$ & 1(1) \\
\hline$C(10)$ & $25(1)$ & $25(1)$ & $28(1)$ & $3(1)$ & $9(1)$ & $-2(1)$ \\
\hline $\mathrm{C}(11)$ & $20(1)$ & $30(1)$ & $26(1)$ & $-5(1)$ & $10(1)$ & $-5(1)$ \\
\hline $\mathrm{C}(12)$ & $16(1)$ & $30(1)$ & $24(1)$ & $-7(1)$ & $4(1)$ & $2(1)$ \\
\hline $\mathrm{C}(13)$ & 19(1) & $22(1)$ & $19(1)$ & $-1(1)$ & $4(1)$ & $-2(1)$ \\
\hline$C(14)$ & $22(1)$ & $27(1)$ & $21(1)$ & $-6(1)$ & $3(1)$ & $-2(1)$ \\
\hline$C(15)$ & $17(1)$ & $19(1)$ & $33(1)$ & $-5(1)$ & $0(1)$ & $0(1)$ \\
\hline$C(16)$ & $18(1)$ & $19(1)$ & $30(1)$ & $7(1)$ & 1(1) & $0(1)$ \\
\hline $\mathrm{C}(17)$ & $18(1)$ & $23(1)$ & $19(1)$ & $4(1)$ & $2(1)$ & $2(1)$ \\
\hline $\mathrm{C}(18)$ & 19(1) & $22(1)$ & $20(1)$ & $-2(1)$ & $3(1)$ & $3(1)$ \\
\hline $\mathrm{C}(19)$ & $14(1)$ & $18(1)$ & $18(1)$ & $0(1)$ & $2(1)$ & $2(1)$ \\
\hline$C(20)$ & $23(1)$ & $15(1)$ & $16(1)$ & 1(1) & $6(1)$ & $-1(1)$ \\
\hline $\mathrm{C}(21)$ & $24(1)$ & $22(1)$ & $30(1)$ & $-3(1)$ & $12(1)$ & $-3(1)$ \\
\hline$C(22)$ & $38(1)$ & $22(1)$ & $32(1)$ & $-3(1)$ & $21(1)$ & 1(1) \\
\hline$C(23)$ & $54(1)$ & $20(1)$ & $17(1)$ & $-2(1)$ & $12(1)$ & $-3(1)$ \\
\hline$C(24)$ & $40(1)$ & $23(1)$ & $19(1)$ & $-1(1)$ & $-3(1)$ & $-4(1)$ \\
\hline$C(25)$ & $27(1)$ & $20(1)$ & $19(1)$ & 1(1) & 1(1) & 1(1) \\
\hline$C(26)$ & $15(1)$ & $17(1)$ & $17(1)$ & $0(1)$ & $4(1)$ & $0(1)$ \\
\hline$C(27)$ & $42(1)$ & $30(1)$ & $17(1)$ & $-5(1)$ & $7(1)$ & $-11(1)$ \\
\hline$C(28)$ & $55(1)$ & $29(1)$ & $26(1)$ & $-10(1)$ & $9(1)$ & $-20(1)$ \\
\hline $\mathrm{C}(29)$ & $33(1)$ & $20(1)$ & $28(1)$ & 1(1) & 11(1) & $-6(1)$ \\
\hline$C(30)$ & $26(1)$ & $25(1)$ & $18(1)$ & $3(1)$ & $5(1)$ & $-2(1)$ \\
\hline$C(31)$ & $23(1)$ & $21(1)$ & $17(1)$ & $-2(1)$ & 1(1) & $-4(1)$ \\
\hline
\end{tabular}




\subsection{Crystal Structure Determination of 1-PdCl}

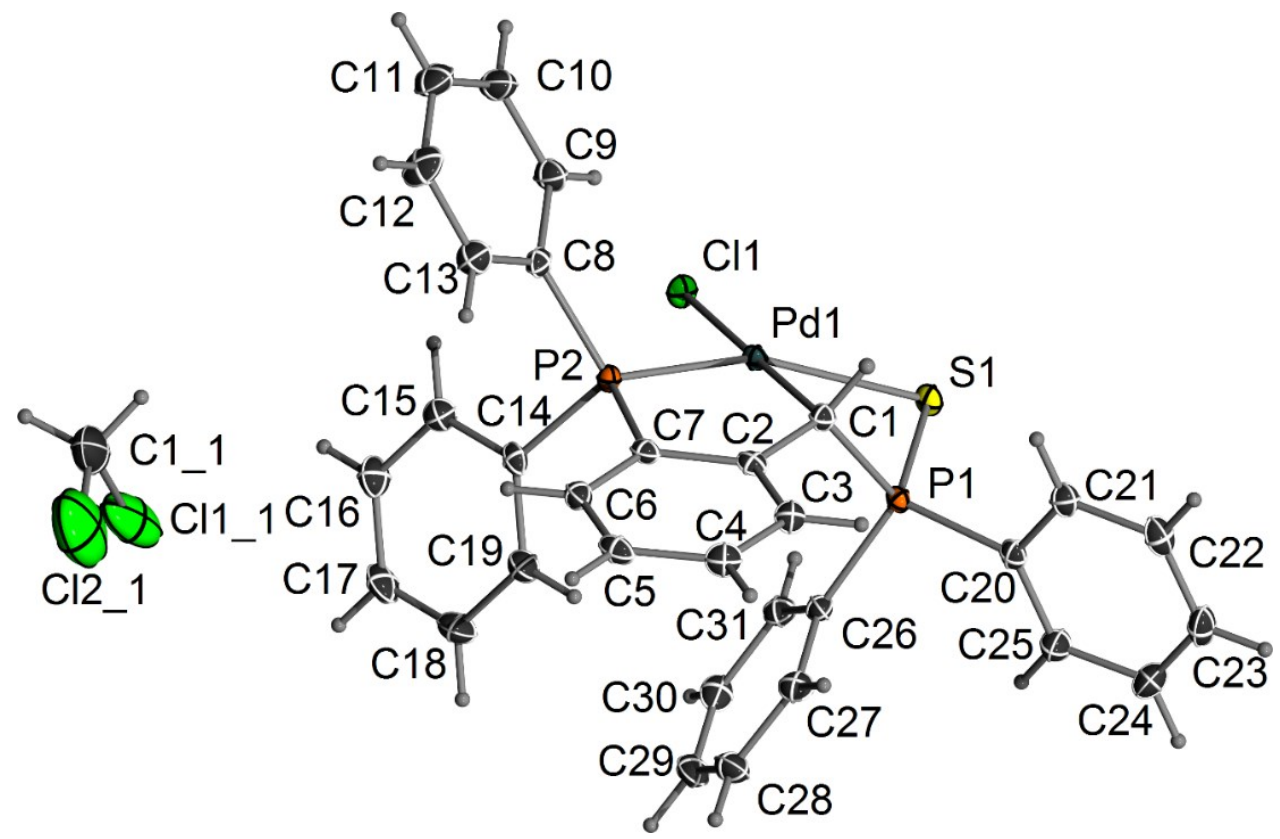

Figure S34. ORTEP Plot of compound 1-PdCl. Ellipsoids are drawn at the 50\% probability level.

Table S11. Atomic coordinates ( x 10 $)$ and equivalent isotropic displacement parameters $\left(\AA^{2} \times 10^{3}\right)$ for compound 1-PdCl. $\mathrm{U}(\mathrm{eq})$ is defined as one third of the trace of the orthogonalized $\mathrm{U}_{\mathrm{ij}}$ tensor.

\begin{tabular}{|c|c|c|c|c|}
\hline & $\mathrm{x}$ & $y$ & $\mathrm{Z}$ & $\mathrm{U}(\mathrm{eq})$ \\
\hline $\operatorname{Pd}(1)$ & $4072(1)$ & $6357(1)$ & $367(1)$ & $10(1)$ \\
\hline $\mathrm{Cl}(1)$ & $5407(1)$ & $6615(1)$ & $1618(1)$ & $15(1)$ \\
\hline $\mathrm{S}(1)$ & $5869(1)$ & $6717(1)$ & $-1346(1)$ & $14(1)$ \\
\hline $\mathrm{P}(1)$ & 4139(1) & $7231(1)$ & $-1897(1)$ & 11(1) \\
\hline $\mathrm{C}(1)$ & $2962(2)$ & $6158(2)$ & $-764(2)$ & $12(1)$ \\
\hline$C(2)$ & $1482(2)$ & $6392(2)$ & $-565(2)$ & $12(1)$ \\
\hline $\mathrm{P}(2)$ & $2042(1)$ & $6467(1)$ & $1380(1)$ & 11(1) \\
\hline$C(3)$ & $682(2)$ & $6434(2)$ & $-1358(2)$ & $15(1)$ \\
\hline$C(4)$ & $-681(2)$ & $6662(2)$ & $-1160(2)$ & $17(1)$ \\
\hline$C(6)$ & $-506(2)$ & $6806(2)$ & $630(2)$ & $15(1)$ \\
\hline$C(7)$ & $867(2)$ & $6559(2)$ & $440(2)$ & $12(1)$ \\
\hline $\mathrm{C}(8)$ & 1481(2) & $5258(2)$ & $2750(2)$ & $13(1)$ \\
\hline $\mathrm{C}(9)$ & $2473(2)$ & $4650(2)$ & $3321(2)$ & $19(1)$ \\
\hline$C(10)$ & $2107(2)$ & $3732(2)$ & $4379(2)$ & $24(1)$ \\
\hline $\mathrm{C}(11)$ & $746(2)$ & $3404(2)$ & $4866(2)$ & $25(1)$ \\
\hline$C(12)$ & $-249(2)$ & $3998(2)$ & $4296(2)$ & $25(1)$ \\
\hline$C(13)$ & $106(2)$ & $4927(2)$ & $3245(2)$ & $20(1)$ \\
\hline$C(14)$ & $1853(2)$ & $7824(2)$ & $1690(2)$ & $14(1)$ \\
\hline$C(15)$ & $2119(2)$ & $7851(2)$ & $2707(2)$ & $18(1)$ \\
\hline$C(16)$ & 2092(2) & $8915(2)$ & $2899(2)$ & $22(1)$ \\
\hline$C(17)$ & $1800(2)$ & $9958(2)$ & $2078(2)$ & $23(1)$ \\
\hline $\mathrm{C}(18)$ & $1530(3)$ & $9935(2)$ & $1068(2)$ & $26(1)$ \\
\hline$C(19)$ & $1563(2)$ & $8874(2)$ & $866(2)$ & $21(1)$ \\
\hline$C(20)$ & 4191(2) & $7253(2)$ & $-3325(2)$ & $14(1)$ \\
\hline $\mathrm{C}(21)$ & $3889(2)$ & $6200(2)$ & $-3466(2)$ & $16(1)$ \\
\hline$C(22)$ & $4061(2)$ & $6178(2)$ & $-4566(2)$ & 19(1) \\
\hline $\mathrm{C}(23)$ & $4534(2)$ & $7194(2)$ & $-5513(2)$ & $22(1)$ \\
\hline$C(24)$ & $4830(2)$ & $8245(2)$ & $-5370(2)$ & $24(1)$ \\
\hline
\end{tabular}




\begin{tabular}{lcccc}
\hline $\mathrm{C}(25)$ & $4661(2)$ & $8278(2)$ & $-4281(2)$ & $20(1)$ \\
$\mathrm{C}(26)$ & $3703(2)$ & $8712(2)$ & $-1939(2)$ & $14(1)$ \\
$\mathrm{C}(27)$ & $2563(2)$ & $9220(2)$ & $-2363(2)$ & $17(1)$ \\
$\mathrm{C}(28)$ & $2200(2)$ & $10338(2)$ & $-2345(2)$ & $21(1)$ \\
$\mathrm{C}(29)$ & $2984(2)$ & $10948(2)$ & $-1929(2)$ & $22(1)$ \\
$\mathrm{C}(30)$ & $4122(2)$ & $10454(2)$ & $-1519(2)$ & $20(1)$ \\
$\mathrm{C}(31)$ & $4482(2)$ & $9329(2)$ & $-1515(2)$ & $16(1)$ \\
$\mathrm{C}(5)$ & $-1283(2)$ & $6856(2)$ & $-162(2)$ & $16(1)$ \\
$\mathrm{C} 121$ & $-1080(4)$ & $9664(5)$ & $4970(5)$ & $81(1)$ \\
$\mathrm{C} 11$ & $1651(4)$ & $10543(3)$ & $4816(4)$ & $64(1)$ \\
$\mathrm{C} 11$ & $284(8)$ & $9334(7)$ & $5522(6)$ & $77(3)$ \\
\hline
\end{tabular}

Table S12. Anisotropic displacement parameters $\left(\AA^{2} \times 10^{3}\right)$ for compound 1-PdCl. The anisotropic displacement factor exponent takes the form: $-2 \pi^{2}\left[\mathrm{~h}^{2} \mathrm{a}^{* 2} \mathrm{U}^{11}+\ldots+2 \mathrm{~h} \mathrm{k} \mathrm{a}^{*} \mathrm{~b}^{*} \mathrm{U}^{12}\right]$.

\begin{tabular}{|c|c|c|c|c|c|c|}
\hline & $\mathrm{U}^{11}$ & $\mathrm{U}^{22}$ & $\mathrm{U}^{33}$ & $\mathrm{U}^{23}$ & $\mathrm{U}^{13}$ & $\mathrm{U}^{12}$ \\
\hline $\operatorname{Pd}(1)$ & $9(1)$ & $11(1)$ & $10(1)$ & $-4(1)$ & $-2(1)$ & 1(1) \\
\hline $\mathrm{Cl}(1)$ & $14(1)$ & $17(1)$ & $15(1)$ & $-7(1)$ & $-5(1)$ & $0(1)$ \\
\hline $\mathrm{S}(1)$ & $9(1)$ & $19(1)$ & $14(1)$ & $-7(1)$ & $-3(1)$ & $3(1)$ \\
\hline $\mathrm{P}(1)$ & $9(1)$ & $11(1)$ & $11(1)$ & $-4(1)$ & $-2(1)$ & 1(1) \\
\hline $\mathrm{C}(1)$ & 13(1) & $11(1)$ & $11(1)$ & $-4(1)$ & $-3(1)$ & $1(1)$ \\
\hline $\mathrm{C}(2)$ & 11(1) & $8(1)$ & $15(1)$ & $-4(1)$ & $-2(1)$ & $-1(1)$ \\
\hline $\mathrm{P}(2)$ & 10(1) & $11(1)$ & $11(1)$ & $-4(1)$ & $-1(1)$ & $1(1)$ \\
\hline $\mathrm{C}(3)$ & $15(1)$ & $15(1)$ & $16(1)$ & $-6(1)$ & $-3(1)$ & $-1(1)$ \\
\hline $\mathrm{C}(4)$ & $15(1)$ & $18(1)$ & $20(1)$ & $-7(1)$ & $-6(1)$ & $-1(1)$ \\
\hline $\mathrm{C}(6)$ & $15(1)$ & $12(1)$ & $16(1)$ & $-5(1)$ & $0(1)$ & $1(1)$ \\
\hline $\mathrm{C}(7)$ & 13(1) & $9(1)$ & $14(1)$ & $-3(1)$ & $-2(1)$ & $1(1)$ \\
\hline $\mathrm{C}(8)$ & 17(1) & $12(1)$ & $11(1)$ & $-6(1)$ & $-1(1)$ & $-1(1)$ \\
\hline $\mathrm{C}(9)$ & 14(1) & $19(1)$ & $20(1)$ & $-4(1)$ & $-1(1)$ & $3(1)$ \\
\hline$C(10)$ & $22(1)$ & $22(1)$ & $22(1)$ & $-1(1)$ & $-4(1)$ & $6(1)$ \\
\hline $\mathrm{C}(11)$ & $25(1)$ & $23(1)$ & $19(1)$ & 1(1) & $-1(1)$ & $-1(1)$ \\
\hline$C(12)$ & $17(1)$ & $29(1)$ & $22(1)$ & $-2(1)$ & $2(1)$ & $-5(1)$ \\
\hline$C(13)$ & $16(1)$ & $23(1)$ & $19(1)$ & $-5(1)$ & $-4(1)$ & $0(1)$ \\
\hline$C(14)$ & $10(1)$ & $15(1)$ & $16(1)$ & $-7(1)$ & 1(1) & $0(1)$ \\
\hline$C(15)$ & $20(1)$ & $17(1)$ & $17(1)$ & $-6(1)$ & $-3(1)$ & $1(1)$ \\
\hline$C(16)$ & $25(1)$ & $23(1)$ & $23(1)$ & $-14(1)$ & $-4(1)$ & $1(1)$ \\
\hline $\mathrm{C}(17)$ & $26(1)$ & $16(1)$ & $30(1)$ & $-14(1)$ & $-1(1)$ & $1(1)$ \\
\hline $\mathrm{C}(18)$ & $36(1)$ & $15(1)$ & $27(1)$ & $-6(1)$ & $-7(1)$ & $6(1)$ \\
\hline$C(19)$ & $28(1)$ & $16(1)$ & $19(1)$ & $-7(1)$ & $-4(1)$ & $4(1)$ \\
\hline$C(20)$ & $10(1)$ & $18(1)$ & $14(1)$ & $-7(1)$ & $-3(1)$ & $4(1)$ \\
\hline$C(21)$ & $14(1)$ & $18(1)$ & $17(1)$ & $-7(1)$ & $-2(1)$ & $1(1)$ \\
\hline$C(22)$ & $16(1)$ & $25(1)$ & $24(1)$ & $-15(1)$ & $-7(1)$ & $5(1)$ \\
\hline$C(23)$ & 19(1) & $34(1)$ & $16(1)$ & $-13(1)$ & $-5(1)$ & $7(1)$ \\
\hline$C(24)$ & $29(1)$ & $24(1)$ & $14(1)$ & $-4(1)$ & $-2(1)$ & $2(1)$ \\
\hline$C(25)$ & $25(1)$ & $18(1)$ & $16(1)$ & $-6(1)$ & $-2(1)$ & 1(1) \\
\hline$C(26)$ & $14(1)$ & 11(1) & $13(1)$ & $-3(1)$ & $1(1)$ & $0(1)$ \\
\hline$C(27)$ & $16(1)$ & $15(1)$ & $18(1)$ & $-5(1)$ & $-3(1)$ & $-1(1)$ \\
\hline $\mathrm{C}(28)$ & $19(1)$ & $17(1)$ & $25(1)$ & $-6(1)$ & $-3(1)$ & $5(1)$ \\
\hline$C(29)$ & $26(1)$ & $12(1)$ & $23(1)$ & $-7(1)$ & $2(1)$ & 1(1) \\
\hline$C(30)$ & $25(1)$ & $17(1)$ & $19(1)$ & $-8(1)$ & $0(1)$ & $-5(1)$ \\
\hline$C(31)$ & $17(1)$ & $15(1)$ & $15(1)$ & $-4(1)$ & $-2(1)$ & $-1(1)$ \\
\hline $\mathrm{C}(5)$ & 11(1) & $13(1)$ & $23(1)$ & $-5(1)$ & $-2(1)$ & $1(1)$ \\
\hline $\mathrm{Cl} 21$ & $92(3)$ & $100(3)$ & $88(2)$ & $-71(2)$ & $-33(2)$ & $28(2)$ \\
\hline Cl11 & $99(3)$ & $57(2)$ & $72(2)$ & $-49(1)$ & $-56(2)$ & $39(2)$ \\
\hline C11 & $134(9)$ & $71(5)$ & $31(3)$ & $-26(3)$ & $-19(4)$ & $63(6)$ \\
\hline
\end{tabular}




\subsection{Crystal Structure Determination of 1-Ni}

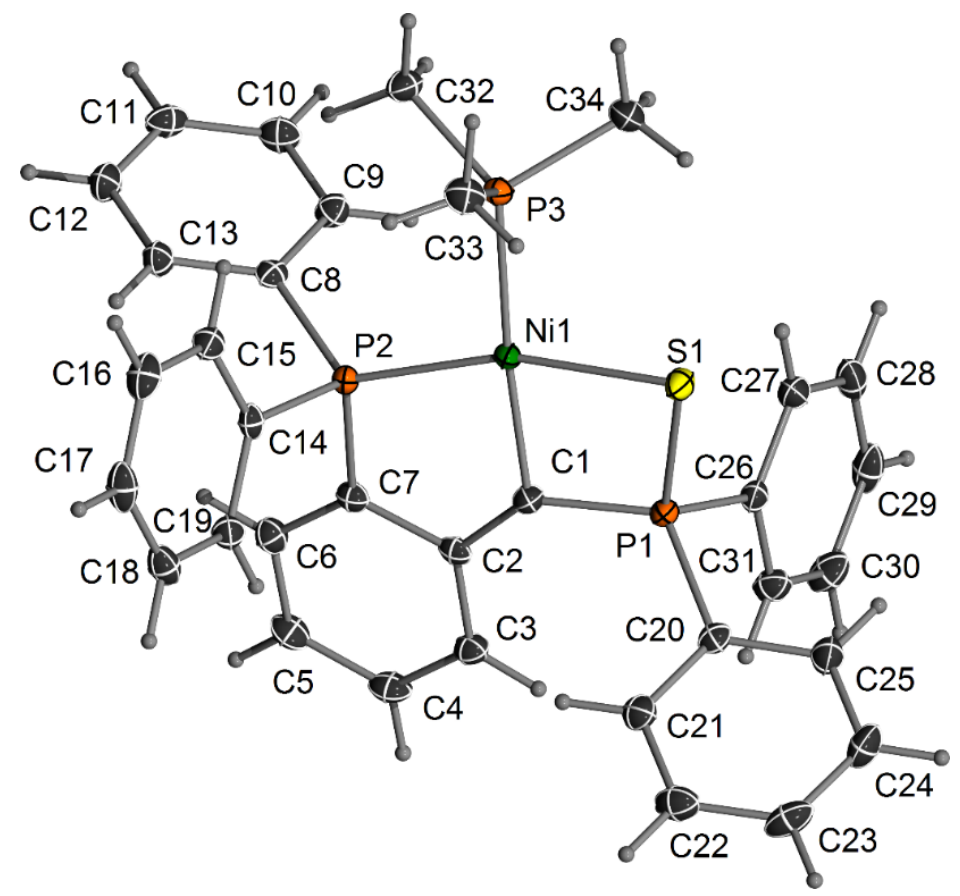

Figure S35. ORTEP Plot of compound 1-Ni. Ellipsoids are drawn at the 50\% probability level.

Table S13. Atomic coordinates $\left(\times 10^{4}\right)$ and equivalent isotropic displacement parameters $\left(\AA^{2} \times 10^{3}\right)$ for compound 1-Ni. $U(\mathrm{eq})$ is defined as one third of the trace of the orthogonalized $U_{\mathrm{ij}}$ tensor.

\begin{tabular}{ccccc}
\hline & $\mathrm{X}$ & $\mathrm{y}$ & $\mathrm{z}$ & $\mathrm{U}(\mathrm{eq})$ \\
\hline $\mathrm{Ni}(1)$ & $2169(1)$ & $1805(1)$ & $5666(1)$ & $12(1)$ \\
$\mathrm{S}(1)$ & $147(1)$ & $1879(1)$ & $4780(1)$ & $18(1)$ \\
$\mathrm{P}(1)$ & $518(1)$ & $2896(1)$ & $5259(1)$ & $13(1)$ \\
$\mathrm{C}(1)$ & $1924(2)$ & $2754(1)$ & $6066(1)$ & $15(1)$ \\
$\mathrm{C}(21)$ & $-546(2)$ & $3243(1)$ & $6671(1)$ & $19(1)$ \\
$\mathrm{C}(20)$ & $-709(2)$ & $3230(1)$ & $5681(1)$ & $15(1)$ \\
$\mathrm{C}(2)$ & $2862(2)$ & $3207(1)$ & $6642(1)$ & $13(1)$ \\
$\mathrm{P}(2)$ & $3914(1)$ & $1954(1)$ & $6748(1)$ & $12(1)$ \\
$\mathrm{C}(22)$ & $-1528(2)$ & $3427(1)$ & $6998(1)$ & $23(1)$ \\
$\mathrm{C}(3)$ & $2777(2)$ & $3938(1)$ & $6862(1)$ & $17(1)$ \\
$\mathrm{C}(23)$ & $-2654(2)$ & $3599(1)$ & $6338(2)$ & $25(1)$ \\
$\mathrm{C}(4)$ & $3797(2)$ & $4301(1)$ & $7438(1)$ & $20(1)$ \\
$\mathrm{C}(25)$ & $-1844(2)$ & $3414(1)$ & $5022(1)$ & $21(1)$ \\
$\mathrm{C}(6)$ & $5049(2)$ & $3252(1)$ & $7627(1)$ & $18(1)$ \\
$\mathrm{C}(26)$ & $487(2)$ & $3494(1)$ & $4269(1)$ & $16(1)$ \\
$\mathrm{C}(7)$ & $4027(2)$ & $2886(1)$ & $7049(1)$ & $14(1)$ \\
$\mathrm{C}(27)$ & $645(2)$ & $3238(1)$ & $3416(1)$ & $20(1)$ \\
$\mathrm{C}(8)$ & $5362(2)$ & $1728(1)$ & $6549(1)$ & $14(1)$ \\
$\mathrm{C}(28)$ & $774(2)$ & $3706(1)$ & $2716(1)$ & $24(1)$ \\
$\mathrm{C}(9)$ & $5478(2)$ & $1879(1)$ & $5640(1)$ & $18(1)$ \\
$\mathrm{C}(29)$ & $762(2)$ & $4433(1)$ & $2870(1)$ & $26(1)$ \\
$\mathrm{C}(10)$ & $6589(2)$ & $1774(1)$ & $5470(1)$ & $21(1)$ \\
$\mathrm{C}(11)$ & $7594(2)$ & $1514(1)$ & $6200(1)$ & $20(1)$ \\
$\mathrm{C}(14)$ & $3952(2)$ & $1498(1)$ & $7866(1)$ & $14(1)$ \\
$\mathrm{C}(15)$ & $4139(2)$ & $764(1)$ & $7957(1)$ & $17(1)$ \\
$\mathrm{C}(16)$ & $4070(2)$ & $412(1)$ & $8777(1)$ & $22(1)$ \\
$\mathrm{C}(17)$ & $3807(2)$ & $782(1)$ & $9512(1)$ & $22(1)$ \\
$\mathrm{C}(18)$ & $3625(2)$ & $1511(1)$ & $9431(1)$ & $20(1)$ \\
\hline & & & &
\end{tabular}




\begin{tabular}{ccccc}
\hline $\mathrm{C}(13)$ & $6375(2)$ & $1461(1)$ & $7276(1)$ & $18(1)$ \\
$\mathrm{C}(12)$ & $7487(2)$ & $1353(1)$ & $7101(1)$ & $22(1)$ \\
$\mathrm{C}(19)$ & $3701(2)$ & $1868(1)$ & $8612(1)$ & $16(1)$ \\
$\mathrm{C}(30)$ & $608(2)$ & $4695(1)$ & $3719(1)$ & $24(1)$ \\
$\mathrm{C}(32)$ & $3788(2)$ & $357(1)$ & $5118(1)$ & $19(1)$ \\
$\mathrm{C}(31)$ & $469(2)$ & $4226(1)$ & $4414(1)$ & $20(1)$ \\
$\mathrm{C}(33)$ & $1717(2)$ & $42(1)$ & $5713(1)$ & $21(1)$ \\
$\mathrm{P}(3)$ & $2321(1)$ & $732(1)$ & $5109(1)$ & $14(1)$ \\
$\mathrm{C}(34)$ & $1436(2)$ & $604(1)$ & $3841(1)$ & $22(1)$ \\
$\mathrm{C}(24)$ & $-2815(2)$ & $3599(1)$ & $5348(2)$ & $24(1)$ \\
$\mathrm{C}(5)$ & $4936(2)$ & $3968(1)$ & $7825(1)$ & $21(1)$ \\
\hline
\end{tabular}

Table S14. Anisotropic displacement parameters $\left(\AA^{2} \times 10^{3}\right)$ for compound 1-Ni. The anisotropic displacement factor exponent takes the form: $-2 \pi^{2}\left[\mathrm{~h}^{2} \mathrm{a}^{* 2} \mathrm{U}^{11}+\ldots+2 \mathrm{~h} \mathrm{k} \mathrm{a}^{*} \mathrm{~b}^{*} \mathrm{U}^{12}\right]$.

\begin{tabular}{|c|c|c|c|c|c|c|}
\hline & $\mathrm{U}^{11}$ & $\mathrm{U}^{22}$ & $\mathrm{U}^{33}$ & $\mathrm{U}^{23}$ & $\mathrm{U}^{13}$ & $\mathrm{U}^{12}$ \\
\hline $\mathrm{Ni}(1)$ & 11(1) & 11(1) & $12(1)$ & $-2(1)$ & $2(1)$ & $1(1)$ \\
\hline $\mathrm{S}(1)$ & $13(1)$ & $14(1)$ & $22(1)$ & $-4(1)$ & $0(1)$ & 1(1) \\
\hline $\mathrm{P}(1)$ & $13(1)$ & $12(1)$ & $14(1)$ & $-1(1)$ & $3(1)$ & $2(1)$ \\
\hline $\mathrm{C}(1)$ & $15(1)$ & $13(1)$ & $15(1)$ & $-1(1)$ & $4(1)$ & $2(1)$ \\
\hline $\mathrm{C}(21)$ & $22(1)$ & $16(1)$ & $18(1)$ & $2(1)$ & $6(1)$ & $2(1)$ \\
\hline $\mathrm{C}(20)$ & $16(1)$ & $12(1)$ & $17(1)$ & $2(1)$ & $6(1)$ & 1(1) \\
\hline $\mathrm{C}(2)$ & $17(1)$ & $13(1)$ & $10(1)$ & 1(1) & $6(1)$ & $-1(1)$ \\
\hline $\mathrm{P}(2)$ & 11(1) & $12(1)$ & $11(1)$ & $0(1)$ & $2(1)$ & $1(1)$ \\
\hline$C(22)$ & $32(1)$ & $21(1)$ & $21(1)$ & $2(1)$ & $14(1)$ & $3(1)$ \\
\hline $\mathrm{C}(3)$ & 21(1) & $13(1)$ & $17(1)$ & $0(1)$ & $6(1)$ & $1(1)$ \\
\hline$C(23)$ & $27(1)$ & $21(1)$ & $36(1)$ & $5(1)$ & $21(1)$ & $6(1)$ \\
\hline $\mathrm{C}(4)$ & $27(1)$ & $13(1)$ & $21(1)$ & $-3(1)$ & $10(1)$ & $-3(1)$ \\
\hline$C(25)$ & $18(1)$ & $25(1)$ & $18(1)$ & $3(1)$ & $4(1)$ & $4(1)$ \\
\hline $\mathrm{C}(6)$ & $16(1)$ & $19(1)$ & $17(1)$ & $-1(1)$ & $5(1)$ & $-2(1)$ \\
\hline$C(26)$ & $13(1)$ & $19(1)$ & $14(1)$ & $2(1)$ & $3(1)$ & $4(1)$ \\
\hline $\mathrm{C}(7)$ & $17(1)$ & $13(1)$ & $13(1)$ & $1(1)$ & $6(1)$ & $-1(1)$ \\
\hline$C(27)$ & $16(1)$ & $26(1)$ & $17(1)$ & $-3(1)$ & $4(1)$ & $4(1)$ \\
\hline $\mathrm{C}(8)$ & $14(1)$ & $10(1)$ & $17(1)$ & $-3(1)$ & $4(1)$ & $-2(1)$ \\
\hline$C(28)$ & $16(1)$ & $43(1)$ & $14(1)$ & $1(1)$ & $4(1)$ & $5(1)$ \\
\hline $\mathrm{C}(9)$ & $18(1)$ & $18(1)$ & $18(1)$ & $2(1)$ & $6(1)$ & $2(1)$ \\
\hline$C(29)$ & $18(1)$ & $40(1)$ & $20(1)$ & $14(1)$ & $9(1)$ & $7(1)$ \\
\hline$C(10)$ & $24(1)$ & $22(1)$ & $20(1)$ & $1(1)$ & $12(1)$ & $-1(1)$ \\
\hline $\mathrm{C}(11)$ & $17(1)$ & $21(1)$ & $26(1)$ & $-5(1)$ & $11(1)$ & $-2(1)$ \\
\hline$C(14)$ & $10(1)$ & $18(1)$ & $13(1)$ & $2(1)$ & 1(1) & $-1(1)$ \\
\hline$C(15)$ & $16(1)$ & $15(1)$ & $19(1)$ & $-1(1)$ & $3(1)$ & $0(1)$ \\
\hline$C(16)$ & 19(1) & $18(1)$ & $26(1)$ & $7(1)$ & $3(1)$ & $-2(1)$ \\
\hline $\mathrm{C}(17)$ & $17(1)$ & $31(1)$ & $18(1)$ & $9(1)$ & $3(1)$ & $-3(1)$ \\
\hline $\mathrm{C}(18)$ & $17(1)$ & $30(1)$ & $14(1)$ & $0(1)$ & $5(1)$ & $-3(1)$ \\
\hline $\mathrm{C}(13)$ & $13(1)$ & $24(1)$ & $15(1)$ & $-1(1)$ & $3(1)$ & $0(1)$ \\
\hline$C(12)$ & $14(1)$ & $29(1)$ & $21(1)$ & $-2(1)$ & $2(1)$ & $2(1)$ \\
\hline$C(19)$ & $16(1)$ & $17(1)$ & $16(1)$ & $0(1)$ & $4(1)$ & $0(1)$ \\
\hline $\mathrm{C}(30)$ & $26(1)$ & $21(1)$ & $29(1)$ & $8(1)$ & $13(1)$ & $6(1)$ \\
\hline $\mathrm{C}(32)$ & $18(1)$ & $16(1)$ & $23(1)$ & $-3(1)$ & $7(1)$ & $2(1)$ \\
\hline $\mathrm{C}(31)$ & $23(1)$ & $20(1)$ & $19(1)$ & $2(1)$ & $11(1)$ & $5(1)$ \\
\hline $\mathrm{C}(33)$ & $22(1)$ & $18(1)$ & $23(1)$ & $-2(1)$ & $8(1)$ & $-4(1)$ \\
\hline $\mathrm{P}(3)$ & $14(1)$ & $12(1)$ & $15(1)$ & $-2(1)$ & $4(1)$ & $1(1)$ \\
\hline$C(34)$ & $24(1)$ & $22(1)$ & $17(1)$ & $-5(1)$ & $3(1)$ & $2(1)$ \\
\hline$C(24)$ & $16(1)$ & $27(1)$ & $30(1)$ & $6(1)$ & $7(1)$ & $7(1)$ \\
\hline $\mathrm{C}(5)$ & $22(1)$ & $18(1)$ & $20(1)$ & $-5(1)$ & $5(1)$ & $-7(1)$ \\
\hline
\end{tabular}




\subsection{Crystal Structure Determination of 1-Pd}

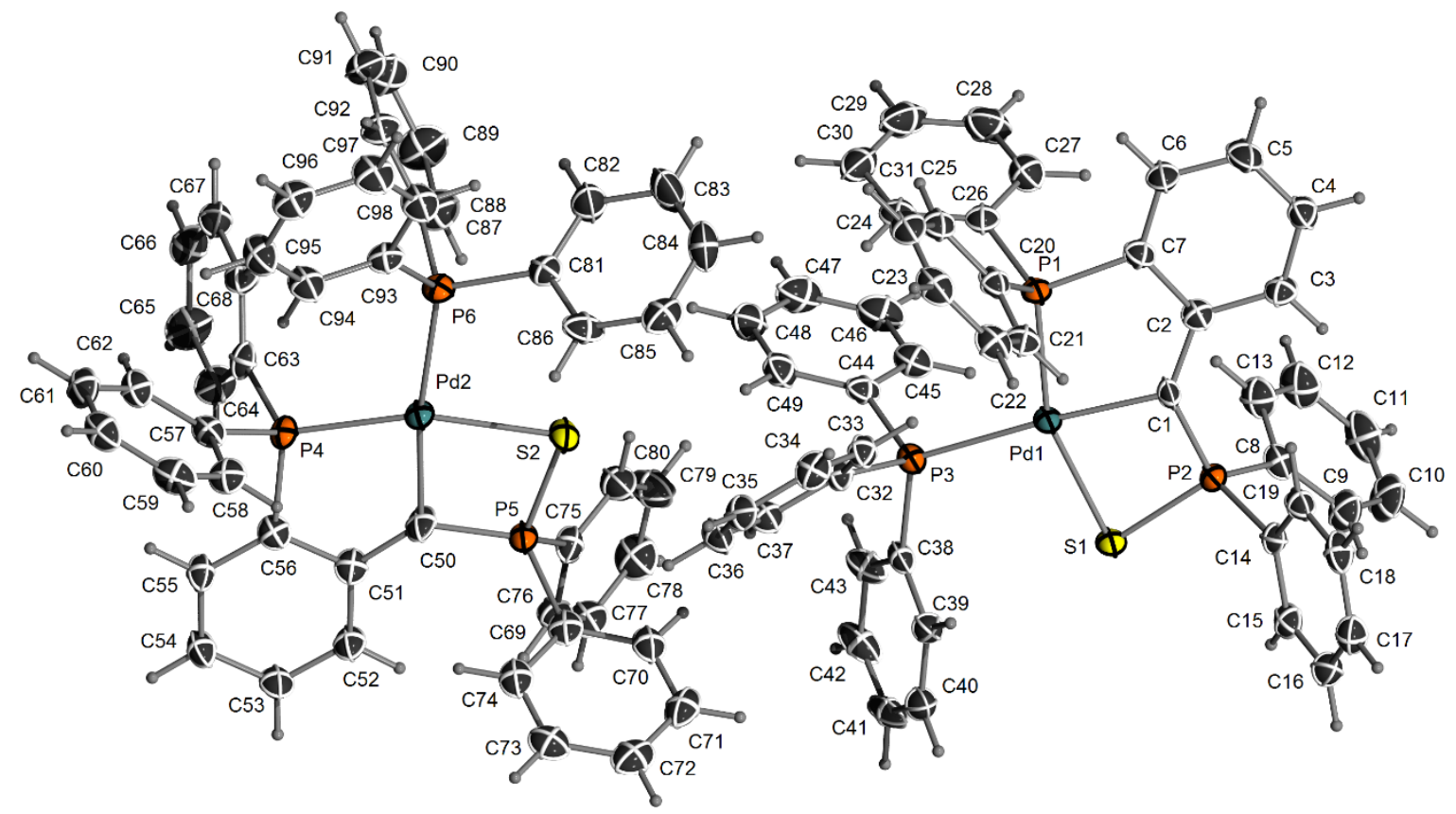

Figure S36. ORTEP Plot of compound 1-Pd. Ellipsoids are drawn at the $50 \%$ probability level.

Table S15. Atomic coordinates ( x 10 $)$ and equivalent isotropic displacement parameters $\left(\AA^{2} \times 10^{3}\right)$ for compound 1-Pd. $\mathrm{U}(\mathrm{eq})$ is defined as one third of the trace of the orthogonalized $\mathrm{U}_{\mathrm{ij}}$ tensor.

\begin{tabular}{ccccc}
\hline & $\mathrm{x}$ & $\mathrm{y}$ & $\mathrm{z}$ & $\mathrm{U}(\mathrm{eq})$ \\
\hline $\mathrm{Pd}(1)$ & $1981(1)$ & $2294(1)$ & $8285(1)$ & $18(1)$ \\
$\mathrm{Pd}(2)$ & $6373(1)$ & $2407(1)$ & $3284(1)$ & $20(1)$ \\
$\mathrm{S}(1)$ & $2704(1)$ & $1601(1)$ & $9606(1)$ & $23(1)$ \\
$\mathrm{S}(2)$ & $5625(1)$ & $2406(1)$ & $4606(1)$ & $30(1)$ \\
$\mathrm{P}(1)$ & $871(1)$ & $3097(1)$ & $7355(1)$ & $21(1)$ \\
$\mathrm{P}(2)$ & $1398(1)$ & $2011(1)$ & $10000(1)$ & $20(1)$ \\
$\mathrm{P}(3)$ & $3310(1)$ & $2089(1)$ & $7466(1)$ & $20(1)$ \\
$\mathrm{P}(4)$ & $7508(1)$ & $2340(1)$ & $2331(1)$ & $20(1)$ \\
$\mathrm{P}(5)$ & $6919(1)$ & $1887(1)$ & $4982(1)$ & $25(1)$ \\
$\mathrm{P}(6)$ & $4994(1)$ & $3059(1)$ & $2570(1)$ & $21(1)$ \\
$\mathrm{C}(1)$ & $897(2)$ & $2472(2)$ & $9079(2)$ & $23(1)$ \\
$\mathrm{C}(2)$ & $18(2)$ & $3040(2)$ & $8824(2)$ & $23(1)$ \\
$\mathrm{C}(3)$ & $-733(2)$ & $3281(2)$ & $9357(2)$ & $25(1)$ \\
$\mathrm{C}(4)$ & $-1575(2)$ & $3813(3)$ & $9040(2)$ & $29(1)$ \\
$\mathrm{C}(5)$ & $-1717(3)$ & $4126(3)$ & $8191(2)$ & $37(1)$ \\
$\mathrm{C}(6)$ & $-997(2)$ & $3920(3)$ & $7654(2)$ & $32(1)$ \\
$\mathrm{C}(7)$ & $-142(2)$ & $3409(2)$ & $7959(2)$ & $24(1)$ \\
$\mathrm{C}(8)$ & $1197(2)$ & $2794(2)$ & $10636(2)$ & $25(1)$ \\
$\mathrm{C}(9)$ & $1213(3)$ & $2535(3)$ & $11493(2)$ & $37(1)$ \\
$\mathrm{C}(10)$ & $1133(3)$ & $3173(3)$ & $11917(3)$ & $45(1)$ \\
$\mathrm{C}(11)$ & $1034(3)$ & $4062(3)$ & $11493(3)$ & $46(1)$ \\
$\mathrm{C}(12)$ & $974(3)$ & $4341(3)$ & $10640(3)$ & $44(1)$ \\
$\mathrm{C}(13)$ & $1049(3)$ & $3706(3)$ & $10218(3)$ & $36(1)$ \\
$\mathrm{C}(14)$ & $1166(2)$ & $1015(2)$ & $10681(2)$ & $22(1)$ \\
$\mathrm{C}(15)$ & $1764(2)$ & $397(2)$ & $11298(2)$ & $26(1)$ \\
$\mathrm{C}(16)$ & $1635(3)$ & $-410(3)$ & $11762(2)$ & $30(1)$ \\
$\mathrm{C}(17)$ & $903(3)$ & $-600(3)$ & $11607(2)$ & $31(1)$ \\
\hline & & & & \\
\hline
\end{tabular}




\begin{tabular}{|c|c|c|c|c|}
\hline $\mathrm{C}(18)$ & 297(2) & $24(3)$ & $11005(2)$ & $31(1)$ \\
\hline$C(19)$ & $430(2)$ & $825(2)$ & $10541(2)$ & $26(1)$ \\
\hline$C(20)$ & $664(2)$ & $2439(2)$ & $6743(2)$ & $24(1)$ \\
\hline$C(21)$ & $692(2)$ & $1555(2)$ & $7182(2)$ & $27(1)$ \\
\hline$C(22)$ & $581(2)$ & $969(3)$ & $6787(2)$ & $31(1)$ \\
\hline$C(23)$ & $433(2)$ & $1270(3)$ & $5940(2)$ & $32(1)$ \\
\hline$C(24)$ & $389(2)$ & $2146(3)$ & $5502(2)$ & $34(1)$ \\
\hline$C(25)$ & 493(2) & $2739(3)$ & $5900(2)$ & $28(1)$ \\
\hline$C(26)$ & $949(2)$ & $4158(2)$ & $6651(2)$ & $27(1)$ \\
\hline$C(27)$ & $535(3)$ & 4979(3) & $6877(3)$ & $37(1)$ \\
\hline$C(28)$ & $668(3)$ & $5774(3)$ & $6408(3)$ & $48(1)$ \\
\hline$C(29)$ & 1193(3) & $5785(3)$ & $5692(3)$ & $51(1)$ \\
\hline$C(30)$ & $1598(3)$ & $4974(3)$ & $5463(3)$ & $43(1)$ \\
\hline$C(31)$ & $1486(3)$ & $4167(3)$ & $5945(2)$ & $34(1)$ \\
\hline$C(32)$ & $3571(2)$ & $1327(2)$ & $6795(2)$ & 21(1) \\
\hline$C(33)$ & $2915(2)$ & $1006(2)$ & $6674(2)$ & $26(1)$ \\
\hline$C(34)$ & $3115(3)$ & $351(2)$ & $6234(2)$ & $28(1)$ \\
\hline$C(35)$ & $3965(3)$ & $15(2)$ & $5918(2)$ & $29(1)$ \\
\hline$C(36)$ & $4622(2)$ & $338(3)$ & $6029(2)$ & $31(1)$ \\
\hline$C(37)$ & $4424(2)$ & $992(2)$ & $6461(2)$ & $27(1)$ \\
\hline$C(38)$ & $4348(2)$ & $1567(2)$ & $8059(2)$ & $23(1)$ \\
\hline C(39) & $4535(2)$ & $675(2)$ & $8578(2)$ & $28(1)$ \\
\hline $\mathrm{C}(40)$ & $5316(3)$ & $252(3)$ & $9030(2)$ & $34(1)$ \\
\hline$C(41)$ & $5908(3)$ & $697(3)$ & $8979(2)$ & $36(1)$ \\
\hline$C(42)$ & $5740(2)$ & 1571(3) & $8450(3)$ & $38(1)$ \\
\hline$C(43)$ & 4957(3) & $2007(3)$ & 7991(3) & $32(1)$ \\
\hline$C(44)$ & $3404(2)$ & $3178(2)$ & $6838(2)$ & $24(1)$ \\
\hline$C(45)$ & $3099(2)$ & $3893(2)$ & $7214(2)$ & $31(1)$ \\
\hline$C(46)$ & $3209(3)$ & $4724(3)$ & $6820(3)$ & $40(1)$ \\
\hline$C(47)$ & $3614(3)$ & $4849(3)$ & $6047(3)$ & $45(1)$ \\
\hline$C(48)$ & 3904(3) & $4147(3)$ & $5660(3)$ & $40(1)$ \\
\hline$C(49)$ & $3806(2)$ & $3307(3)$ & $6055(2)$ & $33(1)$ \\
\hline$C(50)$ & $7452(2)$ & $1879(3)$ & $4051(2)$ & $29(1)$ \\
\hline$C(51)$ & $8342(2)$ & $1630(2)$ & $3779(2)$ & $25(1)$ \\
\hline$C(52)$ & $9106(2)$ & $1228(3)$ & $4287(2)$ & $30(1)$ \\
\hline$C(53)$ & $9959(2)$ & $950(3)$ & $3952(2)$ & $30(1)$ \\
\hline$C(54)$ & $10114(2)$ & $1057(3)$ & $3105(2)$ & $30(1)$ \\
\hline$C(55)$ & $9384(2)$ & $1472(2)$ & $2584(2)$ & $26(1)$ \\
\hline$C(56)$ & $8519(2)$ & $1759(2)$ & $2918(2)$ & $23(1)$ \\
\hline$C(57)$ & $7662(2)$ & $1738(2)$ & $1537(2)$ & $23(1)$ \\
\hline$C(58)$ & $7590(2)$ & $869(2)$ & 1791(2) & $29(1)$ \\
\hline C(59) & $7670(3)$ & $384(2)$ & $1219(3)$ & $32(1)$ \\
\hline$C(60)$ & $7824(2)$ & $765(3)$ & $390(2)$ & $32(1)$ \\
\hline$C(61)$ & $7909(3)$ & $1624(3)$ & $137(2)$ & $33(1)$ \\
\hline$C(62)$ & $7826(2)$ & $2104(2)$ & $719(2)$ & $27(1)$ \\
\hline$C(63)$ & $7535(2)$ & $3472(2)$ & $1751(2)$ & $25(1)$ \\
\hline$C(64)$ & $8217(3)$ & $3770(3)$ & $1842(2)$ & $35(1)$ \\
\hline$C(65)$ & $8189(3)$ & $4653(3)$ & $1422(3)$ & $43(1)$ \\
\hline$C(66)$ & $7477(3)$ & $5254(3)$ & $915(2)$ & $37(1)$ \\
\hline$C(67)$ & $6804(3)$ & $4959(3)$ & $816(2)$ & $33(1)$ \\
\hline$C(68)$ & $6829(2)$ & $4076(3)$ & $1231(2)$ & $29(1)$ \\
\hline C(69) & $7128(2)$ & $776(3)$ & $5731(2)$ & $27(1)$ \\
\hline$C(70)$ & $6719(2)$ & $702(3)$ & $6512(2)$ & $30(1)$ \\
\hline $\mathrm{C}(71)$ & $6790(3)$ & $-151(3)$ & $7045(2)$ & $36(1)$ \\
\hline$C(72)$ & $7306(3)$ & $-942(3)$ & $6819(3)$ & $38(1)$ \\
\hline$C(73)$ & $7747(3)$ & $-872(3)$ & $6056(2)$ & $35(1)$ \\
\hline
\end{tabular}




\begin{tabular}{lllll}
\hline $\mathrm{C}(74)$ & $7654(2)$ & $-20(3)$ & $5514(2)$ & $30(1)$ \\
$\mathrm{C}(75)$ & $7082(2)$ & $2550(2)$ & $5614(2)$ & $26(1)$ \\
$\mathrm{C}(76)$ & $7904(2)$ & $2260(3)$ & $6001(2)$ & $30(1)$ \\
$\mathrm{C}(77)$ & $8040(3)$ & $2770(3)$ & $6467(2)$ & $34(1)$ \\
$\mathrm{C}(78)$ & $7364(3)$ & $3542(3)$ & $6567(3)$ & $44(1)$ \\
$\mathrm{C}(79)$ & $6541(3)$ & $3827(3)$ & $6202(3)$ & $49(1)$ \\
$\mathrm{C}(80)$ & $6407(3)$ & $3321(3)$ & $5728(2)$ & $38(1)$ \\
$\mathrm{C}(81)$ & $4025(2)$ & $3073(2)$ & $3274(2)$ & $24(1)$ \\
$\mathrm{C}(82)$ & $3313(3)$ & $3854(3)$ & $3290(3)$ & $45(1)$ \\
$\mathrm{C}(83)$ & $2570(4)$ & $3804(3)$ & $3798(4)$ & $62(2)$ \\
$\mathrm{C}(84)$ & $2552(3)$ & $2980(3)$ & $4299(3)$ & $43(1)$ \\
$\mathrm{C}(85)$ & $3274(3)$ & $2196(3)$ & $4311(2)$ & $35(1)$ \\
$\mathrm{C}(86)$ & $4007(2)$ & $2243(3)$ & $3799(2)$ & $32(1)$ \\
$\mathrm{C}(87)$ & $4750(2)$ & $4277(2)$ & $2055(2)$ & $25(1)$ \\
$\mathrm{C}(88)$ & $5016(3)$ & $4786(3)$ & $2442(3)$ & $38(1)$ \\
$\mathrm{C}(89)$ & $4854(4)$ & $5700(3)$ & $2085(3)$ & $47(1)$ \\
$\mathrm{C}(90)$ & $4439(3)$ & $6140(3)$ & $1319(3)$ & $44(1)$ \\
$\mathrm{C}(91)$ & $4180(3)$ & $5642(3)$ & $931(3)$ & $39(1)$ \\
$\mathrm{C}(92)$ & $4332(3)$ & $4718(3)$ & $1289(2)$ & $32(1)$ \\
$\mathrm{C}(93)$ & $4730(2)$ & $2652(2)$ & $1767(2)$ & $24(1)$ \\
$\mathrm{C}(94)$ & $5412(2)$ & $2301(3)$ & $1246(2)$ & $32(1)$ \\
$\mathrm{C}(95)$ & $5232(3)$ & $2080(3)$ & $566(2)$ & $35(1)$ \\
$\mathrm{C}(96)$ & $4364(3)$ & $2209(3)$ & $407(2)$ & $34(1)$ \\
$\mathrm{C}(97)$ & $3680(3)$ & $2547(3)$ & $932(3)$ & $36(1)$ \\
$\mathrm{C}(98)$ & $3855(3)$ & $2768(3)$ & $1609(2)$ & $33(1)$ \\
\hline
\end{tabular}

Table S16. Anisotropic displacement parameters $\left(\AA^{2} \times 10^{3}\right)$ for compound 1-Pd. The anisotropic displacement factor exponent takes the form: $-2 \pi^{2}\left[\mathrm{~h}^{2} \mathrm{a}^{* 2} \mathrm{U}^{11}+\ldots+2 \mathrm{~h} \mathrm{k} \mathrm{a}^{*} \mathrm{~b}^{*} \mathrm{U}^{12}\right]$.

\begin{tabular}{ccccccc}
\hline & $\mathrm{U}^{11}$ & $\mathrm{U}^{22}$ & $\mathrm{U}^{33}$ & $\mathrm{U}^{23}$ & $\mathrm{U}^{13}$ & $\mathrm{U}^{12}$ \\
\hline $\mathrm{Pd}(1)$ & $16(1)$ & $23(1)$ & $18(1)$ & $-9(1)$ & $-2(1)$ & $-6(1)$ \\
$\mathrm{Pd}(2)$ & $19(1)$ & $27(1)$ & $18(1)$ & $-8(1)$ & $-2(1)$ & $-9(1)$ \\
$\mathrm{S}(1)$ & $18(1)$ & $31(1)$ & $21(1)$ & $-9(1)$ & $-3(1)$ & $-7(1)$ \\
$\mathrm{S}(2)$ & $18(1)$ & $51(1)$ & $23(1)$ & $-13(1)$ & $-1(1)$ & $-10(1)$ \\
$\mathrm{P}(1)$ & $18(1)$ & $27(1)$ & $20(1)$ & $-9(1)$ & $-3(1)$ & $-6(1)$ \\
$\mathrm{P}(2)$ & $18(1)$ & $24(1)$ & $19(1)$ & $-9(1)$ & $-1(1)$ & $-6(1)$ \\
$\mathrm{P}(3)$ & $17(1)$ & $23(1)$ & $22(1)$ & $-10(1)$ & $0(1)$ & $-7(1)$ \\
$\mathrm{P}(4)$ & $23(1)$ & $25(1)$ & $18(1)$ & $-9(1)$ & $0(1)$ & $-11(1)$ \\
$\mathrm{P}(5)$ & $18(1)$ & $39(1)$ & $19(1)$ & $-10(1)$ & $-1(1)$ & $-9(1)$ \\
$\mathrm{P}(6)$ & $21(1)$ & $23(1)$ & $22(1)$ & $-9(1)$ & $-5(1)$ & $-7(1)$ \\
$\mathrm{C}(1)$ & $18(2)$ & $27(2)$ & $22(2)$ & $-12(1)$ & $6(1)$ & $-5(1)$ \\
$\mathrm{C}(2)$ & $22(2)$ & $26(2)$ & $24(2)$ & $-10(1)$ & $-2(1)$ & $-9(1)$ \\
$\mathrm{C}(3)$ & $24(2)$ & $29(2)$ & $23(2)$ & $-7(1)$ & $-3(1)$ & $-10(1)$ \\
$\mathrm{C}(4)$ & $22(2)$ & $37(2)$ & $29(2)$ & $-12(2)$ & $2(1)$ & $-9(2)$ \\
$\mathrm{C}(5)$ & $20(2)$ & $49(2)$ & $35(2)$ & $-14(2)$ & $-6(2)$ & $-3(2)$ \\
$\mathrm{C}(6)$ & $23(2)$ & $45(2)$ & $23(2)$ & $-9(2)$ & $-5(1)$ & $-6(2)$ \\
$\mathrm{C}(7)$ & $19(2)$ & $28(2)$ & $25(2)$ & $-8(1)$ & $-2(1)$ & $-7(1)$ \\
$\mathrm{C}(8)$ & $22(2)$ & $28(2)$ & $29(2)$ & $-14(1)$ & $3(1)$ & $-9(1)$ \\
$\mathrm{C}(9)$ & $48(2)$ & $35(2)$ & $29(2)$ & $-16(2)$ & $3(2)$ & $-13(2)$ \\
$\mathrm{C}(10)$ & $63(3)$ & $48(2)$ & $29(2)$ & $-22(2)$ & $4(2)$ & $-17(2)$ \\
$\mathrm{C}(11)$ & $49(3)$ & $45(2)$ & $57(3)$ & $-36(2)$ & $12(2)$ & $-19(2)$ \\
$\mathrm{C}(12)$ & $53(3)$ & $31(2)$ & $51(3)$ & $-19(2)$ & $9(2)$ & $-16(2)$ \\
$\mathrm{C}(13)$ & $40(2)$ & $34(2)$ & $38(2)$ & $-16(2)$ & $2(2)$ & $-13(2)$ \\
$\mathrm{C}(14)$ & $23(2)$ & $24(2)$ & $18(2)$ & $-10(1)$ & $0(1)$ & $-5(1)$ \\
$\mathrm{C}(15)$ & $26(2)$ & $31(2)$ & $23(2)$ & $-13(1)$ & $0(1)$ & $-9(1)$ \\
$\mathrm{C}(16)$ & $32(2)$ & $31(2)$ & $25(2)$ & $-8(2)$ & $-1(1)$ & $-6(2)$ \\
\hline & & & & & &
\end{tabular}




\begin{tabular}{|c|c|c|c|c|c|c|}
\hline $\mathrm{C}(17)$ & $35(2)$ & $30(2)$ & $30(2)$ & $-10(2)$ & $7(2)$ & $-14(2)$ \\
\hline $\mathrm{C}(18)$ & $27(2)$ & $41(2)$ & $33(2)$ & $-16(2)$ & $7(1)$ & $-17(2)$ \\
\hline $\mathrm{C}(19)$ & $22(2)$ & $31(2)$ & $25(2)$ & $-13(1)$ & 1(1) & $-6(1)$ \\
\hline $\mathrm{C}(20)$ & $18(2)$ & $31(2)$ & $25(2)$ & $-12(1)$ & $-2(1)$ & $-5(1)$ \\
\hline $\mathrm{C}(21)$ & $21(2)$ & $34(2)$ & $28(2)$ & $-8(2)$ & $-4(1)$ & $-10(1)$ \\
\hline $\mathrm{C}(22)$ & $25(2)$ & $33(2)$ & $38(2)$ & $-15(2)$ & $-1(2)$ & $-12(2)$ \\
\hline $\mathrm{C}(23)$ & $24(2)$ & $43(2)$ & $39(2)$ & $-25(2)$ & $-4(2)$ & $-9(2)$ \\
\hline $\mathrm{C}(24)$ & $28(2)$ & $48(2)$ & $27(2)$ & $-17(2)$ & $-6(1)$ & $-8(2)$ \\
\hline $\mathrm{C}(25)$ & $22(2)$ & $35(2)$ & $28(2)$ & $-10(2)$ & $-3(1)$ & $-7(2)$ \\
\hline$C(26)$ & $22(2)$ & $33(2)$ & $24(2)$ & $-7(1)$ & $-5(1)$ & $-8(1)$ \\
\hline$C(27)$ & $31(2)$ & $37(2)$ & $38(2)$ & $-10(2)$ & $2(2)$ & $-9(2)$ \\
\hline$C(28)$ & $44(2)$ & $29(2)$ & $62(3)$ & $-6(2)$ & $-4(2)$ & $-6(2)$ \\
\hline$C(29)$ & $45(3)$ & $43(2)$ & $52(3)$ & $13(2)$ & $-8(2)$ & $-17(2)$ \\
\hline$C(30)$ & $37(2)$ & $54(3)$ & $31(2)$ & $5(2)$ & $-5(2)$ & $-19(2)$ \\
\hline$C(31)$ & $25(2)$ & $44(2)$ & $30(2)$ & $-6(2)$ & $-6(1)$ & $-9(2)$ \\
\hline$C(32)$ & $23(2)$ & $21(2)$ & $19(2)$ & $-7(1)$ & $-1(1)$ & $-7(1)$ \\
\hline$C(33)$ & $21(2)$ & $34(2)$ & $23(2)$ & $-9(1)$ & $1(1)$ & $-11(1)$ \\
\hline$C(34)$ & $34(2)$ & $32(2)$ & $26(2)$ & $-10(1)$ & $-1(1)$ & $-18(2)$ \\
\hline$C(35)$ & $36(2)$ & $28(2)$ & $25(2)$ & $-13(1)$ & $-2(1)$ & $-7(2)$ \\
\hline$C(36)$ & $24(2)$ & $37(2)$ & $31(2)$ & $-18(2)$ & $3(1)$ & $-6(2)$ \\
\hline$C(37)$ & $25(2)$ & $31(2)$ & $29(2)$ & $-11(2)$ & $-2(1)$ & $-9(2)$ \\
\hline$C(38)$ & $15(2)$ & $32(2)$ & $24(2)$ & $-16(1)$ & $2(1)$ & $-6(1)$ \\
\hline C(39) & $23(2)$ & $31(2)$ & $26(2)$ & $-8(1)$ & $-1(1)$ & $-6(1)$ \\
\hline$C(40)$ & $30(2)$ & $37(2)$ & $26(2)$ & $-10(2)$ & $-1(2)$ & $-2(2)$ \\
\hline$C(41)$ & $27(2)$ & $41(2)$ & $40(2)$ & $-26(2)$ & $-9(2)$ & $4(2)$ \\
\hline$C(42)$ & $21(2)$ & $48(2)$ & $57(3)$ & $-34(2)$ & $-5(2)$ & $-8(2)$ \\
\hline$C(43)$ & $27(2)$ & $28(2)$ & $45(2)$ & $-19(2)$ & $-6(2)$ & $-5(2)$ \\
\hline$C(44)$ & $17(2)$ & $23(2)$ & $30(2)$ & $-4(1)$ & $-3(1)$ & $-6(1)$ \\
\hline$C(45)$ & $27(2)$ & $29(2)$ & $37(2)$ & $-11(2)$ & $-3(2)$ & $-8(2)$ \\
\hline$C(46)$ & $39(2)$ & $27(2)$ & $57(3)$ & $-12(2)$ & $-6(2)$ & $-11(2)$ \\
\hline$C(47)$ & $38(2)$ & $30(2)$ & $61(3)$ & $0(2)$ & $-2(2)$ & $-16(2)$ \\
\hline$C(48)$ & $29(2)$ & $34(2)$ & $47(2)$ & $-1(2)$ & $6(2)$ & $-11(2)$ \\
\hline C(49) & $24(2)$ & $34(2)$ & $39(2)$ & $-10(2)$ & $5(2)$ & $-10(2)$ \\
\hline$C(50)$ & $25(2)$ & $49(2)$ & $18(2)$ & $-11(2)$ & $-2(1)$ & $-15(2)$ \\
\hline$C(51)$ & $25(2)$ & $31(2)$ & $26(2)$ & $-10(1)$ & $-1(1)$ & $-14(2)$ \\
\hline$C(52)$ & $25(2)$ & $43(2)$ & $24(2)$ & $-12(2)$ & $-1(1)$ & $-12(2)$ \\
\hline$C(53)$ & $22(2)$ & $42(2)$ & $28(2)$ & $-12(2)$ & $-3(1)$ & $-10(2)$ \\
\hline$C(54)$ & $21(2)$ & $40(2)$ & $32(2)$ & $-16(2)$ & $4(1)$ & $-10(2)$ \\
\hline$C(55)$ & $25(2)$ & $37(2)$ & $20(2)$ & $-12(1)$ & $3(1)$ & $-14(2)$ \\
\hline$C(56)$ & $24(2)$ & $24(2)$ & $26(2)$ & $-11(1)$ & $-1(1)$ & $-11(1)$ \\
\hline$C(57)$ & $19(2)$ & $27(2)$ & $27(2)$ & $-13(1)$ & $-3(1)$ & $-7(1)$ \\
\hline$C(58)$ & $32(2)$ & $30(2)$ & $29(2)$ & $-11(2)$ & $-5(1)$ & $-12(2)$ \\
\hline C(59) & $33(2)$ & $26(2)$ & $45(2)$ & $-19(2)$ & $-7(2)$ & $-9(2)$ \\
\hline$C(60)$ & $28(2)$ & $39(2)$ & $37(2)$ & $-25(2)$ & $-3(2)$ & $-8(2)$ \\
\hline$C(61)$ & $33(2)$ & $44(2)$ & $27(2)$ & $-16(2)$ & $1(2)$ & $-14(2)$ \\
\hline$C(62)$ & $29(2)$ & $29(2)$ & $29(2)$ & $-13(2)$ & 1(1) & $-12(2)$ \\
\hline$C(63)$ & $33(2)$ & $29(2)$ & $19(2)$ & $-12(1)$ & $3(1)$ & $-14(2)$ \\
\hline$C(64)$ & $40(2)$ & $34(2)$ & $37(2)$ & $-7(2)$ & $-6(2)$ & $-18(2)$ \\
\hline$C(65)$ & $49(2)$ & $41(2)$ & $49(2)$ & $-7(2)$ & $-8(2)$ & $-28(2)$ \\
\hline$C(66)$ & $52(2)$ & $27(2)$ & $34(2)$ & $-9(2)$ & $3(2)$ & $-18(2)$ \\
\hline$C(67)$ & $36(2)$ & $32(2)$ & $28(2)$ & $-8(2)$ & $2(2)$ & $-10(2)$ \\
\hline$C(68)$ & $29(2)$ & $34(2)$ & $26(2)$ & $-9(2)$ & $-2(1)$ & $-12(2)$ \\
\hline$C(69)$ & $21(2)$ & $44(2)$ & $23(2)$ & $-13(2)$ & $-6(1)$ & $-14(2)$ \\
\hline$C(70)$ & $22(2)$ & $44(2)$ & $28(2)$ & $-15(2)$ & $-1(1)$ & $-12(2)$ \\
\hline$C(71)$ & $33(2)$ & $55(2)$ & $25(2)$ & $-9(2)$ & $-4(2)$ & $-23(2)$ \\
\hline$C(72)$ & $34(2)$ & $46(2)$ & $36(2)$ & $-5(2)$ & $-11(2)$ & $-21(2)$ \\
\hline
\end{tabular}




\begin{tabular}{lllllll}
\hline $\mathrm{C}(73)$ & $28(2)$ & $38(2)$ & $42(2)$ & $-14(2)$ & $-7(2)$ & $-12(2)$ \\
$\mathrm{C}(74)$ & $24(2)$ & $47(2)$ & $27(2)$ & $-17(2)$ & $-2(1)$ & $-16(2)$ \\
$\mathrm{C}(75)$ & $26(2)$ & $37(2)$ & $21(2)$ & $-9(1)$ & $2(1)$ & $-16(2)$ \\
$\mathrm{C}(76)$ & $26(2)$ & $35(2)$ & $31(2)$ & $-13(2)$ & $-3(1)$ & $-9(2)$ \\
$\mathrm{C}(77)$ & $32(2)$ & $42(2)$ & $34(2)$ & $-15(2)$ & $-7(2)$ & $-14(2)$ \\
$\mathrm{C}(78)$ & $48(3)$ & $50(2)$ & $43(2)$ & $-24(2)$ & $-5(2)$ & $-19(2)$ \\
$\mathrm{C}(79)$ & $46(3)$ & $47(2)$ & $50(3)$ & $-26(2)$ & $-9(2)$ & $1(2)$ \\
$\mathrm{C}(80)$ & $33(2)$ & $46(2)$ & $35(2)$ & $-18(2)$ & $-5(2)$ & $-5(2)$ \\
$\mathrm{C}(81)$ & $24(2)$ & $27(2)$ & $24(2)$ & $-10(1)$ & $-4(1)$ & $-8(1)$ \\
$\mathrm{C}(82)$ & $50(3)$ & $29(2)$ & $42(2)$ & $-4(2)$ & $15(2)$ & $-6(2)$ \\
$\mathrm{C}(83)$ & $51(3)$ & $39(3)$ & $71(3)$ & $-12(2)$ & $30(3)$ & $2(2)$ \\
$\mathrm{C}(84)$ & $42(2)$ & $56(3)$ & $38(2)$ & $-23(2)$ & $16(2)$ & $-21(2)$ \\
$\mathrm{C}(85)$ & $36(2)$ & $43(2)$ & $32(2)$ & $-4(2)$ & $-7(2)$ & $-21(2)$ \\
$\mathrm{C}(86)$ & $22(2)$ & $31(2)$ & $42(2)$ & $-5(2)$ & $-7(2)$ & $-9(2)$ \\
$\mathrm{C}(87)$ & $28(2)$ & $29(2)$ & $23(2)$ & $-10(1)$ & $2(1)$ & $-14(2)$ \\
$\mathrm{C}(88)$ & $52(2)$ & $33(2)$ & $36(2)$ & $-14(2)$ & $-8(2)$ & $-16(2)$ \\
$\mathrm{C}(89)$ & $67(3)$ & $34(2)$ & $48(3)$ & $-12(2)$ & $-8(2)$ & $-24(2)$ \\
$\mathrm{C}(90)$ & $60(3)$ & $25(2)$ & $44(2)$ & $-6(2)$ & $2(2)$ & $-15(2)$ \\
$\mathrm{C}(91)$ & $48(2)$ & $33(2)$ & $32(2)$ & $-1(2)$ & $-8(2)$ & $-11(2)$ \\
$\mathrm{C}(92)$ & $34(2)$ & $33(2)$ & $31(2)$ & $-7(2)$ & $-7(2)$ & $-12(2)$ \\
$\mathrm{C}(93)$ & $27(2)$ & $24(2)$ & $25(2)$ & $-7(1)$ & $-7(1)$ & $-10(1)$ \\
$\mathrm{C}(94)$ & $24(2)$ & $44(2)$ & $33(2)$ & $-19(2)$ & $-5(1)$ & $-11(2)$ \\
$\mathrm{C}(95)$ & $32(2)$ & $44(2)$ & $35(2)$ & $-23(2)$ & $-1(2)$ & $-11(2)$ \\
$\mathrm{C}(96)$ & $41(2)$ & $40(2)$ & $32(2)$ & $-15(2)$ & $-10(2)$ & $-19(2)$ \\
$\mathrm{C}(97)$ & $29(2)$ & $49(2)$ & $41(2)$ & $-19(2)$ & $-9(2)$ & $-16(2)$ \\
$\mathrm{C}(98)$ & $27(2)$ & $45(2)$ & $34(2)$ & $-18(2)$ & $-3(2)$ & $-13(2)$ \\
\hline & & & & & & \\
\hline
\end{tabular}

\subsection{Crystal Structure Determination of $\mathbf{3}_{\mathrm{Ni}}$}

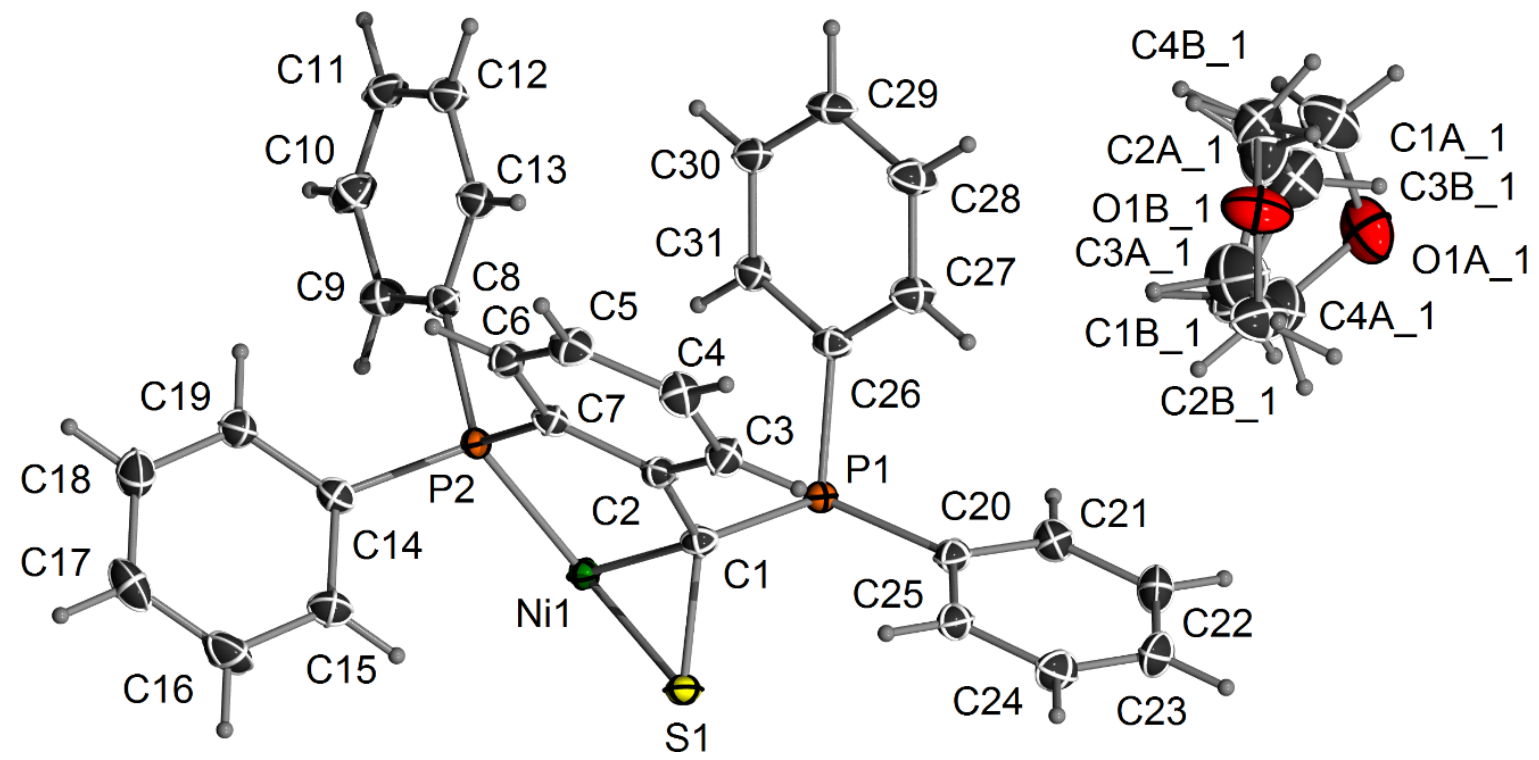

Figure S37. ORTEP Plot of compound $\mathbf{3}_{\mathrm{Ni}}$. Ellipsoids are drawn at the 50\% probability level. 
Table S17. Atomic coordinates ( $\left.\times 10^{4}\right)$ and equivalent isotropic displacement parameters $\left(\AA^{2} \times 10^{3}\right)$ for compound $\mathbf{3}_{\mathrm{Ni}}$. $\mathrm{U}(\mathrm{eq})$ is defined as one third of the trace of the orthogonalized $\mathrm{U}_{\mathrm{ij}}$ tensor.

\begin{tabular}{|c|c|c|c|c|}
\hline & $\mathrm{X}$ & $\mathrm{y}$ & $\mathrm{Z}$ & $\mathrm{U}(\mathrm{eq})$ \\
\hline $\mathrm{Ni}(1)$ & $4758(1)$ & $3906(1)$ & $4804(1)$ & $14(1)$ \\
\hline $\mathrm{S}(1)$ & $6429(1)$ & 3996(1) & $5964(1)$ & $17(1)$ \\
\hline$P(1)$ & $4504(1)$ & $5282(1)$ & $6642(1)$ & $13(1)$ \\
\hline$P(2)$ & $2859(1)$ & $2408(1)$ & $4676(1)$ & $14(1)$ \\
\hline $\mathrm{C}(1)$ & $4676(2)$ & $3828(1)$ & $6305(1)$ & $14(1)$ \\
\hline $\mathrm{C}(2)$ & $3714(2)$ & 2722(1) & $6704(1)$ & $16(1)$ \\
\hline $\mathrm{C}(3)$ & $3694(2)$ & $2448(2)$ & $7737(1)$ & $20(1)$ \\
\hline $\mathrm{C}(4)$ & $2804(2)$ & 1391(2) & $8059(1)$ & $22(1)$ \\
\hline$C(5)$ & $1914(2)$ & $592(1)$ & $7347(1)$ & $21(1)$ \\
\hline $\mathrm{C}(6)$ & $1900(2)$ & $864(1)$ & $6320(1)$ & $19(1)$ \\
\hline$C(7)$ & $2775(2)$ & $1929(1)$ & $5998(1)$ & $16(1)$ \\
\hline $\mathrm{C}(8)$ & $1060(2)$ & 2398(1) & $4276(1)$ & $17(1)$ \\
\hline $\mathrm{C}(9)$ & $850(2)$ & $2711(2)$ & $3269(1)$ & $21(1)$ \\
\hline$C(10)$ & $-495(2)$ & $2703(2)$ & $2908(1)$ & $24(1)$ \\
\hline $\mathrm{C}(11)$ & $-1635(2)$ & $2409(2)$ & $3550(2)$ & $24(1)$ \\
\hline$C(12)$ & $-1429(2)$ & $2123(2)$ & $4556(2)$ & $23(1)$ \\
\hline$C(13)$ & $-85(2)$ & 2113(1) & 4919(1) & $20(1)$ \\
\hline$C(14)$ & $3080(2)$ & $1132(1)$ & 3964(1) & $16(1)$ \\
\hline$C(15)$ & $4428(2)$ & $961(2)$ & $4026(1)$ & $24(1)$ \\
\hline$C(16)$ & $4663(2)$ & $-8(2)$ & $3535(2)$ & $30(1)$ \\
\hline$C(17)$ & $3562(2)$ & $-818(2)$ & $2977(2)$ & $30(1)$ \\
\hline $\mathrm{C}(18)$ & $2232(2)$ & $-650(2)$ & $2911(2)$ & $29(1)$ \\
\hline C(19) & $1988(2)$ & $322(2)$ & $3397(1)$ & $21(1)$ \\
\hline$C(20)$ & $5582(2)$ & $5854(1)$ & $7820(1)$ & $17(1)$ \\
\hline $\mathrm{C}(21)$ & $5700(2)$ & $7005(2)$ & $8148(1)$ & $22(1)$ \\
\hline$C(22)$ & $6579(2)$ & $7521(2)$ & $8997(2)$ & $28(1)$ \\
\hline $\mathrm{C}(23)$ & $7351(2)$ & $6891(2)$ & $9520(1)$ & $29(1)$ \\
\hline$C(24)$ & $7251(2)$ & $5756(2)$ & 9191(1) & $25(1)$ \\
\hline$C(25)$ & $6372(2)$ & $5236(2)$ & $8345(1)$ & $19(1)$ \\
\hline$C(26)$ & $2673(2)$ & 4984(1) & $7035(1)$ & $16(1)$ \\
\hline$C(27)$ & $2306(2)$ & $4956(2)$ & $8060(1)$ & $21(1)$ \\
\hline $\mathrm{C}(28)$ & $880(2)$ & $4659(2)$ & $8302(1)$ & $26(1)$ \\
\hline C(29) & $-182(2)$ & $4378(2)$ & $7529(2)$ & $24(1)$ \\
\hline$C(30)$ & $178(2)$ & $4406(2)$ & $6510(1)$ & $21(1)$ \\
\hline $\mathrm{C}(31)$ & $1600(2)$ & $4714(1)$ & $6260(1)$ & $18(1)$ \\
\hline O1A1 & 2861(3) & $9628(2)$ & $9937(2)$ & $45(1)$ \\
\hline C1A1 & $1454(5)$ & $8902(4)$ & $10061(4)$ & $46(1)$ \\
\hline C2A1 & $1566(9)$ & $7689(7)$ & $10238(12)$ & $50(2)$ \\
\hline C3A1 & $3030(6)$ & $7734(4)$ & $9848(5)$ & $56(1)$ \\
\hline C4A1 & $3531(7)$ & $8910(5)$ & $9392(5)$ & $50(1)$ \\
\hline O1B1 & $2268(4)$ & $7084(4)$ & $10633(3)$ & $42(1)$ \\
\hline C1B1 & $3594(6)$ & $7643(6)$ & $10195(4)$ & $37(1)$ \\
\hline $\mathrm{C} 2 \mathrm{~B} 1$ & $3288(7)$ & $8365(7)$ & $9342(5)$ & $36(1)$ \\
\hline $\mathrm{C} 3 \mathrm{~B} 1$ & $2032(7)$ & $8719(5)$ & $9772(5)$ & $41(1)$ \\
\hline C4B1 & $1229(9)$ & $7584(10)$ & $10240(14)$ & $31(2)$ \\
\hline
\end{tabular}


Table S18. Anisotropic displacement parameters $\left(\AA^{2} \times 10^{3}\right)$ for compound $\mathbf{3}_{\mathrm{Ni}}$. The anisotropic displacement factor exponent takes the form: $-2 \pi^{2}\left[\mathrm{~h}^{2} \mathrm{a}^{* 2} \mathrm{U}^{11}+\ldots+2 \mathrm{~h} \mathrm{k} \mathrm{a}^{*} \mathrm{~b}^{*} \mathrm{U}^{12}\right]$.

\begin{tabular}{|c|c|c|c|c|c|c|}
\hline & $\overline{U^{11}}$ & $\mathrm{U}^{22}$ & $\mathrm{U}^{33}$ & $\mathrm{U}^{23}$ & $\mathrm{U}^{13}$ & $\mathrm{U}^{12}$ \\
\hline $\mathrm{Ni}(1)$ & $12(1)$ & $14(1)$ & $14(1)$ & $1(1)$ & 1(1) & $2(1)$ \\
\hline $\mathrm{S}(1)$ & 11(1) & $21(1)$ & 19(1) & 1(1) & 1(1) & $6(1)$ \\
\hline $\mathrm{P}(1)$ & $11(1)$ & $14(1)$ & $14(1)$ & $0(1)$ & 1(1) & $4(1)$ \\
\hline $\mathrm{P}(2)$ & $11(1)$ & $14(1)$ & $16(1)$ & 1(1) & $1(1)$ & $3(1)$ \\
\hline $\mathrm{C}(1)$ & $13(1)$ & $14(1)$ & $16(1)$ & 2(1) & 1(1) & $4(1)$ \\
\hline $\mathrm{C}(2)$ & $13(1)$ & $15(1)$ & $20(1)$ & 1(1) & $3(1)$ & $6(1)$ \\
\hline $\mathrm{C}(3)$ & $20(1)$ & $20(1)$ & $19(1)$ & $0(1)$ & $0(1)$ & $4(1)$ \\
\hline $\mathrm{C}(4)$ & $26(1)$ & 21(1) & $19(1)$ & $4(1)$ & $3(1)$ & $6(1)$ \\
\hline $\mathrm{C}(5)$ & $20(1)$ & $16(1)$ & $27(1)$ & $5(1)$ & $6(1)$ & $3(1)$ \\
\hline $\mathrm{C}(6)$ & $16(1)$ & $16(1)$ & $23(1)$ & $0(1)$ & $2(1)$ & $4(1)$ \\
\hline $\mathrm{C}(7)$ & $14(1)$ & $17(1)$ & $18(1)$ & 1(1) & 1(1) & $7(1)$ \\
\hline $\mathrm{C}(8)$ & $14(1)$ & $13(1)$ & $23(1)$ & $-2(1)$ & $0(1)$ & $5(1)$ \\
\hline $\mathrm{C}(9)$ & $17(1)$ & $21(1)$ & $25(1)$ & $3(1)$ & $1(1)$ & $5(1)$ \\
\hline$C(10)$ & $21(1)$ & $21(1)$ & $29(1)$ & $3(1)$ & $-6(1)$ & $7(1)$ \\
\hline $\mathrm{C}(11)$ & $15(1)$ & $20(1)$ & $38(1)$ & $-2(1)$ & $-5(1)$ & $7(1)$ \\
\hline $\mathrm{C}(12)$ & $16(1)$ & $21(1)$ & $32(1)$ & $-2(1)$ & $2(1)$ & $6(1)$ \\
\hline C(13) & $17(1)$ & $20(1)$ & $24(1)$ & $-2(1)$ & $2(1)$ & $6(1)$ \\
\hline C(14) & $17(1)$ & $18(1)$ & $16(1)$ & $2(1)$ & $4(1)$ & $6(1)$ \\
\hline$C(15)$ & $19(1)$ & $26(1)$ & $28(1)$ & $-1(1)$ & $-1(1)$ & $10(1)$ \\
\hline C(16) & $29(1)$ & $35(1)$ & $35(1)$ & $0(1)$ & $4(1)$ & 21(1) \\
\hline$C(17)$ & $41(1)$ & $26(1)$ & $29(1)$ & $-2(1)$ & $8(1)$ & $17(1)$ \\
\hline C(18) & $32(1)$ & $23(1)$ & $29(1)$ & $-7(1)$ & $2(1)$ & $6(1)$ \\
\hline C(19) & $18(1)$ & $20(1)$ & $24(1)$ & $-2(1)$ & $2(1)$ & $5(1)$ \\
\hline $\mathrm{C}(20)$ & $14(1)$ & $21(1)$ & $16(1)$ & $0(1)$ & $2(1)$ & 2(1) \\
\hline $\mathrm{C}(21)$ & $22(1)$ & $23(1)$ & $22(1)$ & $-2(1)$ & $1(1)$ & $7(1)$ \\
\hline $\mathrm{C}(22)$ & $29(1)$ & $27(1)$ & $28(1)$ & $-10(1)$ & $-3(1)$ & $7(1)$ \\
\hline $\mathrm{C}(23)$ & $24(1)$ & $40(1)$ & $20(1)$ & $-10(1)$ & $-5(1)$ & $7(1)$ \\
\hline $\mathrm{C}(24)$ & $22(1)$ & $36(1)$ & $19(1)$ & $1(1)$ & $-2(1)$ & $10(1)$ \\
\hline $\mathrm{C}(25)$ & $16(1)$ & $22(1)$ & $17(1)$ & 1(1) & 1(1) & $4(1)$ \\
\hline $\mathrm{C}(26)$ & $14(1)$ & $14(1)$ & $20(1)$ & 2(1) & 2(1) & $6(1)$ \\
\hline $\mathrm{C}(27)$ & $17(1)$ & $28(1)$ & $20(1)$ & $4(1)$ & 1(1) & $7(1)$ \\
\hline $\mathrm{C}(28)$ & $20(1)$ & $37(1)$ & $21(1)$ & $5(1)$ & $6(1)$ & $10(1)$ \\
\hline C(29) & $14(1)$ & $26(1)$ & $32(1)$ & $4(1)$ & $6(1)$ & $6(1)$ \\
\hline $\mathrm{C}(30)$ & $14(1)$ & $21(1)$ & $26(1)$ & $-2(1)$ & $0(1)$ & $6(1)$ \\
\hline $\mathrm{C}(31)$ & $16(1)$ & $18(1)$ & $20(1)$ & $0(1)$ & $1(1)$ & $6(1)$ \\
\hline O1A1 & $60(2)$ & $29(1)$ & $48(2)$ & $5(1)$ & 11(1) & $15(1)$ \\
\hline C1A1 & $52(3)$ & $56(3)$ & $35(2)$ & $9(2)$ & $10(2)$ & $23(2)$ \\
\hline C2A1 & $65(5)$ & $37(3)$ & $39(3)$ & $1(2)$ & $6(4)$ & $2(3)$ \\
\hline C3A1 & $58(3)$ & $37(2)$ & $77(4)$ & $5(2)$ & $3(3)$ & $18(2)$ \\
\hline C4A1 & $47(3)$ & $38(3)$ & $62(3)$ & $-3(3)$ & $6(2)$ & $9(3)$ \\
\hline O1B1 & $37(2)$ & $48(2)$ & $44(2)$ & $22(2)$ & $14(2)$ & $16(2)$ \\
\hline C1B1 & $29(3)$ & $56(3)$ & $26(3)$ & $8(2)$ & $3(2)$ & $11(2)$ \\
\hline $\mathrm{C} 2 \mathrm{~B} 1$ & $30(3)$ & $41(4)$ & $32(3)$ & $6(3)$ & $0(2)$ & $2(3)$ \\
\hline C3B1 & $48(4)$ & $37(3)$ & $38(3)$ & $12(2)$ & $1(3)$ & $10(3)$ \\
\hline C4B1 & $31(3)$ & $30(3)$ & $30(3)$ & $6(3)$ & $0(3)$ & $5(3)$ \\
\hline
\end{tabular}




\subsection{Crystal Structure Determination of $\mathbf{3}_{\mathbf{P d}}$}

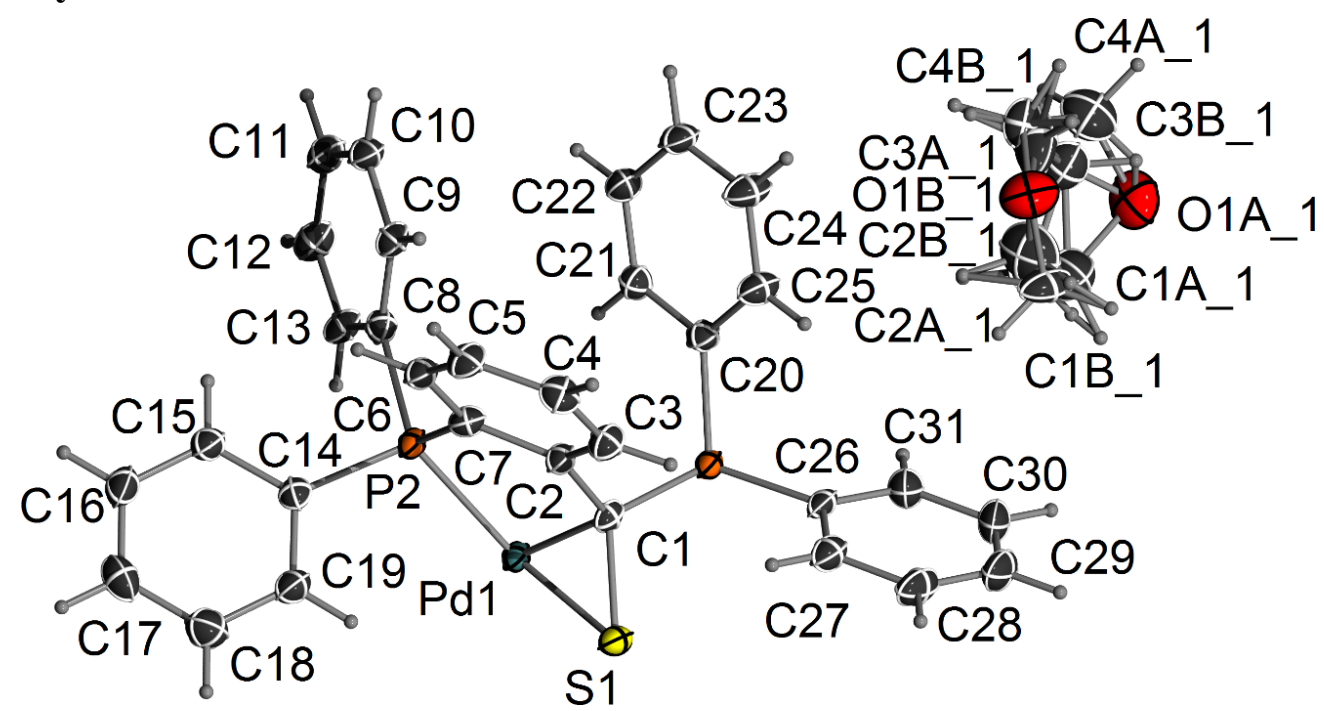

Figure S38. ORTEP Plot of compound 3Pd. Ellipsoids are drawn at the 50\% probability level.

Table S19. Atomic coordinates $\left(\times 10^{4}\right)$ and equivalent isotropic displacement parameters $\left(\AA^{2} \times 10^{3}\right)$ for compound $3_{\text {Pd. }} \mathrm{U}(\mathrm{eq})$ is defined as one third of the trace of the orthogonalized $\mathrm{U}_{\mathrm{ij}}$ tensor.

\begin{tabular}{|c|c|c|c|c|}
\hline & $\mathrm{x}$ & $\mathrm{y}$ & $\mathrm{z}$ & $\mathrm{U}(\mathrm{eq})$ \\
\hline $\operatorname{Pd}(1)$ & $4849(1)$ & $3850(1)$ & $-209(1)$ & $18(1)$ \\
\hline $\mathrm{S}(1)$ & 6534(1) & 3953(1) & $1146(1)$ & $22(1)$ \\
\hline $\mathrm{P}(1)$ & $4489(1)$ & $5248(1)$ & 1701(1) & $18(1)$ \\
\hline $\mathrm{C}(1)$ & $4762(3)$ & $3805(2)$ & $1362(2)$ & $18(1)$ \\
\hline $\mathrm{C}(6)$ & 1948(3) & $871(3)$ & $1314(2)$ & $23(1)$ \\
\hline$C(7)$ & $2804(3)$ & $1920(2)$ & $1008(2)$ & 21(1) \\
\hline $\mathrm{C}(2)$ & $3770(3)$ & $2697(2)$ & $1710(2)$ & 20(1) \\
\hline $\mathrm{P}(2)$ & 2803(1) & $2349(1)$ & $-303(1)$ & $18(1)$ \\
\hline $\mathrm{C}(3)$ & $3794(3)$ & $2419(3)$ & $2722(2)$ & $25(1)$ \\
\hline $\mathrm{C}(4)$ & 2937(4) & $1379(3)$ & $3020(2)$ & $27(1)$ \\
\hline $\mathrm{C}(5)$ & 2017(3) & $593(3)$ & $2311(2)$ & $27(1)$ \\
\hline $\mathrm{C}(8)$ & $1005(3)$ & $2382(2)$ & $-649(2)$ & $22(1)$ \\
\hline$C(10)$ & $-1473(3)$ & $2088(3)$ & $-356(3)$ & $28(1)$ \\
\hline $\mathrm{C}(9)$ & $-133(3)$ & $2051(2)$ & $-26(2)$ & $24(1)$ \\
\hline$C(12)$ & $-559(4)$ & $2775(3)$ & $-1936(3)$ & $30(1)$ \\
\hline$C(11)$ & $-1690(4)$ & $2440(3)$ & $-1313(3)$ & $31(1)$ \\
\hline$C(13)$ & $785(3)$ & $2762(3)$ & $-1607(2)$ & $25(1)$ \\
\hline$C(24)$ & 183(3) & $4360(3)$ & $1586(2)$ & $27(1)$ \\
\hline$C(23)$ & $-176(3)$ & $4300(3)$ & $2589(3)$ & $27(1)$ \\
\hline$C(14)$ & 2963(3) & $1052(2)$ & $-1034(2)$ & 21(1) \\
\hline$C(15)$ & 1861(3) & $301(3)$ & $-1649(2)$ & $25(1)$ \\
\hline$C(22)$ & $888(4)$ & $4561(3)$ & $3355(3)$ & $33(1)$ \\
\hline$C(16)$ & 2061(4) & $-670(3)$ & $-2173(3)$ & $31(1)$ \\
\hline $\mathrm{C}(21)$ & $2305(3)$ & $4857(3)$ & $3113(2)$ & $28(1)$ \\
\hline$C(17)$ & $3350(4)$ & $-917(3)$ & $-2105(3)$ & $35(1)$ \\
\hline$C(20)$ & $2673(3)$ & $4917(2)$ & $2102(2)$ & 21(1) \\
\hline$C(18)$ & $4459(4)$ & $-177(3)$ & $-1503(3)$ & $35(1)$ \\
\hline C(19) & $4267(3)$ & $796(3)$ & $-976(2)$ & $28(1)$ \\
\hline$C(31)$ & $5744(4)$ & $7060(3)$ & $3113(3)$ & $31(1)$ \\
\hline$C(30)$ & $6633(4)$ & $7629(3)$ & $3937(3)$ & $40(1)$ \\
\hline$C(29)$ & $7378(4)$ & 7034(4) & $4501(3)$ & $42(1)$ \\
\hline $\mathrm{C}(28)$ & $7244(4)$ & $5872(3)$ & $4240(2)$ & $35(1)$ \\
\hline
\end{tabular}




\begin{tabular}{lcccc}
\hline $\mathrm{C}(27)$ & $6347(3)$ & $5297(3)$ & $3418(2)$ & $27(1)$ \\
$\mathrm{C}(26)$ & $5588(3)$ & $5878(3)$ & $2852(2)$ & $22(1)$ \\
$\mathrm{C}(25)$ & $1590(3)$ & $4675(3)$ & $1342(2)$ & $23(1)$ \\
O1A1 & $2763(5)$ & $9589(3)$ & $4832(4)$ & $54(1)$ \\
C1A1 & $3502(12)$ & $8864(8)$ & $4337(8)$ & $61(3)$ \\
C2A1 & $3060(11)$ & $7742(7)$ & $4806(9)$ & $66(2)$ \\
C3A1 & $1558(15)$ & $7679(12)$ & $5120(15)$ & $63(5)$ \\
C4A1 & $1385(9)$ & $8853(7)$ & $4922(6)$ & $55(2)$ \\
O1B1 & $2285(10)$ & $7058(8)$ & $5516(7)$ & $46(3)$ \\
C1B1 & $3598(14)$ & $7620(15)$ & $5109(10)$ & $39(4)$ \\
C2B1 & $3277(17)$ & $8311(19)$ & $4272(10)$ & $40(4)$ \\
C3B1 & $1974(16)$ & $8623(12)$ & $4670(12)$ & $45(4)$ \\
C4B1 & $1230(20)$ & $7498(19)$ & $5110(20)$ & $29(5)$ \\
\hline
\end{tabular}

Table S20. Anisotropic displacement parameters $\left(\AA^{2} \times 10^{3}\right)$ for compound 3Pd. The anisotropic displacement factor exponent takes the form: $-2 \pi^{2}\left[\mathrm{~h}^{2} \mathrm{a}^{* 2} \mathrm{U}^{11}+\ldots+2 \mathrm{~h} \mathrm{k} \mathrm{a}^{*} \mathrm{~b}^{*} \mathrm{U}^{12}\right]$.

\begin{tabular}{|c|c|c|c|c|c|c|}
\hline & $\mathrm{U}^{11}$ & $\mathrm{U}^{22}$ & $\mathrm{U}^{33}$ & $\mathrm{U}^{23}$ & $\mathrm{U}^{13}$ & $\mathrm{U}^{12}$ \\
\hline $\operatorname{Pd}(1)$ & $15(1)$ & $17(1)$ & $18(1)$ & 1(1) & $0(1)$ & $0(1)$ \\
\hline $\mathrm{S}(1)$ & $15(1)$ & $26(1)$ & $24(1)$ & $2(1)$ & $0(1)$ & $3(1)$ \\
\hline $\mathrm{P}(1)$ & $15(1)$ & $18(1)$ & 18(1) & $0(1)$ & $0(1)$ & 1(1) \\
\hline $\mathrm{C}(1)$ & $17(1)$ & $21(1)$ & $13(1)$ & $2(1)$ & $-2(1)$ & $2(1)$ \\
\hline$C(6)$ & $17(1)$ & $22(1)$ & $28(2)$ & 2(1) & 1(1) & $3(1)$ \\
\hline$C(7)$ & $16(1)$ & $22(1)$ & $23(1)$ & $4(1)$ & 1(1) & $4(1)$ \\
\hline $\mathrm{C}(2)$ & $16(1)$ & $18(1)$ & $24(1)$ & $0(1)$ & $-1(1)$ & $2(1)$ \\
\hline $\mathrm{P}(2)$ & $15(1)$ & $17(1)$ & $20(1)$ & 1(1) & $0(1)$ & $0(1)$ \\
\hline $\mathrm{C}(3)$ & $24(2)$ & $25(1)$ & $23(1)$ & $2(1)$ & $0(1)$ & $4(1)$ \\
\hline $\mathrm{C}(4)$ & $29(2)$ & $29(2)$ & $23(1)$ & $6(1)$ & $4(1)$ & $9(1)$ \\
\hline$C(5)$ & $24(2)$ & $21(1)$ & $32(2)$ & $5(1)$ & $6(1)$ & 1(1) \\
\hline $\mathrm{C}(8)$ & $21(2)$ & $17(1)$ & $25(1)$ & $-1(1)$ & $-1(1)$ & $4(1)$ \\
\hline$C(10)$ & $18(2)$ & $23(1)$ & $40(2)$ & $-2(1)$ & $3(1)$ & $3(1)$ \\
\hline $\mathrm{C}(9)$ & $21(2)$ & $20(1)$ & $28(2)$ & $1(1)$ & 1(1) & 1(1) \\
\hline$C(12)$ & $27(2)$ & $25(1)$ & $36(2)$ & $5(1)$ & $-5(1)$ & $4(1)$ \\
\hline $\mathrm{C}(11)$ & $23(2)$ & $22(1)$ & $45(2)$ & $1(1)$ & $-5(1)$ & $6(1)$ \\
\hline$C(13)$ & $20(2)$ & $23(1)$ & $30(2)$ & $3(1)$ & $1(1)$ & $0(1)$ \\
\hline $\mathrm{C}(24)$ & $19(2)$ & $29(2)$ & $32(2)$ & $-4(1)$ & $-3(1)$ & $6(1)$ \\
\hline $\mathrm{C}(23)$ & $15(2)$ & $30(2)$ & $35(2)$ & $3(1)$ & $3(1)$ & $2(1)$ \\
\hline $\mathrm{C}(14)$ & $22(2)$ & $17(1)$ & $20(1)$ & $2(1)$ & $5(1)$ & 1(1) \\
\hline $\mathrm{C}(15)$ & $20(2)$ & $27(1)$ & $26(2)$ & $1(1)$ & 1(1) & $2(1)$ \\
\hline $\mathrm{C}(22)$ & $21(2)$ & $46(2)$ & $31(2)$ & $10(1)$ & $4(1)$ & $4(1)$ \\
\hline$C(16)$ & $29(2)$ & $26(2)$ & $30(2)$ & $-4(1)$ & $-1(1)$ & $-1(1)$ \\
\hline $\mathrm{C}(21)$ & $18(2)$ & $38(2)$ & $24(2)$ & $7(1)$ & $0(1)$ & $4(1)$ \\
\hline $\mathrm{C}(17)$ & $46(2)$ & $27(2)$ & $34(2)$ & $-2(1)$ & $6(2)$ & $13(2)$ \\
\hline $\mathrm{C}(20)$ & $17(1)$ & $18(1)$ & $25(1)$ & $2(1)$ & $2(1)$ & $3(1)$ \\
\hline$C(18)$ & $33(2)$ & $39(2)$ & $36(2)$ & $2(1)$ & $3(1)$ & $15(2)$ \\
\hline$C(19)$ & $20(2)$ & $28(2)$ & $32(2)$ & $0(1)$ & $-3(1)$ & $3(1)$ \\
\hline $\mathrm{C}(31)$ & $29(2)$ & $32(2)$ & $30(2)$ & $-6(1)$ & $2(1)$ & $5(1)$ \\
\hline $\mathrm{C}(30)$ & $33(2)$ & $40(2)$ & $37(2)$ & $-18(2)$ & $0(2)$ & $0(2)$ \\
\hline $\mathrm{C}(29)$ & $27(2)$ & $62(2)$ & $28(2)$ & $-16(2)$ & $-6(1)$ & $2(2)$ \\
\hline $\mathrm{C}(28)$ & $24(2)$ & $55(2)$ & $23(2)$ & $0(1)$ & $-5(1)$ & $6(2)$ \\
\hline $\mathrm{C}(27)$ & $21(2)$ & $34(2)$ & $23(2)$ & $2(1)$ & 1(1) & 1(1) \\
\hline$C(26)$ & $14(1)$ & $28(1)$ & $19(1)$ & $-2(1)$ & $-1(1)$ & $-3(1)$ \\
\hline $\mathrm{C}(25)$ & $21(2)$ & $26(1)$ & 21(1) & $0(1)$ & $0(1)$ & $5(1)$ \\
\hline O1A1 & $65(3)$ & $34(2)$ & $60(3)$ & $10(2)$ & $11(2)$ & $10(2)$ \\
\hline C1A1 & $53(5)$ & $52(5)$ & $76(6)$ & $2(5)$ & $11(4)$ & $10(5)$ \\
\hline
\end{tabular}




\begin{tabular}{lllllll}
\hline C2A1 & $75(7)$ & $48(4)$ & $80(7)$ & $14(5)$ & $14(5)$ & $22(5)$ \\
C3A1 & $83(9)$ & $39(5)$ & $48(6)$ & $-2(4)$ & $14(6)$ & $-12(5)$ \\
C4A1 & $62(5)$ & $60(4)$ & $47(4)$ & $12(3)$ & $12(3)$ & $22(4)$ \\
O1B1 & $41(5)$ & $49(5)$ & $49(5)$ & $20(4)$ & $4(4)$ & $12(4)$ \\
C1B1 & $25(7)$ & $61(9)$ & $29(7)$ & $14(6)$ & $7(5)$ & $6(6)$ \\
C2B1 & $40(9)$ & $59(12)$ & $22(6)$ & $4(9)$ & $-2(6)$ & $17(10)$ \\
C3B1 & $44(9)$ & $39(8)$ & $51(9)$ & $5(6)$ & $-6(7)$ & $12(7)$ \\
C4B1 & $40(9)$ & $22(8)$ & $23(8)$ & $7(7)$ & $-6(6)$ & $7(8)$ \\
\hline
\end{tabular}

\subsection{Crystal Structure Determination of 4}

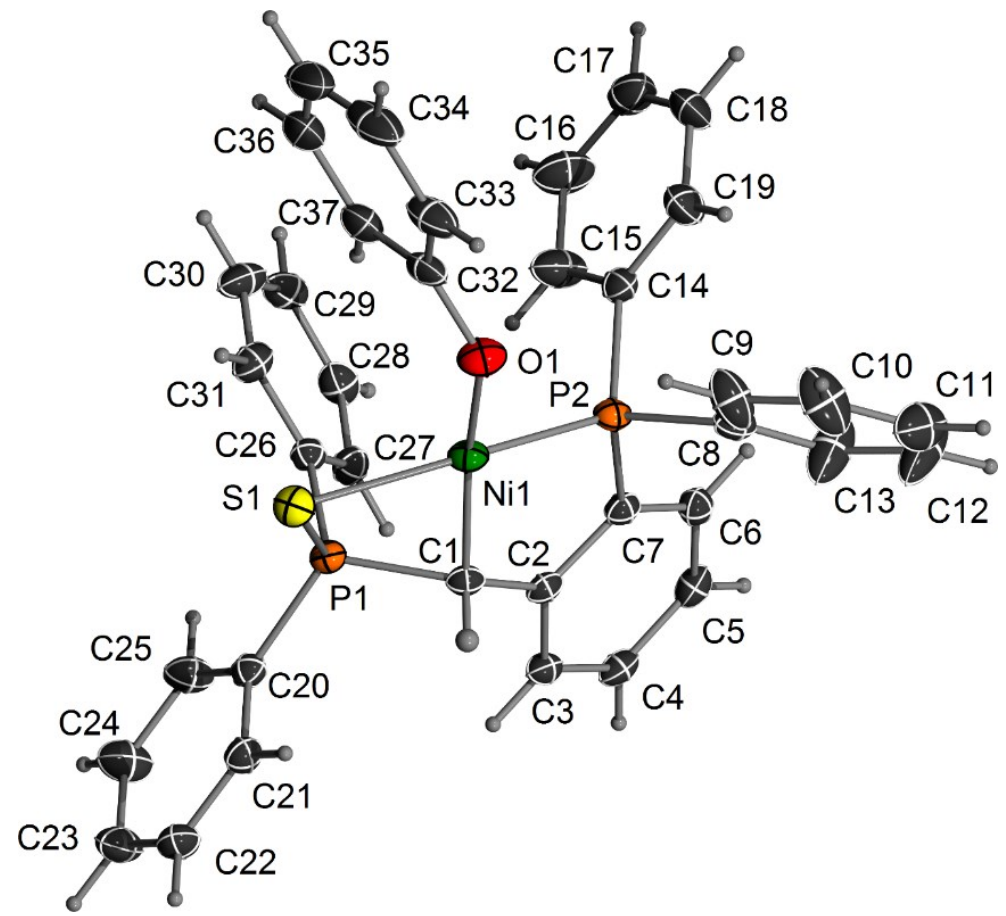

Figure S39. ORTEP Plot of compound 4. Ellipsoids are drawn at the 50\% probability level.

Table S21. Atomic coordinates ( x 10 $)$ and equivalent isotropic displacement parameters $\left(\AA^{2} \times 10^{3}\right)$ for compound 4. $U(\mathrm{eq})$ is defined as one third of the trace of the orthogonalized $U_{\mathrm{ij}}$ tensor.

\begin{tabular}{ccccc}
\hline & $\mathrm{x}$ & $\mathrm{y}$ & $\mathrm{z}$ & $\mathrm{U}(\mathrm{eq})$ \\
\hline $\mathrm{Ni}(1)$ & $3353(1)$ & $5453(1)$ & $3473(1)$ & $20(1)$ \\
$\mathrm{C}(1)$ & $3266(1)$ & $4312(1)$ & $2996(1)$ & $18(1)$ \\
$\mathrm{O}(1)$ & $3213(1)$ & $6549(1)$ & $3910(1)$ & $29(1)$ \\
$\mathrm{S}(1)$ & $3445(1)$ & $7024(1)$ & $2990(1)$ & $24(1)$ \\
$\mathrm{P}(1)$ & $3840(1)$ & $5406(1)$ & $2767(1)$ & $17(1)$ \\
$\mathrm{P}(2)$ & $3541(1)$ & $3713(1)$ & $3821(1)$ & $20(1)$ \\
$\mathrm{C}(2)$ & $3454(1)$ & $2903(1)$ & $3052(1)$ & $18(1)$ \\
$\mathrm{C}(6)$ & $3842(1)$ & $1170(1)$ & $3530(1)$ & $22(1)$ \\
$\mathrm{C}(5)$ & $3860(1)$ & $324(1)$ & $3209(1)$ & $23(1)$ \\
$\mathrm{C}(4)$ & $3665(1)$ & $751(1)$ & $2811(1)$ & $22(1)$ \\
$\mathrm{C}(3)$ & $3465(1)$ & $2033(1)$ & $2731(1)$ & $20(1)$ \\
$\mathrm{C}(7)$ & $3624(1)$ & $2455(1)$ & $3450(1)$ & $19(1)$ \\
$\mathrm{C}(9)$ & $2412(1)$ & $4184(2)$ & $4282(1)$ & $40(1)$ \\
$\mathrm{C}(8)$ & $2813(1)$ & $3231(2)$ & $4116(1)$ & $25(1)$ \\
$\mathrm{C}(10)$ & $1874(1)$ & $3843(2)$ & $4521(1)$ & $50(1)$ \\
\hline
\end{tabular}




\begin{tabular}{lllll}
\hline $\mathrm{C}(12)$ & $2127(1)$ & $1622(2)$ & $4432(1)$ & $63(1)$ \\
$\mathrm{C}(11)$ & $1732(1)$ & $2567(2)$ & $4595(1)$ & $44(1)$ \\
$\mathrm{C}(13)$ & $2656(1)$ & $1952(2)$ & $4187(1)$ & $53(1)$ \\
$\mathrm{C}(15)$ & $5074(1)$ & $3456(2)$ & $4020(1)$ & $40(1)$ \\
$\mathrm{C}(16)$ & $5741(1)$ & $3301(2)$ & $4276(1)$ & $45(1)$ \\
$\mathrm{C}(17)$ & $5744(1)$ & $3254(2)$ & $4691(1)$ & $43(1)$ \\
$\mathrm{C}(18)$ & $5090(1)$ & $3403(2)$ & $4848(1)$ & $50(1)$ \\
$\mathrm{C}(19)$ & $4417(1)$ & $3576(2)$ & $4591(1)$ & $37(1)$ \\
$\mathrm{C}(20)$ & $3733(1)$ & $5402(1)$ & $2219(1)$ & $20(1)$ \\
$\mathrm{C}(21)$ & $3028(1)$ & $5725(1)$ & $2012(1)$ & $23(1)$ \\
$\mathrm{C}(22)$ & $2913(1)$ & $5837(2)$ & $1593(1)$ & $28(1)$ \\
$\mathrm{C}(23)$ & $3500(1)$ & $5619(2)$ & $1377(1)$ & $34(1)$ \\
$\mathrm{C}(24)$ & $4199(1)$ & $5282(2)$ & $1579(1)$ & $38(1)$ \\
$\mathrm{C}(25)$ & $4319(1)$ & $5171(2)$ & $1998(1)$ & $29(1)$ \\
$\mathrm{C}(26)$ & $4820(1)$ & $5138(1)$ & $2951(1)$ & $20(1)$ \\
$\mathrm{C}(27)$ & $5153(1)$ & $3931(1)$ & $2908(1)$ & $23(1)$ \\
$\mathrm{C}(28)$ & $5897(1)$ & $3734(2)$ & $3072(1)$ & $26(1)$ \\
$\mathrm{C}(29)$ & $6309(1)$ & $4731(2)$ & $3275(1)$ & $30(1)$ \\
$\mathrm{C}(30)$ & $5987(1)$ & $5936(2)$ & $3309(1)$ & $32(1)$ \\
$\mathrm{C}(31)$ & $5245(1)$ & $6143(2)$ & $3147(1)$ & $26(1)$ \\
$\mathrm{C}(32)$ & $3675(1)$ & $7441(1)$ & $4083(1)$ & $27(1)$ \\
$\mathrm{C}(33)$ & $3415(1)$ & $8342(2)$ & $4350(1)$ & $37(1)$ \\
$\mathrm{C}(34)$ & $3888(2)$ & $9303(2)$ & $4538(1)$ & $53(1)$ \\
$\mathrm{C}(35)$ & $4614(2)$ & $9411(2)$ & $4466(1)$ & $59(1)$ \\
$\mathrm{C}(36)$ & $4875(1)$ & $8531(2)$ & $4209(1)$ & $49(1)$ \\
$\mathrm{C}(37)$ & $4420(1)$ & $7549(2)$ & $4021(1)$ & $34(1)$ \\
$\mathrm{C}(14)$ & $4405(1)$ & $3581(1)$ & $4176(1)$ & $23(1)$ \\
\hline
\end{tabular}

Table S22. Anisotropic displacement parameters $\left(\AA^{2} \times 10^{3}\right)$ for compound 4. The anisotropic displacement factor exponent takes the form: $-2 \pi^{2}\left[\mathrm{~h}^{2} \mathrm{a}^{* 2} \mathrm{U}^{11}+\ldots+2 \mathrm{~h} \mathrm{k} \mathrm{a}^{*} \mathrm{~b}^{*} \mathrm{U}^{12}\right]$.

\begin{tabular}{ccccccc}
\hline & $\mathrm{U}^{11}$ & $\mathrm{U}^{22}$ & $\mathrm{U}^{33}$ & $\mathrm{U}^{23}$ & $\mathrm{U}^{13}$ & $\mathrm{U}^{12}$ \\
\hline $\mathrm{Ni}(1)$ & $22(1)$ & $18(1)$ & $20(1)$ & $-5(1)$ & $2(1)$ & $3(1)$ \\
$\mathrm{C}(1)$ & $16(1)$ & $20(1)$ & $19(1)$ & $-3(1)$ & $1(1)$ & $1(1)$ \\
$\mathrm{O}(1)$ & $32(1)$ & $26(1)$ & $28(1)$ & $-10(1)$ & $5(1)$ & $2(1)$ \\
$\mathrm{S}(1)$ & $28(1)$ & $17(1)$ & $26(1)$ & $-2(1)$ & $3(1)$ & $5(1)$ \\
$\mathrm{P}(1)$ & $17(1)$ & $16(1)$ & $19(1)$ & $-2(1)$ & $1(1)$ & $2(1)$ \\
$\mathrm{P}(2)$ & $21(1)$ & $21(1)$ & $17(1)$ & $-3(1)$ & $3(1)$ & $0(1)$ \\
$\mathrm{C}(2)$ & $13(1)$ & $20(1)$ & $22(1)$ & $-3(1)$ & $3(1)$ & $-2(1)$ \\
$\mathrm{C}(6)$ & $21(1)$ & $23(1)$ & $24(1)$ & $1(1)$ & $5(1)$ & $-2(1)$ \\
$\mathrm{C}(5)$ & $20(1)$ & $18(1)$ & $33(1)$ & $-1(1)$ & $6(1)$ & $-2(1)$ \\
$\mathrm{C}(4)$ & $19(1)$ & $21(1)$ & $27(1)$ & $-8(1)$ & $5(1)$ & $-4(1)$ \\
$\mathrm{C}(3)$ & $18(1)$ & $24(1)$ & $20(1)$ & $-4(1)$ & $2(1)$ & $-3(1)$ \\
$\mathrm{C}(7)$ & $16(1)$ & $21(1)$ & $20(1)$ & $-3(1)$ & $4(1)$ & $-2(1)$ \\
$\mathrm{C}(9)$ & $47(1)$ & $41(1)$ & $36(1)$ & $11(1)$ & $20(1)$ & $17(1)$ \\
$\mathrm{C}(8)$ & $23(1)$ & $35(1)$ & $18(1)$ & $-6(1)$ & $4(1)$ & $-2(1)$ \\
$\mathrm{C}(10)$ & $51(1)$ & $60(1)$ & $44(1)$ & $12(1)$ & $29(1)$ & $24(1)$ \\
$\mathrm{C}(12)$ & $80(2)$ & $49(1)$ & $73(1)$ & $-32(1)$ & $54(1)$ & $-37(1)$ \\
$\mathrm{C}(11)$ & $34(1)$ & $68(1)$ & $35(1)$ & $-11(1)$ & $17(1)$ & $-12(1)$ \\
$\mathrm{C}(13)$ & $69(1)$ & $37(1)$ & $65(1)$ & $-25(1)$ & $48(1)$ & $-21(1)$ \\
$\mathrm{C}(15)$ & $27(1)$ & $62(1)$ & $29(1)$ & $-10(1)$ & $1(1)$ & $-3(1)$ \\
$\mathrm{C}(16)$ & $27(1)$ & $58(1)$ & $49(1)$ & $-9(1)$ & $-3(1)$ & $0(1)$ \\
$\mathrm{C}(17)$ & $36(1)$ & $37(1)$ & $50(1)$ & $13(1)$ & $-16(1)$ & $-8(1)$ \\
$\mathrm{C}(18)$ & $50(1)$ & $70(1)$ & $26(1)$ & $20(1)$ & $-8(1)$ & $-19(1)$ \\
$\mathrm{C}(19)$ & $35(1)$ & $53(1)$ & $23(1)$ & $7(1)$ & $2(1)$ & $-9(1)$ \\
\hline
\end{tabular}




\begin{tabular}{lllllll}
\hline $\mathrm{C}(20)$ & $20(1)$ & $17(1)$ & $22(1)$ & $-1(1)$ & $2(1)$ & $-1(1)$ \\
$\mathrm{C}(21)$ & $18(1)$ & $25(1)$ & $26(1)$ & $1(1)$ & $3(1)$ & $-1(1)$ \\
$\mathrm{C}(22)$ & $22(1)$ & $35(1)$ & $27(1)$ & $1(1)$ & $-2(1)$ & $-3(1)$ \\
$\mathrm{C}(23)$ & $30(1)$ & $50(1)$ & $20(1)$ & $-3(1)$ & $1(1)$ & $-5(1)$ \\
$\mathrm{C}(24)$ & $26(1)$ & $60(1)$ & $27(1)$ & $-9(1)$ & $7(1)$ & $2(1)$ \\
$\mathrm{C}(25)$ & $20(1)$ & $41(1)$ & $26(1)$ & $-5(1)$ & $1(1)$ & $4(1)$ \\
$\mathrm{C}(26)$ & $17(1)$ & $23(1)$ & $19(1)$ & $1(1)$ & $2(1)$ & $0(1)$ \\
$\mathrm{C}(27)$ & $20(1)$ & $22(1)$ & $26(1)$ & $1(1)$ & $3(1)$ & $-1(1)$ \\
$\mathrm{C}(28)$ & $21(1)$ & $31(1)$ & $28(1)$ & $6(1)$ & $5(1)$ & $6(1)$ \\
$\mathrm{C}(29)$ & $17(1)$ & $48(1)$ & $24(1)$ & $2(1)$ & $2(1)$ & $1(1)$ \\
$\mathrm{C}(30)$ & $23(1)$ & $43(1)$ & $30(1)$ & $-11(1)$ & $3(1)$ & $-9(1)$ \\
$\mathrm{C}(31)$ & $24(1)$ & $26(1)$ & $28(1)$ & $-6(1)$ & $5(1)$ & $-3(1)$ \\
$\mathrm{C}(32)$ & $40(1)$ & $22(1)$ & $16(1)$ & $2(1)$ & $-4(1)$ & $4(1)$ \\
$\mathrm{C}(33)$ & $61(1)$ & $27(1)$ & $21(1)$ & $-3(1)$ & $-3(1)$ & $9(1)$ \\
$\mathrm{C}(34)$ & $102(2)$ & $28(1)$ & $24(1)$ & $-5(1)$ & $-8(1)$ & $1(1)$ \\
$\mathrm{C}(35)$ & $95(2)$ & $43(1)$ & $31(1)$ & $7(1)$ & $-20(1)$ & $-29(1)$ \\
$\mathrm{C}(36)$ & $56(1)$ & $56(1)$ & $31(1)$ & $17(1)$ & $-14(1)$ & $-22(1)$ \\
$\mathrm{C}(37)$ & $40(1)$ & $37(1)$ & $22(1)$ & $7(1)$ & $-5(1)$ & $-3(1)$ \\
$\mathrm{C}(14)$ & $26(1)$ & $20(1)$ & $22(1)$ & $0(1)$ & $0(1)$ & $-2(1)$ \\
\hline
\end{tabular}

\subsection{Crystal Structure Determination of 5}

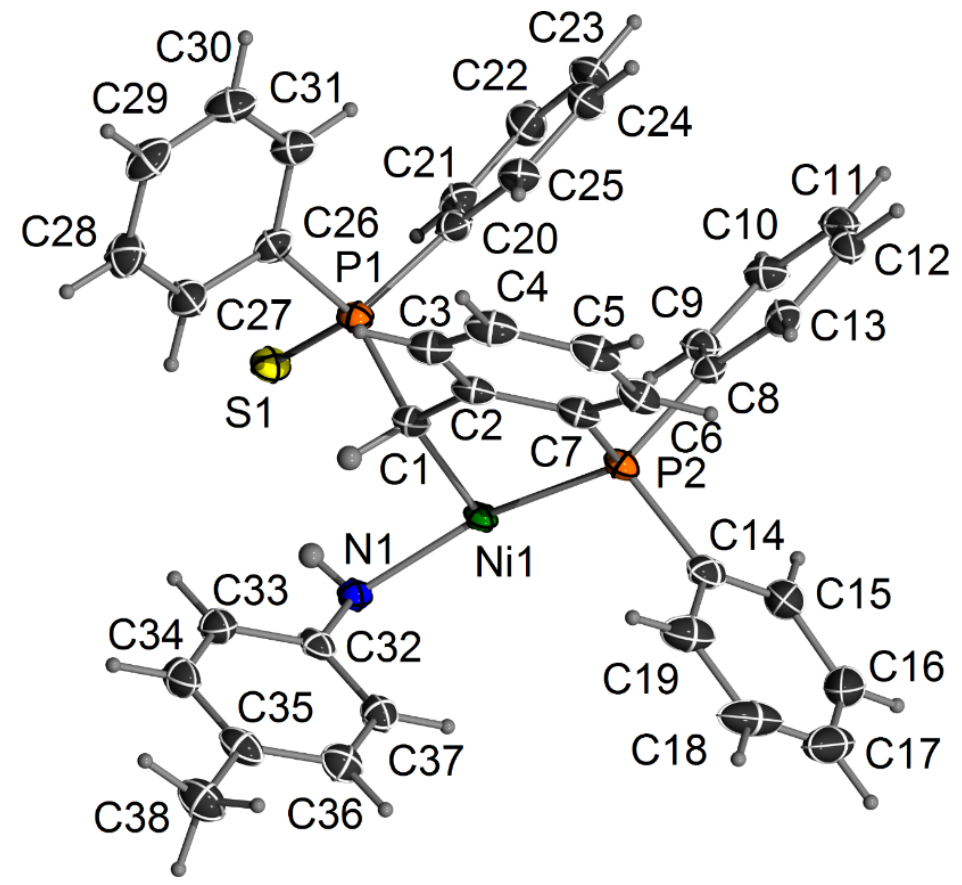

Figure S40. ORTEP Plot of compound $\mathbf{5}_{2}$. Ellipsoids are drawn at the 50\% probability level. 
Table S23. Atomic coordinates $\left(\times 10^{4}\right)$ and equivalent isotropic displacement parameters $\left(\AA^{2} \times 10^{3}\right)$ for compound 52. $\mathrm{U}(\mathrm{eq})$ is defined as one third of the trace of the orthogonalized $\mathrm{U}_{\mathrm{ij}}$ tensor.

\begin{tabular}{|c|c|c|c|c|}
\hline & $\mathrm{X}$ & $\mathrm{y}$ & $\mathrm{Z}$ & $\mathrm{U}(\mathrm{eq})$ \\
\hline $\mathrm{Ni}(1)$ & $4007(1)$ & $4759(1)$ & $4164(1)$ & $17(1)$ \\
\hline $\mathrm{S}(1)$ & $3433(1)$ & $6913(1)$ & $3461(1)$ & $23(1)$ \\
\hline $\mathrm{P}(1)$ & $2557(1)$ & $6016(1)$ & $2572(1)$ & $18(1)$ \\
\hline $\mathrm{N}(1)$ & $5225(1)$ & $5431(1)$ & $4400(1)$ & $19(1)$ \\
\hline$C(1)$ & $3264(1)$ & $5046(1)$ & $2728(1)$ & $19(1)$ \\
\hline $\mathrm{C}(2)$ & $2653(1)$ & $4336(1)$ & $2038(1)$ & $20(1)$ \\
\hline$P(2)$ & $2937(1)$ & $3736(1)$ & $3787(1)$ & $19(1)$ \\
\hline $\mathrm{C}(3)$ & $2315(1)$ & $4337(1)$ & $1008(1)$ & $25(1)$ \\
\hline $\mathrm{C}(4)$ & $1828(1)$ & $3640(1)$ & $436(1)$ & $28(1)$ \\
\hline $\mathrm{C}(5)$ & 1688(1) & 2922(1) & $871(1)$ & $29(1)$ \\
\hline$C(6)$ & $2016(1)$ & $2905(1)$ & $1887(1)$ & $26(1)$ \\
\hline $\mathrm{C}(8)$ & 1774(1) & $3708(1)$ & 3958(1) & $20(1)$ \\
\hline $\mathrm{C}(7)$ & $2477(1)$ & $3619(1)$ & $2460(1)$ & 21(1) \\
\hline $\mathrm{C}(9)$ & $1686(1)$ & $4278(1)$ & 4601(1) & $24(1)$ \\
\hline$C(10)$ & $814(1)$ & $4265(1)$ & $4753(1)$ & $27(1)$ \\
\hline $\mathrm{C}(11)$ & $19(1)$ & $3699(1)$ & $4250(1)$ & $27(1)$ \\
\hline$C(12)$ & $94(1)$ & $3130(1)$ & $3598(1)$ & $25(1)$ \\
\hline $\mathrm{C}(13)$ & $970(1)$ & $3128(1)$ & $3458(1)$ & $24(1)$ \\
\hline$C(14)$ & $3609(1)$ & $2763(1)$ & $4358(1)$ & $26(1)$ \\
\hline$C(15)$ & $3651(1)$ & 2489(1) & $5243(1)$ & $34(1)$ \\
\hline $\mathrm{C}(17)$ & $4752(2)$ & $1342(1)$ & $5331(2)$ & $48(1)$ \\
\hline $\mathrm{C}(18)$ & $4725(1)$ & $1615(1)$ & $4461(2)$ & $46(1)$ \\
\hline$C(19)$ & $4170(1)$ & $2330(1)$ & $3976(1)$ & $36(1)$ \\
\hline $\mathrm{C}(20)$ & $1386(1)$ & $5838(1)$ & $2694(1)$ & 21(1) \\
\hline $\mathrm{C}(21)$ & $1234(1)$ & $6329(1)$ & $3368(1)$ & $25(1)$ \\
\hline $\mathrm{C}(22)$ & $308(1)$ & $6276(1)$ & $3423(1)$ & $29(1)$ \\
\hline $\mathrm{C}(23)$ & $-461(1)$ & $5728(1)$ & 2819(1) & $30(1)$ \\
\hline $\mathrm{C}(24)$ & $-308(1)$ & $5216(1)$ & $2164(1)$ & $28(1)$ \\
\hline$C(25)$ & $610(1)$ & $5268(1)$ & 2098(1) & $24(1)$ \\
\hline$C(26)$ & $2097(1)$ & $6338(1)$ & $1278(1)$ & $22(1)$ \\
\hline $\mathrm{C}(27)$ & $2867(1)$ & $6563(1)$ & $1024(1)$ & $28(1)$ \\
\hline $\mathrm{C}(28)$ & $2612(1)$ & $6745(1)$ & $45(1)$ & $31(1)$ \\
\hline C(29) & $1586(1)$ & $6703(1)$ & $-692(1)$ & $31(1)$ \\
\hline$C(30)$ & $810(1)$ & $6511(1)$ & $-446(1)$ & $31(1)$ \\
\hline $\mathrm{C}(31)$ & 1061(1) & $6329(1)$ & $536(1)$ & $26(1)$ \\
\hline$C(32)$ & $5737(1)$ & $5136(1)$ & $3868(1)$ & $20(1)$ \\
\hline $\mathrm{C}(33)$ & $5892(1)$ & $5646(1)$ & $3203(1)$ & $26(1)$ \\
\hline$C(34)$ & $6421(1)$ & $5348(1)$ & $2710(1)$ & $29(1)$ \\
\hline $\mathrm{C}(35)$ & $6796(1)$ & $4538(1)$ & $2837(1)$ & $27(1)$ \\
\hline$C(36)$ & $6626(1)$ & $4023(1)$ & $3492(1)$ & $26(1)$ \\
\hline $\mathrm{C}(37)$ & $6110(1)$ & 4311(1) & 3999(1) & $23(1)$ \\
\hline $\mathrm{C}(38)$ & $7351(1)$ & $4218(1)$ & $2285(1)$ & $34(1)$ \\
\hline$C(16)$ & $4234(2)$ & $1785(1)$ & $5736(2)$ & $45(1)$ \\
\hline
\end{tabular}


Table S24. Anisotropic displacement parameters $\left(\AA^{2} \times 10^{3}\right)$ for compound $\mathbf{5}_{2}$. The anisotropic displacement factor exponent takes the form: $-2 \pi^{2}\left[\mathrm{~h}^{2} \mathrm{a}^{* 2} \mathrm{U}^{11}+\ldots+2 \mathrm{~h} \mathrm{k} \mathrm{a}^{*} \mathrm{~b}^{*} \mathrm{U}^{12}\right]$.

\begin{tabular}{|c|c|c|c|c|c|c|}
\hline & $\mathrm{U}^{11}$ & $\mathrm{U}^{22}$ & $\mathrm{U}^{33}$ & $\mathrm{U}^{23}$ & $\mathrm{U}^{13}$ & $\mathrm{U}^{12}$ \\
\hline $\mathrm{Ni}(1)$ & $13(1)$ & $22(1)$ & $13(1)$ & $-1(1)$ & $4(1)$ & $-2(1)$ \\
\hline $\mathrm{S}(1)$ & $21(1)$ & $23(1)$ & $22(1)$ & $-3(1)$ & $7(1)$ & $-1(1)$ \\
\hline $\mathrm{P}(1)$ & $16(1)$ & $22(1)$ & $15(1)$ & $0(1)$ & $6(1)$ & $1(1)$ \\
\hline $\mathrm{N}(1)$ & $17(1)$ & 21(1) & $17(1)$ & $0(1)$ & $6(1)$ & $-1(1)$ \\
\hline $\mathrm{C}(1)$ & $16(1)$ & $23(1)$ & $17(1)$ & $-1(1)$ & $7(1)$ & $1(1)$ \\
\hline $\mathrm{C}(2)$ & $13(1)$ & $28(1)$ & $18(1)$ & $-4(1)$ & $5(1)$ & $4(1)$ \\
\hline $\mathrm{P}(2)$ & $15(1)$ & $22(1)$ & $18(1)$ & $-2(1)$ & $6(1)$ & $-2(1)$ \\
\hline $\mathrm{C}(3)$ & $20(1)$ & $32(1)$ & $20(1)$ & $-3(1)$ & $8(1)$ & $4(1)$ \\
\hline $\mathrm{C}(4)$ & $22(1)$ & $40(1)$ & $19(1)$ & $-8(1)$ & $6(1)$ & $4(1)$ \\
\hline $\mathrm{C}(5)$ & $22(1)$ & $35(1)$ & $27(1)$ & $-14(1)$ & $9(1)$ & $-3(1)$ \\
\hline $\mathrm{C}(6)$ & $20(1)$ & $30(1)$ & $28(1)$ & $-8(1)$ & $10(1)$ & $-3(1)$ \\
\hline $\mathrm{C}(8)$ & $16(1)$ & $25(1)$ & $17(1)$ & $5(1)$ & $5(1)$ & $1(1)$ \\
\hline$C(7)$ & $15(1)$ & $28(1)$ & $20(1)$ & $-6(1)$ & $6(1)$ & $0(1)$ \\
\hline $\mathrm{C}(9)$ & $17(1)$ & $25(1)$ & $24(1)$ & $0(1)$ & $6(1)$ & $1(1)$ \\
\hline $\mathrm{C}(10)$ & $22(1)$ & $32(1)$ & $27(1)$ & $1(1)$ & 11(1) & $5(1)$ \\
\hline $\mathrm{C}(11)$ & $18(1)$ & $38(1)$ & $23(1)$ & $9(1)$ & $9(1)$ & $4(1)$ \\
\hline $\mathrm{C}(12)$ & $18(1)$ & $34(1)$ & $18(1)$ & $5(1)$ & $4(1)$ & $-5(1)$ \\
\hline $\mathrm{C}(13)$ & $24(1)$ & $28(1)$ & $16(1)$ & $1(1)$ & $7(1)$ & $-4(1)$ \\
\hline $\mathrm{C}(14)$ & $16(1)$ & $23(1)$ & $31(1)$ & $-3(1)$ & $5(1)$ & $-3(1)$ \\
\hline$C(15)$ & $24(1)$ & $34(1)$ & $42(1)$ & $9(1)$ & $13(1)$ & $-1(1)$ \\
\hline$C(17)$ & $26(1)$ & $31(1)$ & $65(2)$ & $6(1)$ & $3(1)$ & $2(1)$ \\
\hline$C(18)$ & $23(1)$ & $39(1)$ & $56(1)$ & $-15(1)$ & $2(1)$ & $7(1)$ \\
\hline C(19) & $22(1)$ & $38(1)$ & $37(1)$ & $-8(1)$ & $6(1)$ & $2(1)$ \\
\hline$C(20)$ & $19(1)$ & $24(1)$ & $19(1)$ & $4(1)$ & $8(1)$ & $4(1)$ \\
\hline $\mathrm{C}(21)$ & $26(1)$ & $26(1)$ & $22(1)$ & 1(1) & $11(1)$ & $2(1)$ \\
\hline$C(22)$ & $32(1)$ & $30(1)$ & $30(1)$ & 1(1) & 21(1) & $4(1)$ \\
\hline$C(23)$ & $25(1)$ & $33(1)$ & $37(1)$ & $6(1)$ & $19(1)$ & $5(1)$ \\
\hline $\mathrm{C}(24)$ & $21(1)$ & $29(1)$ & $32(1)$ & $0(1)$ & $11(1)$ & $-1(1)$ \\
\hline$C(25)$ & $22(1)$ & $27(1)$ & $24(1)$ & $-1(1)$ & $10(1)$ & $2(1)$ \\
\hline$C(26)$ & $25(1)$ & $23(1)$ & $17(1)$ & $1(1)$ & $9(1)$ & $3(1)$ \\
\hline$C(27)$ & $25(1)$ & $35(1)$ & $23(1)$ & $4(1)$ & $10(1)$ & $-1(1)$ \\
\hline$C(28)$ & $35(1)$ & $36(1)$ & $27(1)$ & $6(1)$ & $18(1)$ & $3(1)$ \\
\hline C(29) & $40(1)$ & $31(1)$ & $20(1)$ & $5(1)$ & $14(1)$ & $7(1)$ \\
\hline$C(30)$ & $28(1)$ & $37(1)$ & $21(1)$ & $2(1)$ & $6(1)$ & $8(1)$ \\
\hline$C(31)$ & $23(1)$ & $31(1)$ & $21(1)$ & $2(1)$ & $9(1)$ & $7(1)$ \\
\hline$C(32)$ & $12(1)$ & $29(1)$ & $15(1)$ & $-1(1)$ & $3(1)$ & $-4(1)$ \\
\hline C(33) & $21(1)$ & $32(1)$ & $24(1)$ & $6(1)$ & $10(1)$ & $2(1)$ \\
\hline$C(34)$ & $23(1)$ & $41(1)$ & $23(1)$ & $6(1)$ & $12(1)$ & $-1(1)$ \\
\hline$C(35)$ & $19(1)$ & $41(1)$ & $21(1)$ & $-6(1)$ & $8(1)$ & $-5(1)$ \\
\hline$C(36)$ & $24(1)$ & $27(1)$ & $27(1)$ & $-5(1)$ & 11(1) & $-2(1)$ \\
\hline $\mathrm{C}(37)$ & 21(1) & $27(1)$ & $20(1)$ & $-1(1)$ & $9(1)$ & $-3(1)$ \\
\hline $\mathrm{C}(38)$ & $28(1)$ & $44(1)$ & $32(1)$ & $-6(1)$ & $17(1)$ & $-4(1)$ \\
\hline$C(16)$ & $27(1)$ & $40(1)$ & $55(1)$ & $19(1)$ & $9(1)$ & $-2(1)$ \\
\hline
\end{tabular}




\subsection{Crystal Structure Determination of 6}

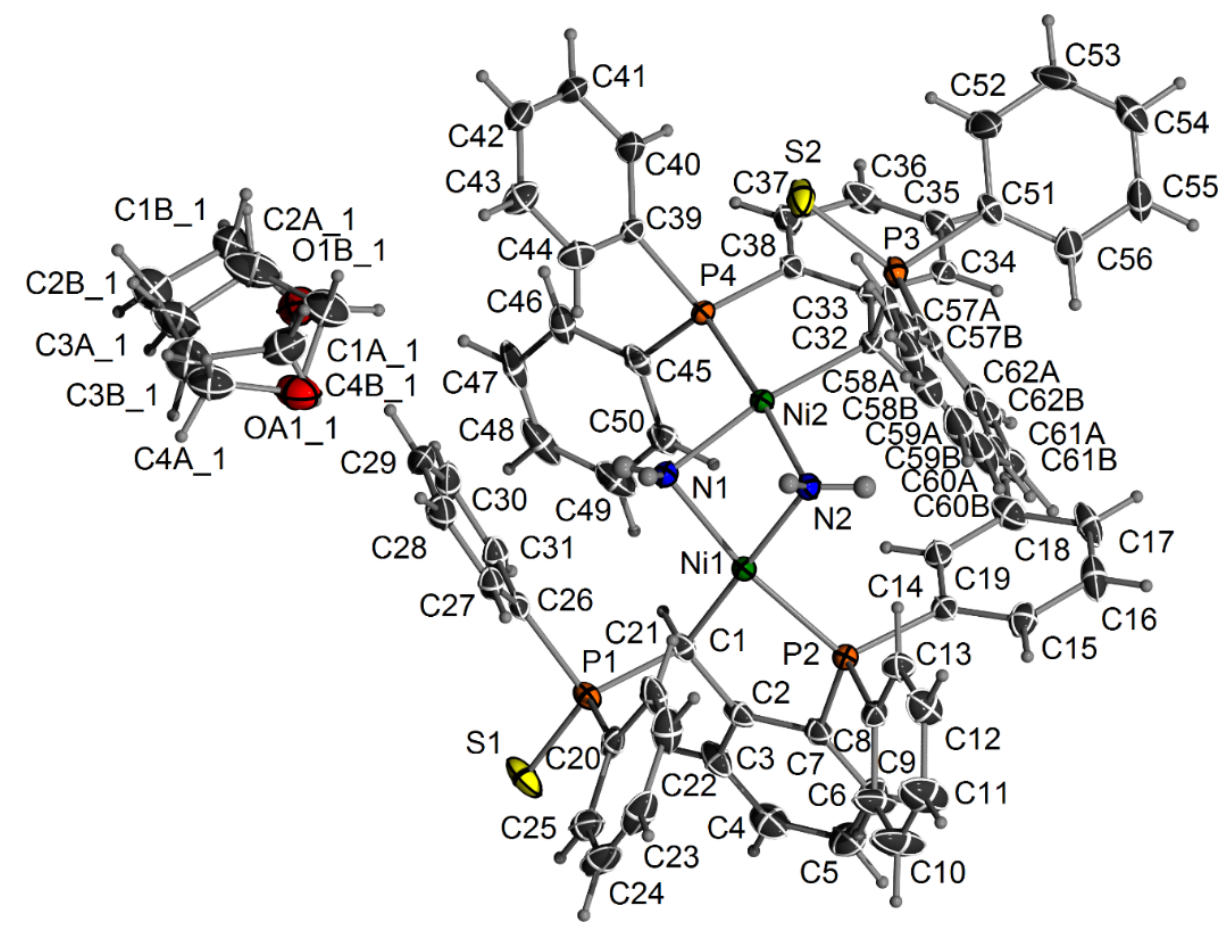

Figure S41. ORTEP Plot of compound $\mathbf{6}_{2}$. Ellipsoids are drawn at the 50\% probability level.

Table S25. Atomic coordinates ( x 10 $)$ and equivalent isotropic displacement parameters $\left(\AA^{2} \times 10^{3}\right)$ for compound 62. $\mathrm{U}(\mathrm{eq})$ is defined as one third of the trace of the orthogonalized $\mathrm{U}_{\mathrm{ij}}$ tensor.

\begin{tabular}{ccccc}
\hline & $\mathrm{x}$ & $\mathrm{y}$ & $\mathrm{z}$ & $\mathrm{U}(\mathrm{eq})$ \\
\hline $\mathrm{Ni}(1)$ & $2876(1)$ & $2478(1)$ & $7379(1)$ & $14(1)$ \\
$\mathrm{S}(1)$ & $106(1)$ & $1930(1)$ & $6577(1)$ & $32(1)$ \\
$\mathrm{P}(1)$ & $1081(1)$ & $2479(1)$ & $6847(1)$ & $17(1)$ \\
$\mathrm{N}(1)$ & $3075(2)$ & $2927(2)$ & $6486(2)$ & $18(1)$ \\
$\mathrm{C}(1)$ & $1925(2)$ & $1871(3)$ & $7089(2)$ & $17(1)$ \\
$\mathrm{Ni}(2)$ & $4116(1)$ & $2613(1)$ & $6720(1)$ & $15(1)$ \\
$\mathrm{P}(2)$ & $2851(1)$ & $1981(1)$ & $8395(1)$ & $16(1)$ \\
$\mathrm{N}(2)$ & $3858(2)$ & $2944(2)$ & $7625(2)$ & $16(1)$ \\
$\mathrm{C}(2)$ & $1759(2)$ & $1221(3)$ & $7599(3)$ & $20(1)$ \\
$\mathrm{C}(3)$ & $1259(3)$ & $595(3)$ & $7415(3)$ & $24(1)$ \\
$\mathrm{P}(3)$ & $5812(1)$ & $2930(1)$ & $7190(1)$ & $18(1)$ \\
$\mathrm{P}(4)$ & $4312(1)$ & $2230(1)$ & $5686(1)$ & $17(1)$ \\
$\mathrm{C}(4)$ & $1115(3)$ & $37(3)$ & $7917(3)$ & $31(1)$ \\
$\mathrm{C}(5)$ & $1465(3)$ & $78(3)$ & $8606(3)$ & $34(1)$ \\
$\mathrm{C}(6)$ & $1978(3)$ & $677(3)$ & $8792(3)$ & $29(1)$ \\
$\mathrm{C}(7)$ & $2119(2)$ & $1241(3)$ & $8288(3)$ & $20(1)$ \\
$\mathrm{C}(8)$ & $2711(3)$ & $2612(3)$ & $9143(2)$ & $21(1)$ \\
$\mathrm{C}(9)$ & $2111(3)$ & $2507(4)$ & $9542(3)$ & $35(1)$ \\
$\mathrm{C}(10)$ & $2033(3)$ & $3006(4)$ & $10112(3)$ & $50(2)$ \\
$\mathrm{C}(11)$ & $2562(3)$ & $3594(4)$ & $10292(3)$ & $47(2)$ \\
$\mathrm{C}(14)$ & $3722(2)$ & $1436(3)$ & $8680(2)$ & $18(1)$ \\
$\mathrm{C}(13)$ & $3230(3)$ & $3220(3)$ & $9315(2)$ & $23(1)$ \\
$\mathrm{C}(12)$ & $3161(3)$ & $3700(3)$ & $9896(3)$ & $31(1)$ \\
$\mathrm{C}(15)$ & $4123(3)$ & $1495(3)$ & $9350(3)$ & $24(1)$ \\
$\mathrm{C}(16)$ & $4793(3)$ & $1079(3)$ & $9514(3)$ & $32(1)$ \\
$\mathrm{C}(17)$ & $5067(3)$ & $594(3)$ & $9018(3)$ & $29(1)$ \\
\hline
\end{tabular}




\begin{tabular}{|c|c|c|c|c|}
\hline $\mathrm{C}(19)$ & 3994(3) & $941(3)$ & $8180(3)$ & $22(1)$ \\
\hline $\mathrm{C}(18)$ & $4662(3)$ & $519(3)$ & $8340(3)$ & $27(1)$ \\
\hline $\mathrm{C}(20)$ & $1605(3)$ & $3682(3)$ & $7802(3)$ & $26(1)$ \\
\hline$C(21)$ & 1511(3) & $4264(3)$ & $8296(3)$ & $34(1)$ \\
\hline$C(22)$ & $806(4)$ & $4359(3)$ & $8546(3)$ & $38(1)$ \\
\hline$C(23)$ & $210(4)$ & $3878(3)$ & $8309(3)$ & $37(1)$ \\
\hline$C(24)$ & 297(3) & $3297(3)$ & $7809(3)$ & $29(1)$ \\
\hline$C(25)$ & $997(3)$ & $3198(3)$ & $7548(2)$ & 20(1) \\
\hline$C(26)$ & $1329(2)$ & $3041(3)$ & $6098(2)$ & $18(1)$ \\
\hline$C(27)$ & $1384(3)$ & $3856(3)$ & $6082(2)$ & 21(1) \\
\hline$C(28)$ & $1599(3)$ & $4240(3)$ & $5492(3)$ & $31(1)$ \\
\hline$C(29)$ & $1783(3)$ & $3804(4)$ & $4920(3)$ & $34(1)$ \\
\hline $\mathrm{C}(30)$ & $1721(3)$ & $3002(3)$ & $4924(3)$ & $33(1)$ \\
\hline $\mathrm{C}(31)$ & $1478(3)$ & 2611(3) & $5502(2)$ & $24(1)$ \\
\hline $\mathrm{C}(32)$ & $5124(2)$ & $2144(3)$ & $7062(2)$ & $15(1)$ \\
\hline $\mathrm{C}(33)$ & $5385(2)$ & $1522(3)$ & $6570(2)$ & $16(1)$ \\
\hline$C(34)$ & 5911(2) & $937(3)$ & $6795(2)$ & $18(1)$ \\
\hline$C(35)$ & 6148(3) & $400(3)$ & $6318(3)$ & $22(1)$ \\
\hline$C(36)$ & $5864(3)$ & $422(3)$ & $5608(3)$ & $28(1)$ \\
\hline$C(37)$ & $5324(3)$ & $986(3)$ & $5376(2)$ & $25(1)$ \\
\hline $\mathrm{C}(38)$ & $5088(2)$ & $1531(3)$ & $5853(2)$ & $18(1)$ \\
\hline$C(39)$ & $4501(3)$ & $2954(3)$ & $5023(2)$ & $22(1)$ \\
\hline $\mathrm{C}(40)$ & $5031(3)$ & $2856(4)$ & $4545(3)$ & $37(1)$ \\
\hline $\mathrm{C}(41)$ & $5127(3)$ & $3434(4)$ & $4045(3)$ & $40(2)$ \\
\hline $\mathrm{C}(42)$ & $4702(3)$ & $4118(3)$ & $4033(3)$ & $32(1)$ \\
\hline $\mathrm{C}(43)$ & $4170(3)$ & $4219(3)$ & $4500(3)$ & $30(1)$ \\
\hline $\mathrm{C}(44)$ & $4066(3)$ & $3635(3)$ & 4989(3) & $27(1)$ \\
\hline $\mathrm{C}(45)$ & $3540(2)$ & $1656(3)$ & $5215(2)$ & 21(1) \\
\hline $\mathrm{C}(46)$ & $3335(3)$ & $1703(3)$ & $4487(3)$ & $27(1)$ \\
\hline $\mathrm{C}(47)$ & $2727(3)$ & $1260(4)$ & $4167(3)$ & $37(1)$ \\
\hline $\mathrm{C}(48)$ & $2331(3)$ & $766(4)$ & $4566(3)$ & $38(1)$ \\
\hline C(49) & $2530(3)$ & $707(3)$ & $5293(3)$ & $32(1)$ \\
\hline $\mathrm{C}(50)$ & $3128(3)$ & $1152(3)$ & $5618(3)$ & $24(1)$ \\
\hline O1A1 & $1667(4)$ & $5666(4)$ & $4362(5)$ & $45(2)$ \\
\hline C1A1 & $2391(6)$ & $5545(8)$ & $4108(7)$ & $44(3)$ \\
\hline $\mathrm{C} 2 \mathrm{~A} 1$ & $2192(10)$ & $5415(12)$ & $3311(9)$ & $51(5)$ \\
\hline C3A1 & $1427(8)$ & $5826(11)$ & $3141(8)$ & $42(3)$ \\
\hline C4A1 & $1259(9)$ & $6162(11)$ & $3851(8)$ & $39(3)$ \\
\hline O1B1 & $2195(8)$ & $5182(8)$ & $3746(8)$ & $40(4)$ \\
\hline C1B1 & $2242(19)$ & $5450(20)$ & $3038(12)$ & $36(5)$ \\
\hline $\mathrm{C} 2 \mathrm{~B} 1$ & $1506(14)$ & $5923(15)$ & $2850(11)$ & $36(5)$ \\
\hline C3B1 & $1273(15)$ & $6160(17)$ & $3568(12)$ & $37(5)$ \\
\hline C4B1 & $1914(14)$ & $5843(11)$ & $4103(10)$ & $42(4)$ \\
\hline $\mathrm{S}(2)$ & $5802(1)$ & $3633(1)$ & $6375(1)$ & $34(1)$ \\
\hline$C(57 \mathrm{~A})$ & $5610(20)$ & $3424(8)$ & $8040(12)$ & $18(3)$ \\
\hline $\mathrm{C}(58 \mathrm{~A})$ & $5452(16)$ & $3005(10)$ & $8639(11)$ & 21(3) \\
\hline$C(59 A)$ & $5285(11)$ & $3400(12)$ & $9242(10)$ & $26(3)$ \\
\hline$C(60 A)$ & $5264(8)$ & $4215(12)$ & $9245(12)$ & $33(4)$ \\
\hline$C(61 A)$ & $5418(10)$ & $4642(8)$ & $8656(13)$ & $29(4)$ \\
\hline$C(62 A)$ & $5600(13)$ & $4240(9)$ & $8063(12)$ & $26(3)$ \\
\hline $\mathrm{C}(51)$ & $6760(2)$ & $2488(3)$ & $7416(2)$ & $20(1)$ \\
\hline $\mathrm{C}(52)$ & $7285(3)$ & $2494(4)$ & $6939(4)$ & $50(2)$ \\
\hline $\mathrm{C}(53)$ & $7987(3)$ & $2120(4)$ & $7089(4)$ & $56(2)$ \\
\hline $\mathrm{C}(54)$ & $8175(3)$ & $1756(3)$ & $7721(3)$ & $32(1)$ \\
\hline$C(55)$ & $7659(3)$ & $1746(4)$ & 8198(3) & $37(1)$ \\
\hline$C(56)$ & $6950(3)$ & $2100(4)$ & $8042(3)$ & $34(1)$ \\
\hline
\end{tabular}




\begin{tabular}{lllll}
\hline $\mathrm{C}(57 \mathrm{~B})$ & $5640(50)$ & $3500(20)$ & $7890(30)$ & $18(3)$ \\
$\mathrm{C}(58 \mathrm{~B})$ & $5460(40)$ & $3170(20)$ & $8520(20)$ & $26(7)$ \\
$\mathrm{C}(59 \mathrm{~B})$ & $5300(30)$ & $3630(30)$ & $9090(20)$ & $32(7)$ \\
$\mathrm{C}(60 \mathrm{~B})$ & $5310(20)$ & $4440(30)$ & $9020(30)$ & $38(9)$ \\
$\mathrm{C}(61 \mathrm{~B})$ & $5540(30)$ & $4790(20)$ & $8420(30)$ & $34(8)$ \\
$\mathrm{C}(62 \mathrm{~B})$ & $5690(30)$ & $4310(20)$ & $7860(20)$ & $23(6)$ \\
\hline
\end{tabular}

Table S26. Anisotropic displacement parameters $\left(\AA^{2} \times 10^{3}\right)$ for compound $\mathbf{6}_{2}$. The anisotropic displacement factor exponent takes the form: $-2 \pi^{2}\left[\mathrm{~h}^{2} \mathrm{a}^{* 2} \mathrm{U}^{11}+\ldots+2 \mathrm{~h} \mathrm{k} \mathrm{a}^{*} \mathrm{~b}^{*} \mathrm{U}^{12}\right]$.

\begin{tabular}{|c|c|c|c|c|c|c|}
\hline & $\mathrm{U}^{11}$ & $\mathrm{U}^{22}$ & $\mathrm{U}^{33}$ & $\mathrm{U}^{23}$ & $\mathrm{U}^{13}$ & $\mathrm{U}^{12}$ \\
\hline $\mathrm{Ni}(1)$ & $12(1)$ & $16(1)$ & $14(1)$ & $0(1)$ & $1(1)$ & $0(1)$ \\
\hline $\mathrm{S}(1)$ & $14(1)$ & $22(1)$ & $58(1)$ & $0(1)$ & $-4(1)$ & $-1(1)$ \\
\hline $\mathrm{P}(1)$ & $13(1)$ & $16(1)$ & $22(1)$ & $0(1)$ & 1(1) & 1(1) \\
\hline $\mathrm{N}(1)$ & $17(2)$ & $24(2)$ & $14(2)$ & $2(2)$ & $1(2)$ & $3(2)$ \\
\hline $\mathrm{C}(1)$ & $15(2)$ & $17(2)$ & $19(2)$ & $-2(2)$ & $2(2)$ & $3(2)$ \\
\hline $\mathrm{Ni}(2)$ & $12(1)$ & $19(1)$ & $13(1)$ & 1(1) & $2(1)$ & 1(1) \\
\hline $\mathrm{P}(2)$ & $14(1)$ & $19(1)$ & $14(1)$ & $2(1)$ & $2(1)$ & 1(1) \\
\hline $\mathrm{N}(2)$ & $14(2)$ & $20(2)$ & $16(2)$ & $0(2)$ & $1(2)$ & $0(2)$ \\
\hline $\mathrm{C}(2)$ & $14(2)$ & $16(2)$ & $29(3)$ & 1(2) & $4(2)$ & $3(2)$ \\
\hline$C(3)$ & $19(2)$ & $18(2)$ & $35(3)$ & $-2(2)$ & $-1(2)$ & $3(2)$ \\
\hline $\mathrm{P}(3)$ & $15(1)$ & $16(1)$ & $21(1)$ & 2(1) & $-3(1)$ & $-1(1)$ \\
\hline $\mathrm{P}(4)$ & $14(1)$ & $26(1)$ & 11(1) & $1(1)$ & $2(1)$ & 2(1) \\
\hline $\mathrm{C}(4)$ & $25(3)$ & $16(2)$ & $50(3)$ & $2(2)$ & $4(2)$ & $-3(2)$ \\
\hline$C(5)$ & $34(3)$ & $24(3)$ & $44(3)$ & $12(2)$ & $7(2)$ & $-2(2)$ \\
\hline$C(6)$ & $28(3)$ & $29(3)$ & $30(3)$ & $13(2)$ & $1(2)$ & $-1(2)$ \\
\hline$C(7)$ & $16(2)$ & $19(2)$ & $26(2)$ & $5(2)$ & $3(2)$ & $2(2)$ \\
\hline $\mathrm{C}(8)$ & $21(2)$ & $28(3)$ & $15(2)$ & $2(2)$ & $2(2)$ & $4(2)$ \\
\hline$C(9)$ & $24(3)$ & $56(4)$ & $26(3)$ & $-13(3)$ & $11(2)$ & $-9(2)$ \\
\hline$C(10)$ & $34(3)$ & $83(5)$ & $36(3)$ & $-28(3)$ & $20(3)$ & $-16(3)$ \\
\hline$C(11)$ & $37(3)$ & $69(5)$ & $36(3)$ & $-27(3)$ & $13(3)$ & $-3(3)$ \\
\hline$C(14)$ & $15(2)$ & $14(2)$ & $25(2)$ & $7(2)$ & $2(2)$ & $-1(2)$ \\
\hline$C(13)$ & $22(2)$ & $25(2)$ & $22(2)$ & $-2(2)$ & $6(2)$ & $3(2)$ \\
\hline$C(12)$ & $26(3)$ & $38(3)$ & $29(3)$ & $-13(2)$ & $1(2)$ & $-1(2)$ \\
\hline$C(15)$ & $27(3)$ & $21(2)$ & $24(2)$ & $3(2)$ & $0(2)$ & $-1(2)$ \\
\hline$C(16)$ & $30(3)$ & $26(3)$ & $36(3)$ & $5(2)$ & $-14(2)$ & $2(2)$ \\
\hline$C(17)$ & $21(2)$ & $17(2)$ & $46(3)$ & $2(2)$ & $-10(2)$ & $5(2)$ \\
\hline$C(19)$ & $19(2)$ & $24(2)$ & $24(2)$ & $5(2)$ & $0(2)$ & $-1(2)$ \\
\hline$C(18)$ & $23(2)$ & $20(2)$ & $40(3)$ & $-1(2)$ & $5(2)$ & $1(2)$ \\
\hline$C(20)$ & $23(2)$ & $28(3)$ & $27(3)$ & $-5(2)$ & $-2(2)$ & $12(2)$ \\
\hline$C(21)$ & $41(3)$ & $32(3)$ & $27(3)$ & $-8(2)$ & $-6(2)$ & $13(2)$ \\
\hline$C(22)$ & $62(4)$ & $36(3)$ & $18(3)$ & $-2(2)$ & $11(2)$ & $24(3)$ \\
\hline$C(23)$ & $48(3)$ & $39(3)$ & $30(3)$ & $8(2)$ & $23(3)$ & $20(3)$ \\
\hline$C(24)$ & $30(3)$ & $28(3)$ & $30(3)$ & $6(2)$ & $12(2)$ & $6(2)$ \\
\hline$C(25)$ & $25(2)$ & $18(2)$ & $16(2)$ & $3(2)$ & $4(2)$ & $10(2)$ \\
\hline$C(26)$ & $12(2)$ & $25(2)$ & $16(2)$ & $0(2)$ & $-4(2)$ & $3(2)$ \\
\hline$C(27)$ & $19(2)$ & $20(2)$ & $22(2)$ & 1(2) & $-1(2)$ & $5(2)$ \\
\hline$C(28)$ & $25(3)$ & $31(3)$ & $36(3)$ & $10(2)$ & $-2(2)$ & $2(2)$ \\
\hline$C(29)$ & $26(3)$ & $55(4)$ & $22(3)$ & $16(2)$ & $7(2)$ & $13(2)$ \\
\hline$C(30)$ & $32(3)$ & $48(3)$ & $18(2)$ & $-1(2)$ & $-3(2)$ & 19(3) \\
\hline $\mathrm{C}(31)$ & $25(2)$ & $28(3)$ & $18(2)$ & $-6(2)$ & $-3(2)$ & $9(2)$ \\
\hline$C(32)$ & $15(2)$ & $15(2)$ & $14(2)$ & $3(2)$ & $1(2)$ & $-1(2)$ \\
\hline$C(33)$ & $14(2)$ & $20(2)$ & $14(2)$ & $-1(2)$ & $4(2)$ & $-4(2)$ \\
\hline$C(34)$ & $16(2)$ & $19(2)$ & $19(2)$ & $1(2)$ & $2(2)$ & $-3(2)$ \\
\hline$C(35)$ & $16(2)$ & $22(2)$ & $29(3)$ & $-3(2)$ & $3(2)$ & $3(2)$ \\
\hline
\end{tabular}




\begin{tabular}{|c|c|c|c|c|c|c|}
\hline$C(36)$ & $20(2)$ & $35(3)$ & $29(3)$ & $-16(2)$ & $5(2)$ & $4(2)$ \\
\hline $\mathrm{C}(37)$ & $17(2)$ & $40(3)$ & $17(2)$ & $-5(2)$ & $0(2)$ & $5(2)$ \\
\hline $\mathrm{C}(38)$ & $12(2)$ & $25(2)$ & $16(2)$ & $-1(2)$ & 1(2) & $-1(2)$ \\
\hline C(39) & $17(2)$ & $36(3)$ & $12(2)$ & $1(2)$ & $2(2)$ & $0(2)$ \\
\hline $\mathrm{C}(40)$ & $26(3)$ & $57(4)$ & $30(3)$ & $20(3)$ & $8(2)$ & $15(3)$ \\
\hline $\mathrm{C}(41)$ & $26(3)$ & $68(4)$ & $27(3)$ & $25(3)$ & $11(2)$ & $9(3)$ \\
\hline $\mathrm{C}(42)$ & $27(3)$ & $45(3)$ & $25(3)$ & $16(2)$ & 1(2) & $-4(2)$ \\
\hline $\mathrm{C}(43)$ & $38(3)$ & $24(3)$ & $29(3)$ & $5(2)$ & $7(2)$ & $-1(2)$ \\
\hline $\mathrm{C}(44)$ & $30(3)$ & $28(3)$ & $24(3)$ & $-3(2)$ & $9(2)$ & $-1(2)$ \\
\hline $\mathrm{C}(45)$ & $15(2)$ & $25(2)$ & $23(2)$ & $-7(2)$ & $1(2)$ & $8(2)$ \\
\hline$C(46)$ & $25(2)$ & $32(3)$ & $22(2)$ & $-8(2)$ & $-5(2)$ & $12(2)$ \\
\hline$C(47)$ & $26(3)$ & $51(4)$ & $28(3)$ & $-20(3)$ & $-15(2)$ & $17(3)$ \\
\hline $\mathrm{C}(48)$ & $17(2)$ & $41(3)$ & $53(4)$ & $-23(3)$ & $-9(2)$ & $8(2)$ \\
\hline $\mathrm{C}(49)$ & $19(2)$ & $34(3)$ & $44(3)$ & $-12(2)$ & $3(2)$ & $-1(2)$ \\
\hline $\mathrm{C}(50)$ & $19(2)$ & $31(3)$ & $23(2)$ & $-8(2)$ & $2(2)$ & $2(2)$ \\
\hline O1A1 & $37(4)$ & $38(4)$ & $63(6)$ & $14(4)$ & $19(4)$ & $3(3)$ \\
\hline C1A1 & $34(6)$ & $45(7)$ & $54(8)$ & $-4(6)$ & $6(5)$ & $-8(5)$ \\
\hline $\mathrm{C} 2 \mathrm{~A} 1$ & $37(7)$ & $47(9)$ & $72(15)$ & $-15(11)$ & $18(11)$ & $-13(6)$ \\
\hline C3A1 & $31(6)$ & $49(8)$ & $46(9)$ & $-21(7)$ & $6(6)$ & $-10(5)$ \\
\hline C4A1 & $39(6)$ & $32(6)$ & $48(9)$ & $-6(7)$ & $15(7)$ & $-1(4)$ \\
\hline O1B1 & $42(7)$ & $39(8)$ & $40(8)$ & $2(6)$ & $11(6)$ & $1(6)$ \\
\hline C1B1 & $28(9)$ & $45(11)$ & $36(10)$ & $2(9)$ & $6(9)$ & $1(7)$ \\
\hline $\mathrm{C} 2 \mathrm{~B} 1$ & $34(9)$ & $28(9)$ & $44(11)$ & $-12(9)$ & $1(8)$ & $0(7)$ \\
\hline C3B1 & $46(10)$ & $20(8)$ & $46(13)$ & $-10(11)$ & $8(10)$ & $2(7)$ \\
\hline C4B1 & $52(12)$ & $37(9)$ & $40(9)$ & $4(7)$ & $17(8)$ & $2(8)$ \\
\hline $\mathrm{S}(2)$ & $26(1)$ & $33(1)$ & $40(1)$ & 21(1) & $-8(1)$ & $-9(1)$ \\
\hline$C(57 \mathrm{~A})$ & 11(3) & $22(4)$ & $19(8)$ & $-2(4)$ & $-8(8)$ & $4(4)$ \\
\hline $\mathrm{C}(58 \mathrm{~A})$ & $13(5)$ & $27(5)$ & $20(6)$ & $-2(4)$ & $-4(5)$ & $5(5)$ \\
\hline $\mathrm{C}(59 \mathrm{~A})$ & $19(5)$ & $37(7)$ & $18(6)$ & $-6(5)$ & $-5(4)$ & $8(6)$ \\
\hline$C(60 A)$ & $22(5)$ & $44(9)$ & $31(7)$ & $-18(6)$ & $-11(5)$ & $16(5)$ \\
\hline$C(61 A)$ & $24(8)$ & $19(6)$ & $41(12)$ & $-7(6)$ & $-8(9)$ & $4(5)$ \\
\hline$C(62 A)$ & $24(6)$ & $20(5)$ & $31(8)$ & $-3(5)$ & $-6(6)$ & $4(4)$ \\
\hline $\mathrm{C}(51)$ & $14(2)$ & $19(2)$ & $26(2)$ & $-1(2)$ & $-2(2)$ & $0(2)$ \\
\hline $\mathrm{C}(52)$ & $30(3)$ & $65(4)$ & $57(4)$ & $40(3)$ & $18(3)$ & 13(3) \\
\hline $\mathrm{C}(53)$ & $25(3)$ & $73(5)$ & $75(5)$ & $37(4)$ & $29(3)$ & $14(3)$ \\
\hline $\mathrm{C}(54)$ & $17(2)$ & $29(3)$ & $49(3)$ & $-1(2)$ & $-3(2)$ & $2(2)$ \\
\hline $\mathrm{C}(55)$ & $36(3)$ & $52(4)$ & $21(3)$ & $-3(2)$ & $-7(2)$ & $23(3)$ \\
\hline $\mathrm{C}(56)$ & $31(3)$ & $52(4)$ & $18(2)$ & $-5(2)$ & $0(2)$ & 19(3) \\
\hline $\mathrm{C}(57 \mathrm{~B})$ & $11(3)$ & $22(4)$ & $19(8)$ & $-2(4)$ & $-8(8)$ & 4(4) \\
\hline $\mathrm{C}(58 \mathrm{~B})$ & $17(13)$ & $36(13)$ & $22(11)$ & $0(8)$ & $-6(10)$ & $6(13)$ \\
\hline $\mathrm{C}(59 \mathrm{~B})$ & $16(11)$ & $53(17)$ & $26(12)$ & $-2(11)$ & $0(10)$ & $17(13)$ \\
\hline $\mathrm{C}(60 \mathrm{~B})$ & $27(13)$ & $50(16)$ & $35(19)$ & $-8(12)$ & $-5(16)$ & $15(13)$ \\
\hline $\mathrm{C}(61 \mathrm{~B})$ & 27(17) & $36(12)$ & $37(17)$ & $-9(11)$ & $-9(11)$ & $17(13)$ \\
\hline $\mathrm{C}(62 \mathrm{~B})$ & 19(14) & $19(7)$ & $26(15)$ & $-4(8)$ & $-14(11)$ & $3(7)$ \\
\hline
\end{tabular}




\section{Computational Studies}

Cartesian coordinates of complex 1-Ni

$\mathrm{E}=-4244.46424027 \mathrm{E}_{\mathrm{H}}$

Ni -0.398981 $1.047795-0.712279$

S $1.4741441 .441497-1.990897$

P $2.2003030 .525324-0.309144$

C 0.8475570 .7086480 .696306

C 3.6662002 .3109731 .200265

H 2.6722782 .6373061 .522294

C 3.7612321 .2480660 .295625

C 0.5310680 .1946741 .991464

P - $1.660088-0.0468690 .613142$

C 4.8226832 .9229731 .677098

H 4.7489663 .7489292 .388735

C $1.404804-0.0218713 .088869$

H 2.4747580 .1730712 .974245

C 6.0738972 .4801361 .246557

H 6.9812762 .9602311 .621555

C $0.904841-0.4336324 .316549$

H $1.599686-0.5686495 .150708$

C $5.0158140 .804421-0.134282$

H $5.087630-0.031667-0.834929$

C $-1.339706-0.5163523 .447958$

H - $2.407757-0.7229903 .569448$

C $2.648201-1.196940-0.736386$

C $-0.843570-0.0993452 .217149$

C $2.720837-1.640407-2.060396$

H $2.561748-0.919557-2.866745$

C - $1.650578-1.7877380 .051223$

C $2.966772-2.985786-2.335129$

H $3.027239-3.325283-3.372477$

C $-0.811639-2.140229-1.011542$

H $-0.236388-1.361105-1.519923$

C $3.133381-3.894645-1.291348$

H $3.321129-4.949340-1.507412$

C $-0.683844-3.474873-1.392641$

$\mathrm{H}-0.006273-3.738616-2.207507$

C $-1.403109-4.462046-0.723402$

H - $1.303251-5.508855-1.020782$

C -3.4104360 .3632200 .910329$

C $-4.465941-0.2711210 .244430$

H - $4.265245-1.130695-0.399746$

C -5.7750370 .1811090 .406837$

H -6.590955 -0.322655 -0.117012

C -6.0425591 .2681831 .237047$

H - 7.0685471 .6217091 .363603

C -4.996394 1.8996591 .911745

H -5.201389 2.7482152 .568778

C $-2.362651-2.7861410 .728613$

H -3.002025 -2.523042 1.575295

C $-2.244867-4.1160650 .335953$

H - $2.802205-4.8906400 .868339$

C -3.6883351 .4526671 .748818$

H - 2.8672131 .9504132 .272301

C $3.054289-3.4564700 .031677$

H $3.171758-4.1676280 .852726$

C $-2.6714790 .211150-3.112082$

H -3.351888 0.561915 -3.903244 
H $-3.239566-0.372938-2.375142$ H - $1.909279-0.449188-3.550353$ C $2.815022-2.1142050 .310812$ H $2.731479-1.7817301 .348728$ C -3.227537 2.680979-1.765112 H $-2.8228673 .604089-1.325094$ H -3.831363 2.174377 -0.999786 H -3.866749 2.938681 -2.623626 P $-1.8339511 .607506-2.273892$ C - $1.1281732 .535013-3.682587$ $\mathrm{H}-0.3464291 .923671-4.156417$ H -0.651955 $3.452589-3.308176$ H -1.899942 2.792563 -4.424043 C 6.1701751 .4230170 .341089 H 7.1511091 .0754820 .007750 C $-0.463343-0.6714604 .520932$ H -0.832033 -0.983651 5.499842

Cartesian coordinates of complex 1-Pd $\mathrm{E}=-3437.85533678 \mathrm{E}_{\mathrm{H}}$ $\mathrm{Pd}-0.1218970 .1511090 .258911$ S -1.610806 2.0391780.602205 P $0.581796-1.970127-0.157232$ P -2.972251 0.5664440 .143597 P 1.8438831 .4424320 .288255 C $-1.920771-0.7621590 .046090$ C $-2.084806-2.103871-0.384663$ C $-3.322348-2.758609-0.644072$ H $-4.254814-2.198008-0.546676$ C -3.359112 -4.094571-1.005253 H $-4.329049-4.562979-1.196315$ C -2.189049 -4.863488 -1.124864 H -2.240991 -5.917977 -1.401594 C $-0.963351-4.251805-0.882395$ H $-0.035303-4.826434-0.963180$ C $-0.913330-2.899011-0.545268$ C $-3.8762961 .005550-1.381885$ C $-5.1216950 .432439-1.668610$ H -5.623956 -0.189186 -0.922635 C $-5.7326520 .666276-2.899421$ H $-6.7046610 .216772-3.116634$ C -5.107916 1.474807 -3.847445 H -5.590331 $1.660741-4.810193$ C -3.868721 2.051302 -3.563031 H $-3.3812302 .692951-4.301216$ C -3.252583 1.816474 -2.337499 H -2.286016 2.267354 -2.095345 C -4.282144 0.448572 1.404691 C -5.2596141 .4408721 .541256$ H -5.2746162 .2910790 .853600$ C -6.211108 1.341199 2.552267 H -6.9729442 .1168402 .662245$ C -6.192705 0.2502813.423934 H -6.943857 0.172356 4.213946 C -5.219130 -0.738285 3.288624 H -5.206387 -1.592331 3.970089 C $-4.258626-0.6377012 .283053$ H -3.477511 -1.394337 2.161792 C $1.298169-2.8367151 .284466$ C $0.741156-2.5403222 .537269$ H $-0.047777-1.7843162 .602535$ C $1.202398-3.1877093 .678722$ 
H $0.766735-2.9475064 .651363$ C $2.225227-4.1334513 .582170$ H $2.592888-4.6357094 .480265$ C $2.774835-4.4373412 .338114$ H $3.570224-5.1822922 .257480$ C $2.311256-3.7958231 .189156$ H $2.745143-4.0395610 .216857$ C $1.766336-2.206530-1.518877$ C $1.314785-2.609376-2.780559$ H $0.259020-2.852189-2.921817$ C $2.208297-2.701897-3.846731$ H $1.846604-3.019687-4.827590$ C $3.554454-2.394364-3.662891$ H $4.253201-2.467937-4.499639$ C $4.007703-1.981561-2.409763$ H $5.057957-1.720979-2.263004$ C $3.118672-1.878974-1.345185$ H $3.481270-1.533652-0.373834$ C 3.1755841 .1804561 .518380 C $3.330357-0.0889812 .083892$ H $2.630145-0.8846731 .828857$ C $4.356807-0.3397292 .992802$ H $4.457516-1.3376873 .425933$ C 5.2320960 .6828543 .353352 H 6.0337720 .4914844 .070823 C 5.0739981 .9574382 .807375 H 5.7505522 .7650893 .097248 C 4.0504542 .2087361 .896435 H 3.9218343 .2119771 .482605 C 1.4978563 .2286730 .478128 C 0.9421273 .6588181 .691397 H 0.7455332 .9353572 .487145 C 0.6290275 .0006451 .881655 H 0.1940845 .3250942 .829715 C 0.8538905 .9238760 .859902 H 0.6001976 .9763011 .007613 C $1.3947865 .498971-0.351244$ H $1.5686096 .216930-1.156383$ C $1.7176254 .155682-0.544158$ H $2.1428593 .828518-1.495300$ C $2.6980391 .352465-1.328500$ C $1.9133961 .055004-2.450344$ H $0.8461890 .851231-2.315752$ C $2.4910550 .997646-3.715438$ H $1.8727840 .753873-4.582055$ C $3.8573511 .229562-3.869368$ H $4.3127931 .174702-4.861138$ C $4.6444081 .518469-2.755375$ H $5.7166951 .693678-2.872397$ C $4.0687381 .580044-1.487719$ H $4.6920031 .797001-0.617380$

[1] a) G. M. Sheldrick, Acta Cryst. 2008, A64, 112; b) A. Thorn, B. Dittrich, G. M. Sheldrick, Acta Cryst. 2012, A68, 448; c) G. M. Sheldrick, Acta Cryst. 2008, A64, 112; d) G. M. Sheldrick, Acta Cryst. 2015, C71, 3. 